\section{\% LA-6116-MS \\ Informal Report}

\section{(ENDF-223)}

UC-34c and UC-80

Reporting Date: September 1975 Issued: October 1975

\title{
ENDF/B-IV Fission-Product Files: Summary of Major Nuclide Data
}

\author{
by
}

T. R. England

R. E. Schenter*

- Visiting Staff Member. Hanford Engineering Development Labcratory.

Richland, WA 99352. 
In the interest of prompt distribution, this report was not edited by the Technical Information staff.

Work supported by the U.S. Energy Research and Development Administration, Division of Reactor Research and Development, and the Nuclear Regulatory Commission, Office of Standards Develnpment.

Printed in the United Stutes of America. Available trom

National Technical information Service

U S Department of Commerce

5285 Port Roral Rood

Springtiold, VA 2015!

Price: Printed Copy \$1.00 Mieroficte $\$ 2.25$

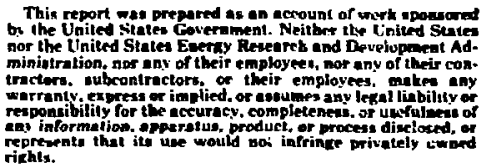

richts. 


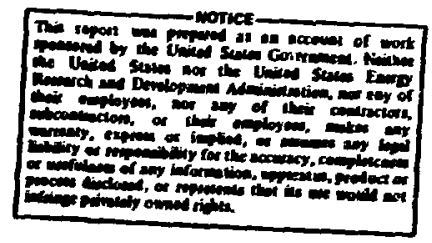

ENDF/B-IV FISSION-PRODUCT FILES: SLAMARY OF MAJOR NUCLIDE DATA

by

T. R. England and R. E. Schenter

\section{ABSTRACT}

The major fission-product parameters $\left[\sigma_{t h}, R I, \tau_{1 / 2}, \bar{E}_{B}, \bar{E}_{\gamma}, \bar{E}_{q}\right.$, decay and (n,Y) branching, $Q$, and AWR] abstracted from ENDF/B-IV files for 824 auclides are sumarized. These data are most of ten requested by users concerned with reactor design, reactor safety, dose, and other sundry studies. The few known file errors are corrected to date. Tabular data are listed by increasing mass number.

\section{INTRODUCTION}

Two and o'e-half years ago, a large task force was organized to expand the ENDF/B fission-product data from a 55-nuclide cross-section data set to a comprehensive file which, at present, encompasses data on 824 nuclides; these data include cross sectlons, decay parameters, and yields. Approximately 30 people from various industrial and government laboratories have cooperated in this task. This ad hoc group of people was divided Into several subcommitrees working under the Cross Section Evaluation Working Group (CSEWG) Fission-Product Subcomittee Task Force to produce evaluated data for use in decay heat and burnup calculations as part of the general effort to produce an Evaluated Nuclear Data File (ENDF/B).

The motivation for an expanded file began witi the need for a reference set of fission-product decay data for calculating decay heating during "lossof-coolant accidents" (I.OCA). however, the task force meabers recognized the need for expanded fission-product microscopic cross-section data, adequate for thermal and fast reactor analysis; improved fission yieids; and detalled fission-product gamma "line" data for a number of applications, including absorption buildup, waste disposal and fuel namagement, shielding (spectra), fuel integrity (gas content), and the bulldup of radiologically hazardous and toxic products.
A complete listing of the decay and cross-section files requires several thousand pages. In this report, we have listed the parameters of interest to many users in a compact, readable formac zequiring less than 39 pages. The forwat of this listing was originally designed as a quick reference to cecay data in early versions of the file (457) for use in data tesing and as an aid in forming the nuclide chains. However, this data listing has proven to be a useful reference for the occasional user unfaniliar with ENDF/B formats and for particular applications requiring subsets of these data, such as in dose studies. A more extensive, detailed data listing will likely be issued by Brookhaven National Laboratory (BNL) as part of the ENDF/B-IV documentation, organized for biomedical and reactor uses.

We have included thermal cross-section and resonance integral data derived fron the ENDF/B-IV files and thermal capture branching data which are essential to the use of these cross sections but which are not in ENDF/B-IV. We have also included a list of internal conversion electron energies (as fractions of the transition energy), a comparison of average energles and $Q$ valuts (calculated from the decay spectra) with the tabulated values in ENDF/B-iv, and a list of known file errors found to date. The yield data and decay spectra are not ticluded in this report.

The sumary data in Table VII form the core of this report. 
II. ENDE/B-IV CONTENT

The ENDF/B-IV fission-product files contain approprlate data for 824 nuclides. Of these, 181 have cross-section evaluations (capture, elastic, inelastic, and total from $10^{-5} \mathrm{eV}$ to $20 \mathrm{MeV}$ ). Thirty-six nuclides have other cross-section evaluations such as $(n, 2 n),(n, t),\left(n, n^{\prime} p\right),(n, d)$, etc. The radiative capture cross sections were identified as being suificiently comprehensive for detailed estimates of absorption buildup in any contemporary reactor. One hundred eighty nuclides have experimental data on $\mathrm{E}^{-}$ end-point energies and $\gamma$ "IIne" data (energies and intensities). 1 sume of these nuclides have several hundred resolved gama lines, but the average number per nuclide is 31 gamma energies and 9 beta end-point energies. All radioactive nuclides (711) have evaluated data for the average $B^{-}$energy $\left(\bar{E}_{\beta}\right)$, total $Y$ energy $\left(\bar{E}_{\gamma}\right)$, half-lives, branching, and other data. These Gata should be adequate for sumation calculations of decay heat and, probably, the $\gamma$ spectral shape. $^{2-4}$ These tilles contain approximately 300000 dita entries. In addition, there are ten sets of direct fission yields for six fissionable nuclides for one or wore neutron fission encrgies ( 12000 entries). Each set contains wore than 1100 yields and appears with the cross sections of the fissionable nuclides In the "General Purpose File."

Tables I and II summarize the number of nuclides having data of various types; the summary of fission yield types in Table II is included for completeness.

It should be noted that isomeric states, but not ground states, in ENDF/B-IV files do not include states having half-ilves $<0.1 \mathrm{~s}$.

Table VII contains the sumary of fission product parameters ( $\sigma^{\prime} s, \tau_{1 / 2}, \bar{E}_{\beta}, \bar{E}_{\gamma}$, branching fractions, etc.) in the format described in the following section. In this table, the nuclides are grouped by mass number, $A$, beginning with the smallest value, and by increasing $z$ value for a given value of A. (This is not the same as the "MaT" ordering as given on the ENDF/B-IV files which are ordered on $Z$ then $A_{.}$) In the table, any isomeric states follow the ground state for a given $z, A$.

In these tables, we have included the resonance Integrals ( $E_{\text {cut }}=0.5 \mathrm{eV}$ and $\mathrm{T}=0^{\circ} \mathrm{K}$ ) and the thermal radiative capture cross sections at $0.0253 \mathrm{eV}$ for the 181 nuclides having cross-section data.
These values appear in (File 1) comments in ENDF/BIV. For four nuclides, ${ }^{87} \mathrm{Sr},{ }^{113} \mathrm{In},{ }^{115} \mathrm{In}$, and ${ }^{123} \mathrm{Te}$, we have used subsequent, more accurate calculations of their resonance integrals. In most cases, the resonance parameters are takan from the BliL report BNL-325 (June 1973) with adjustments in background, consistent with experimental uncertainties, to give experimental thermal values and resonance integrals ( 10 ). For most users (thermal reactors), these are the cross sections most of ten requested; these values are suffictent for determining the importance of $(n, \gamma)$ coupling in forming ruclide chains. In addition to the thermal and resonance integral cross sections, branching cross sections are given. These are needed for those cases where the $(n, \gamma)$ reaction could create an isoneric state. These data are not Included in ENDF/B-IV. The fractional amounts of thermal and resonance capture leading to Isomeric states are essentially Identical. Therefore, we have Incorpurated a preliminary compilation of branching cross sections into Table VII in the form of $(n, \gamma)$ branching fractions where the branching could produce Isomeric states.

The remaining data in Table VII were processed. directly from the six ENUF/B-IV tapes except for correctlons noted in Sec. $V$ of this report. Corrected data are flagged in Table VII to indicate differences from ENDF/B-IV values.

The total gana energies listed in the EMDE/BIV files (and Table VII) are actually transition energies and therefore Include any internal conversion energy. This is discussed in Sec. VI.

\section{FORMAT OF DATA INCLUDED IN TABLE VII}

The colum headings of Table VII apply to the first line of data for each nuclide; if there is more than one decay wode, or if capture cross sections are in the flies, two or more data Ifnes are used. The listed data per line is:

Line 1:

Symbol - charge, chemical symbol, mass, and state Identifier (blank for ground state, $H_{0} N$ for first and second iscmeric stazes).

ZZAMAS - nuweric ID $=10000 * Z+10 * A+5$, where $S=$ state $(0,1,2, \ldots$ for ground, first, and second Isomaric atates).

Half-Itfe - decay half-11fe in seconds. 
E-Beta - average beta energy per decay in eV.

E-Ganma - total gamma energy per decay in eV. (Includes internal conversion energy; see Sec. VI.)

E-Alphe - average alpha energy per decay in ev. (Includes recoil.)

RTYP - type of decay (def Ined below).

$\underline{R E S}$ - state of daughter (0.0 for ground state, 1.0 for first isoneric state, etc.)

$Q-Q$ value in $e v$ for the decay mode.

Brenchlng - branching fraction for decay mode.

AWE - atomic: weight ratio.

NDK - numbe; of decay modes.

NSP - number of types of spectra.

MAT - matertal number running from 1 to 825 (MAT 251 removed from files).

Iine $2, \ldots$ :

If there is more than one decay mode (NDK > 1) the RTYP, RFS, Q-value, and Branching are ilsted (one line per mode).

Last line:

$(n, \gamma)$ cross section $\left[\sigma_{t h}=\sigma(E=0.0253 \mathrm{eV})\right]$. resonance lntegral, and $(n, Y)$ "branchlngs" are 1isted. (One hundred eighty-one of the nuclides have cross sections in various detall. For these, the cross sections at $0.0253 \mathrm{eV}$ and resonance integrals are listed under the E-Beta and E-Gamma colums. In addition, the suggested $(n, \gamma)$ branchings to the ground, first, and second isomerle states are 11sted, as described in Sec. II.)

The RTYP decay mode identifier has the following meanings:

RTYP Mode of Decay

I. $0 \mathrm{~B}^{-}$

$2.0 B^{+}$or $E C$

3.0 Isomeric transition

$4.0 \quad \alpha$

5.0 Delayed neutron $\left(\beta^{-}, n\right)$

6.0 Spontaneous fission

IV. COMPARISON OF CALCULATED ENERGIES USING ENDF/BIV SPECTRAL DATA WITH ENDF/B-IV TABULATED VALUES ENDF/B-IV files contain beta end-point energies

$\left(E_{B_{i}}\right)$ and relative intensities $\left(I_{B_{1}}\right)$, gamma energies $\left(E_{\gamma_{1}}^{B_{i}}\right)$ and relative intensitities $\left(I_{\gamma_{1}}\right)$, and other, IInfted spectral data for 180 of the 711 radioactive nuclides. 1 Each such spectra also coztains a normallzation factor (F). The average beta and total gamms energies tabulated in the files for these $180 \mathrm{nu}-$ clides are calculated from Eqs. (1)-(3):

$$
\bar{E}_{Y_{c}}=\frac{F_{Y}}{100} \sum_{1} E_{Y_{1}} I_{Y_{1}}
$$

$$
\bar{E}_{B_{c}}=\frac{F_{B}}{100} \sum_{1} E_{B_{1}} I_{B_{1}} f_{1}\left(E_{B_{1}}\right)
$$

$$
f_{1}\left(E_{B_{1}}\right)=\frac{1}{4} \frac{2 w_{0}^{2}+8 w_{0}+10}{w_{0}^{2}+5 w_{0}+10}
$$

where

$$
W_{0} \equiv \frac{E_{B_{1}}}{0.511 \times 10^{6}}
$$

Is the beta end-point energy in $m_{0} c^{2}$ units and $f_{i}\left(E_{B_{1}}\right)$ is the approximate ratio of the average beta energy to the beta end-point energy, as derived In Ref. 5 and later reduced to the stopier form of Eq. (3). As nuted In Ref. 1, the simple form of Eq. (3) agrees with an exact averaging for allowed and first forbidden energies within a few percent (<3) for the fisston-product nuclides and decay energies of interest here. (For a few cases of first forbidden, unique transitions, the $f_{i}$ values obtained from Ref. 6 were used.)

In a few cases (38), the files contain internal conversion coefficlents $\left(C_{Y_{f}}\right)$ which can be used to calculate the Internal conversion energy in Eq. (4)

$$
\bar{E}_{I c c}=\frac{F_{Y}}{100} \sum E_{\gamma_{I}} I_{\gamma_{i}} c_{\gamma_{i}}
$$

Fon EIDF/B-IV, the listed value for the total goma energy, $\overline{\mathrm{E}}_{\gamma}$, includes $\overline{\mathrm{E}}_{\text {Ice }}$.

As a partial check on the final ENDF/B-IV files, we have computed the averages of $\bar{E}_{\beta_{c}}$ and $\overline{\mathrm{E}}_{\gamma_{c}}$ (and the $\bar{E}_{\text {Icc }}$ component) along with the average neutrino energy [obtained by replacing $f_{i}$ with $\left(1-f_{i}\right)$ in Eq. (2)]. In addition, the $Q$ value was calculated using Eq. (5)

$$
Q_{c}=\bar{E}_{\beta_{c}}+\bar{E}_{\gamma_{c}}+\bar{E}_{v_{c}}
$$


The spectral data in EMDFib-IV are not separated on the basis of the decay wodes; therefore, Eq. (5) Is the total energy per decay. If there was more chan one decay mode, the values frow Eq. (5) were compared with $Q$ values weighted by the decay branching fractions. The $Q$ values in ENDF/B-IV were generally obtained from mass lav compilations, ${ }^{7,8}$ not the spectra data, and a comparison with the calculated values serves as a check on the self-consistency and posstble source of error in the $Q$ values or spectral data.

The comparisons of $\bar{E}_{B}$ and $\bar{E}_{\gamma}$ show that the calculations differ from ENDF/B-IV values by $>0.01 \%$ in only 23 cases, and by $>1 \%$ for 10 nuclides. For

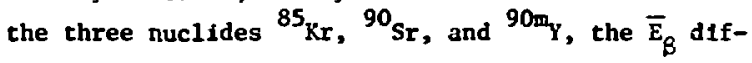
erences are $-10.1,-12.9$, and $-10.6 \%$, respectively; these three nuclides required first forbidden unique shape corrections to the calculated $\overline{\mathbf{E}}_{\beta}$. Except for ${ }^{104 m_{\mathrm{Rh}}}$, only these three nuclides show differences exceeding $5 \%$. The ${ }^{104 \mathrm{~m}_{\mathrm{Rh}}}$ nuclide is a special case; the normalization factor given in ENDF/B-IV is zero and there are typozraphical errors in the garma incensity data. In order to force agreement with the total gama energy, after correcting the typographical errors in the spectra, the value of $\mathbf{F}$ should be 0.018535 .

In Table III, the percent differences of the calculated $\bar{E}_{\beta}, \vec{E}_{Y_{c}}$, and $Q_{c}$ from the ENDF/B-IV values are listed for 69 nuclides; of the 180 nuclides having spectral data, only these differed by more than $1 \%$ in one or more of the three calculated energies; the majority are due to $Q$ differences.

The calculated $Q_{c}$ values using Eq. (S) are compared with ENDF/B-IV Q values (weighted by branching fractions) In Table IV.

Thirty-one of the 180 nuclides having spectral data have calculated $Q_{c}$ values which differ from the tabulated vales by $\geqslant 3 \%$. (Approxtmately 158 of the 180 nuclides have tabulated uncertainties in the ENDF/B-IV files; for 63 of these, the calculated $Q$ is outside the ENDF/B-IV uncertainty. These are identffied with an asterisk ( $($ ) in Table IV.) The $Q$ difference exceeds $5 \%$ in only 12 cases and $10 \%$ for

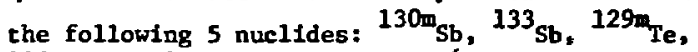
${ }^{136} \mathrm{Cs}$, and $152 \mathrm{mPE}$. Except for ${ }^{136} \mathrm{Cs}$, these have been corrected for this report (Table VII) as noted

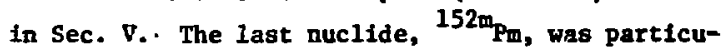
larly discrepant ( 34\%). The beta spectra for this nuclide is not vell known and the files 1 lst only the rost. - iricant cransic ions. The $\bar{E}_{E}$ should be $0.9 \mathrm{Nel}$. nr larger, razher chan $-0.4 \mathrm{MeV}$.

\section{v. FILE ERRORS}

Some corrections have already been made in the first issue of ENDF/B-IV. Comparisops of decay energles and $Q$ values, as in the previlous section, resulced in an additional 13 nuclides still requiring file corrections. These are listed in Table $v$ along with corrections.

Twelve of the thirteen correcticns are incorporated into the data sumary in Table VII. Table VII values differing from ENDF/B-IV are flagged.

\section{INTERHAL CONVERSION ENERCIES}

The ENDF/B-IV fission-product data were corptled for use in calculating total decay heating and absorption. Neutron cross sections, fission-product yields, and the average absorbable decay energies were therefore emphasized. Other applications, sucl as dose $z+$ the analysis of some decay heat experiments now in progress that separate the beta and gawena heat $\mathrm{xg}$, may require more detalled decay data. in partic". $A r$, as noted in Rei. 1, there is a need to $e^{-}$.a the internal conversion coefficients, and tiiss is expected for Version $v$ in addition to other increased detail on decay data. ENDF/B-IV files now have coefficients for 38 auclides; more information is needed.

As noted in Sec. IV, the total garra energy in Table VII is actually a transition energy; it includes any internal conversion energy. In order to extend the usefulness of Table VII and to provide the user with some guidance on internai conversion energies, we have tabulated in Table VI the fraction of $\bar{E}_{\gamma}$ which is actually internal conversion energy. Most of this table is abstracted from Ref. 9. Fractions for the 38 nuclides having coeffictents in ENDF/B-IV have been calculated and Included; these are identif ted in Table $v I$. The conversion energies generally include the associated $x$ ruys.

The intermal conversion and total gat energies Tobias ${ }^{9}$ tabulates are calculated using his confllation of transition energies and intensities and the conversion coefficients of Ref. 10. Although Tobias' Internal converstion energies are nore complete then values in ENDF/B-IV, the actual energtes in his com- 
pliation are not necessarily consistent with ENDF/BIV energies. Of the 154 nuclides listed in Table VI, 6 are not included in ENDF/B-IV $\left({ }^{102} \mathrm{Rh},{ }^{102} \mathrm{Rh},{ }^{103} \mathrm{Pd}\right.$, $126,{ }_{132} \mathrm{Cs}$, and ${ }^{146} \mathrm{Pm}$ ) and, as is evident from blank col.umns in Table VII, 13 of the ENDF/B-1V values are not Included in Toblas' listing. Eight of the remaining nuclides have transition energies which differ by an order of magnitude, or more, from

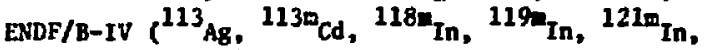
$\left.124 \mathrm{~s}_{\mathrm{Sb}}, \therefore 24 \mathrm{n}_{\mathrm{Sb}}, 166_{\mathrm{Ho}}\right)$, and an additional $18 \mathrm{differ}$ by a factor of two to ten. Nost of the remaining nuclide transition energies are in reasonably good agreenent, and the fractional values of Table VI should therefore be adequate for most users.

\section{v11. CONCLUSION}

Th1s report was prepared $f$ ir use as a convenient reference to a comprehensive set of data for nuclides which are generally classed as fission products $\left(29 \sigma_{z} Z \leqslant 6.9\right)$. l.t contains an abstract (Table VIl) of the nuclide parameters most of ten requested by users, and should be of ut:licy as a gulde co many users desiring wore detall for particular nuclides from WNDF/B-IV files. The gereral content of ENDF/B-IV has been sumarized in Tables $I$ and II. Table VII identif les modes of decay and those nuclides having apectral data, in addicfen to listing nuclide decay and cross-section data. This table is complete for the types of data sumarized (1.e., If no cross sections are listed, there are no crosa-section data for the nuclide in ENDF/B-IV). File errors noted in Sec. $V$ have been corrected in preparing Tabse VII.

Two types of data not given or incoupletc in EKDF/B-IV have been Included. As an atd to the ueer. the $(n, \gamma)$ branching fractions are listed in Table VII, and the conversion election energies are tabulated in Table $U I$ as a fraction of the gaman (transition) energy.

\section{ACKATOWLEGMIITS}

As noted at the beginning of this report, 30 or wore experts fron various Industrial and sovernwent laboratoriea have dtectly contributed in differing degrees to the 2-year tabk force effort. While it is not approprlate to attumt a docuentation of particular contribution or date cources in this abridged data report, it mould be an infustice
If at least the folloring fndividuals vere not acknowledged: C. W. Reich and coworicers at the serojet Nuclear Company; F. Schnittroth at Hanford EngineerIng Development Laboratory; S. Pearletein, O. Ozer, (currently at Electric Power Research Institute), $B$. E. Holdell, and cowozkers at Brookhaven Sational Laboratory. The yield evaluations were essential to developent of the f1sston-product files, bat acknowledgaent of the several Individuals concerned primurily with ylelds properly belongs in another report.

We wish to acknowledge P. B. Hewolg. C. S. Energy Reseazch and Developtent AdeInistration, and $\mathrm{S}$. Fearlstein, Brookhaven National Laboratory, for their support and encouragewent in developing the ENDF/BIV fission-product files.

In adjition, R. B. Mfnogue and S. H. Weiss of the Office of Standards Developrent of the Suclear Regulatory Comelasion proulded part of the support needed in producting this report.

We yould also like to thapk $\mathrm{N}$. 2 . Hhttenore for assistance in preparing tabular data.

\section{REFERENCES}

1. C. K. Rolch, R. G. Helmer, and M. H. Putara, "Radioactive-Nuclide Decay Data for ENDF/B," Aerojet Nuclear Comany report ANCR-1157 (ENDF120) (Auguet 1974).

2. R. E. Schenter and F. Schnttroth, "Radioactive Decay Heat analysis," Conf, on Nuclear Crons Sectione and Tech., Washington, D.C. (March 1975).

3. R. E. Schenter and I. R. Englanü. Huclear Data for Calculations of Radioactivity Effects." Trans. M. Nucl. Soc. 21, 517 (June 1975).

4. H. G. Stmatelates and T. R. England, "FissionProduct Gand Ray and Photoneutron Spectra," Conf, on Muclear Cross Sections and Tech., WashIngeon D. C. (Werch 1975).

5. T. R. England, "An Investigation of Fiseion Procuct Behvior in suclear Restors," Ph.D. Thesis, U. of Uisconsin (1969).

6. L. T. DLliman, 3, Huelear Hedicine, Supplerent Xo. 2, Vol. 10 (Harch 1969), paiphlet No. 4, p. 5.

7. G. T. Garvey, H, J. Gerace, R. L. Jaffe, I. TalI. and I. KeJaon, "Set of Iuclear-Kinss Relations and a Resultant Mass Table," Rev. Yod. Phye. 41, Ho. 4, Part II, S1 (October 1969).

8. A. H. Wapstra and H. B. Gove, Micl. Data Tables A9, Hoz. 4-5, 265 (1971). 
9. A. Toblas, "Data for the Celculetion of Garan Radiation Spectre and Beta Heating from Fission Products (Revision 3)," Central Elecericity Generating Board Research Dapartient, Berkeley thclear Laboratories report RD/B/H2669 CNDC(73)P4 (June 1973).
10. R. 5. Hager and E. C. Seltzer, "Internal CoaverIon Tables. Bart I: $\mathrm{X}$-, L-, M-Shell Conversion Coefficients for $z=30$ to $z=103$," Nucl. Bata A. Vol. 4, Kos. 1 and 2 (Feb. 1968).

\section{TABLE I}

FISSIOA FRODUCT FILES: GESERAL COATEKT

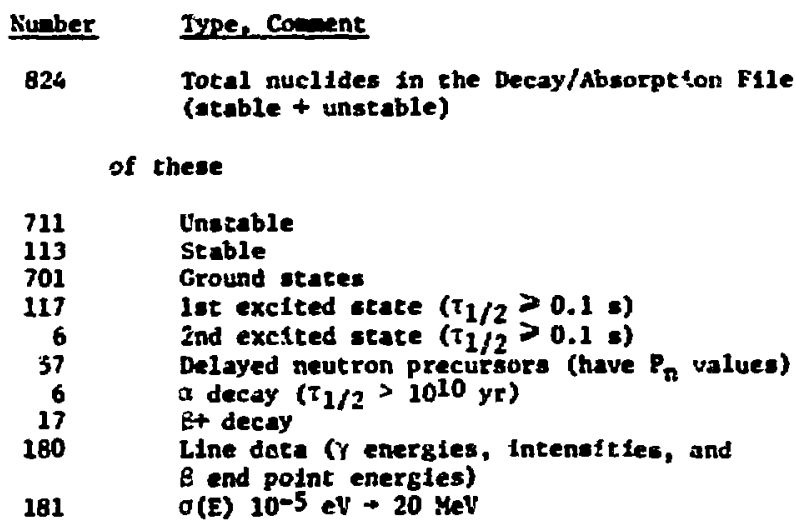

Total $Y$-decay, average 6 and $a$ energies, and branching fractons are glven for all unstable nuckides.

\section{TABLE II}

ENDF/B-IV FISSION YIELE COATEIT (MASSES 72 - 167, CharGes 26 - 70)

Independent yields are given for each of the following ten cases:

No. of Fisstonable

Yiels: Nuelide

Therenl Fast $16 \mathrm{rev}$

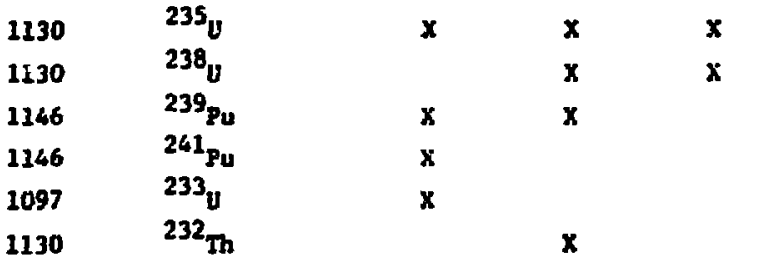

NotE: Ytelds are in the Ganeral Purpose File with the crosssection date for each fissionable nuclide. Decay and crous-section files for the fission products appent cosether on $1 \times$ enctic tapes. The Ceneral Purpose File also contains decey data for 18 nonfisston produces, 16 being actintdea. 


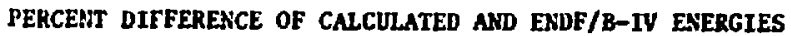

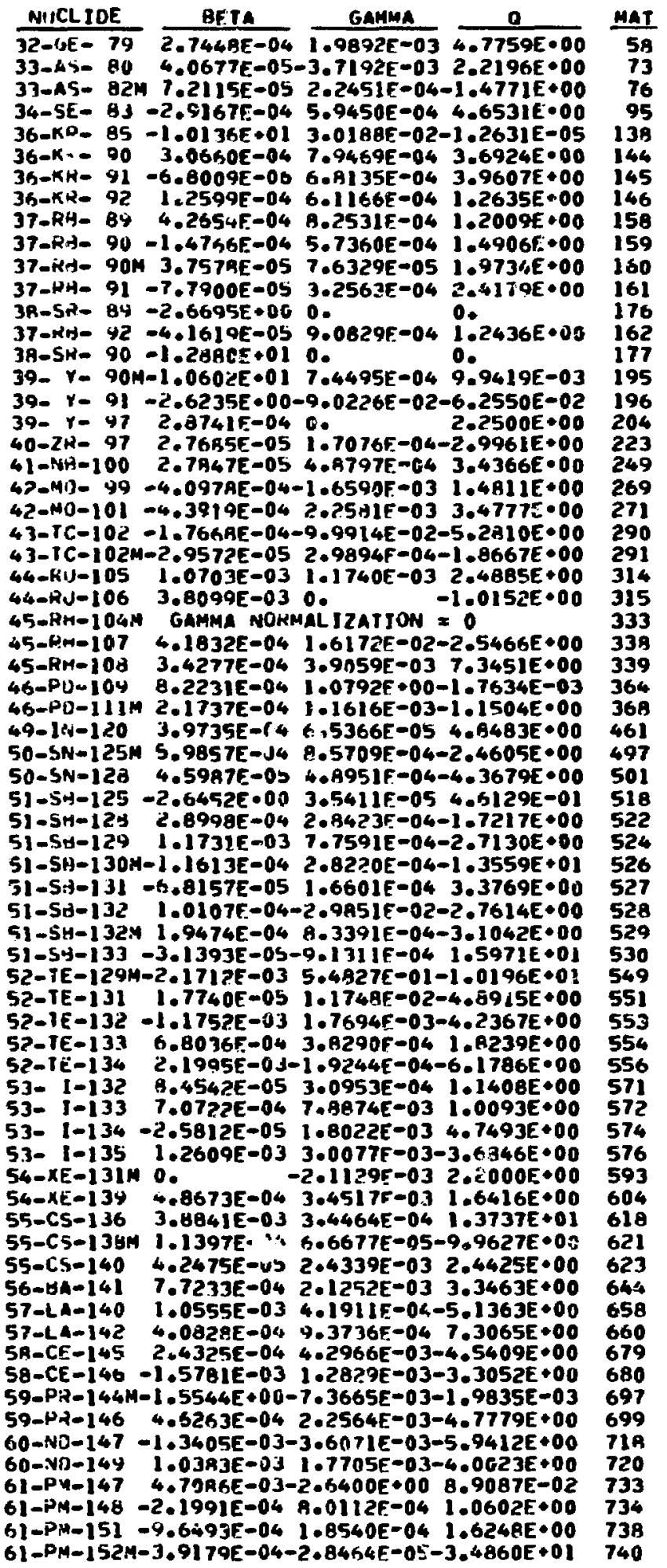


TABLE IV

COAPARISON OP CALCULATFD Q-VALUES vs EMDF/B-IV TABULATIONS

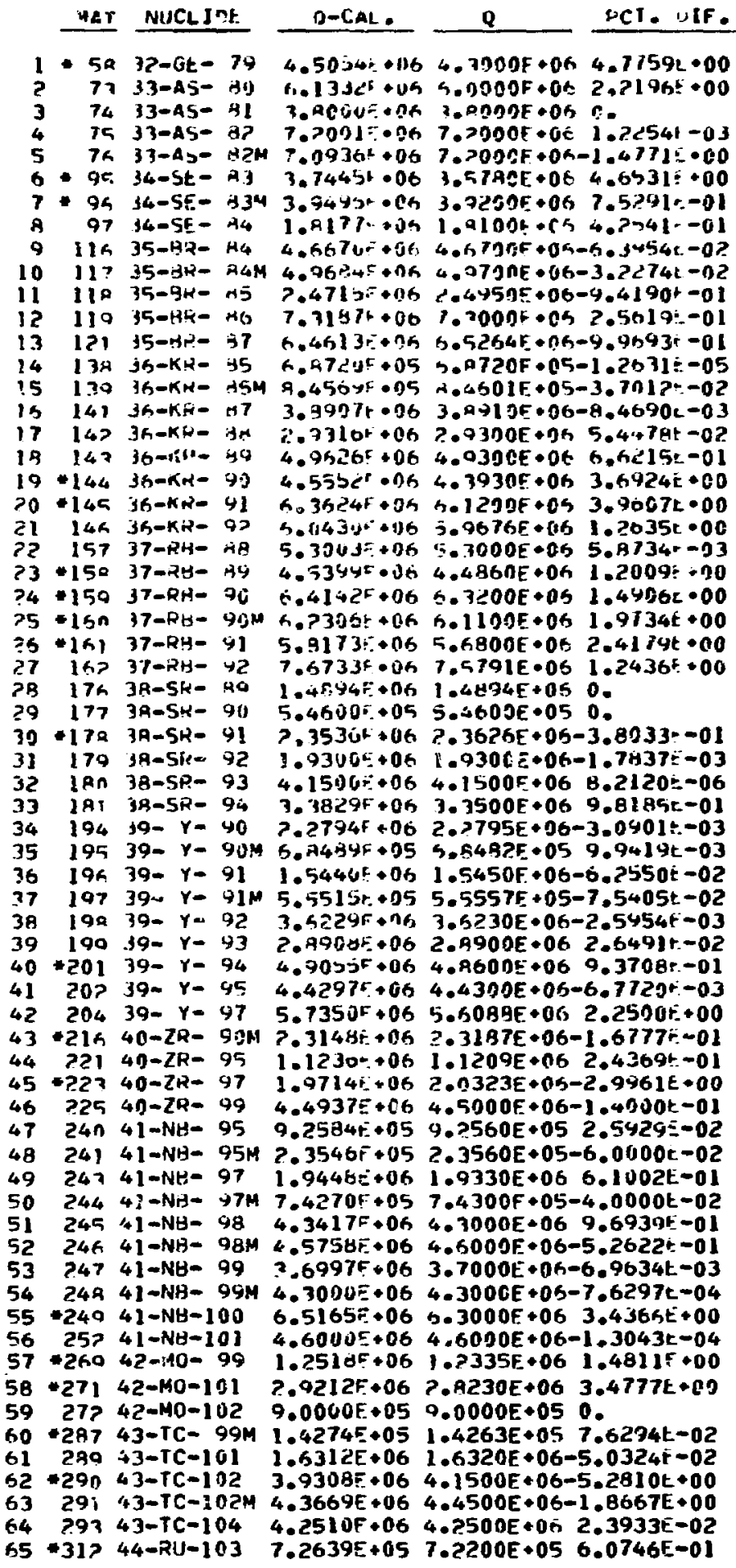


$-3 ! 444-R U-105$

$31544-211-106$

314 44-RU-107

$31746-95 J-19 \mathrm{~A}$

331 45-AM-1 v34

$37245-24-1134$

$33745-M M-104 M$

$37445-7 M-115$

$37545-1 N-105 M$

334 45-Mr-106

$33745-0 \times-105 \%$

370 45-PM-ic7

$33945-17 n-106$

$36 n$ 45-NH-1 AGM

$34745-R+1-10$

344 45 PH-1 I OK

- 3h4 uherij-199

36,5 \&6-p!)-1;1,9:4

3fT $46-\mu(j-111$

36R $4 h-P \mid 1-1114$

39a 67-40-1:744

391 47-AG-111

$397+7-46=1114$

$392+7-5-112$

$45 h+9-\mid v-11=$

45? 47-2V-11FM

44k1 49-14-125

447 49-1:-12un

4.74 jo-Siv-1?5

- 407 bN-5H-1254

400 4.

5ns $3.3-54-1274$

5n) $2 \mathrm{C}-5+2-1 \mathrm{AH}$

snc $59-54-132$

-510 $21-5 \times 4-1 c^{35}$

-5?1 $51-5+1-127$

$5>>1-5-17 A$

5>7 ᄀ1-5̂n-1ㄱM

-5>a >1-5u-1?9

-5\%5 51-54-136

5Pa 51-54-1.30\%

-577 51-5d-131

5Pa jJ-5H-122

$520>1-5 n-1 \times 23$

-53n 5i-5s-133

$53 i>1-5 t-1.74$

$539 ; 1-54-134 M$

-547 52-1 $i-1754$

445 $\$ P-T E-127$

-54A $72-T E-129$

-544 ग2-TE-129M

-551 sz-TE-131

$55 p \div 2-T_{r}-13: 4$

4553 2P-TE-1 $3 \%$

$55452-1 E-133$

555 'รᄀ-Tё-1 $13 M$

5รA $5 Z-T E-134$

-57n 53- 1-131

$45715.7-1-132$

$57>53-1-133$

654 137- 1-136

$57433-1-134 \mathrm{H}$

-574 53-1-135

$57 \% ; 3-1-136$

$57953-1-136 \mathrm{M}$

$595 ; 4-x E-133$

596 T4-XE-1 $33 M$

Son 5G-XE-135
$1.9302 F \cdot 06 \quad 1 . R d 33 E \cdot 06 \quad 2.4885 E+00$

$? .9090 F \cdot 04 \quad 3.9400 E+04-1.9152 t+00$

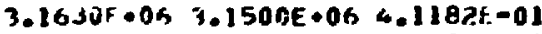

$1.31 .365 .06 \quad 1.3200 E+06-1.0506 t-01$

$3.9750 F .047 .07805 \cdot 040$.

$2.437 u^{2} \cdot 166 \quad 2.4430 F \cdot 06-2.4505 t-01$

GAM:46. HARRAALIZATION =0

5.6456c-05 5.6550F+05-1.5266t 001

1.2970-0.05 1.29708+05 0.

$7.540 u E+06 \quad 3.5400 F+06-7.1544 E-04$

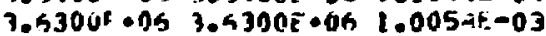

$1.4715 E+05 \quad 1.5100 E \cdot 06-2.5466 t+00$

$4.03305=+06 \quad 4.5000 F=0$ S $7.3451 E \cdot 00$

$4.4300 t \cdot 06 \quad 4.4300 E+06-1.0279 i-04$

$5.799 \cdot 15+06$ 5.4000E+06-2.363!2-03

$5.590 n=06 \quad 5.5000 E \cdot 06 \quad 1.0364 E-02$

$3.027 J F+061.0273 F+06-1.7634 r-03$

1. AR:jJt * $n 5$ 1.98DNE.05 0.

?.1413P.95 ?.1404F.06 5.05405-02

A. $5045=05$-.5090E.05-1.1504t.00

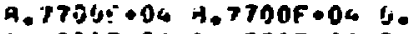

$1.0311=.06 \quad 1.0290 E+06 \quad 2.9785 i-01$

G.500ut - .04 h.5000F-04 D.

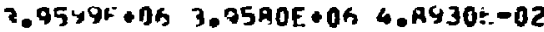

$4.20015 \cdot 16$ \&.PODOF +05 $1.6151 \mathrm{C}-03$

$4.233 n^{2}+06$ 4.7000F +05 B.9J40:-21

$5.557 u t \cdot 0 s$ 5.3600F e of $4.55+83 c \cdot 00$

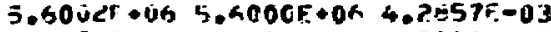

?.362si・04: $2.363 C F \bullet 05-2.3119 r-02$

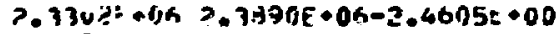

$3.0976=-36$ 3.5,+00E +06 2.4541r-01

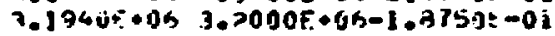

$1.2432 L \cdot 06$ 1.300OF $+G 5-2.3679 E \cdot 00$

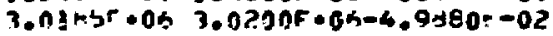

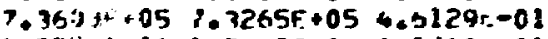

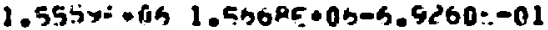

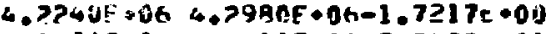

$4 . ? 930=-06$ 4. PhIDF. D T. 7.50R9:-01

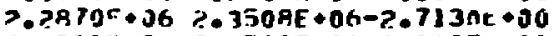

7.0955t-0S $5.0500 E=06$ 9.Pd27t-01

$5.10065 \cdot 06 \quad 3.90005 \cdot 06-1.3559 i+01$

3.502 UP $+0 A$ 3. 3A7GF 0 Gn 3.3769t +00

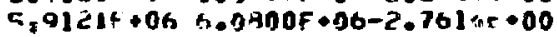

$5.29136 \cdot 06$ h.0400E •0h-3.1 U42E+00

$4.57<2+\cup U S \quad 3.9425 E+061.5971 \mathrm{c} \cdot 01$

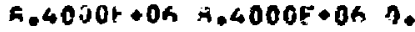

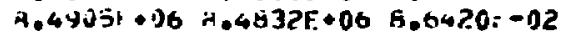

$1.43754 \cdot 05 \quad 1.4473 E+05-6+7+79 t=01$

\%.0534\%-05 $0.0360 E+053.4460 \mathrm{r}-01$

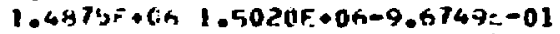

5.HASY $=05$ क.5541E.05-1.01 Y6i..01

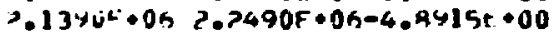

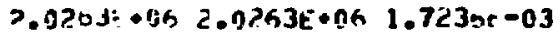

$4.9300=\cdot 055.0500 E+05-4.2367 t+00$

3.014ZEr-OS >.96ODE+0S 1.8239E+00

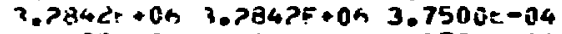

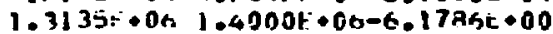

$9.5904+\cdot 05$ 9.4944E •05-8.2142E-U2

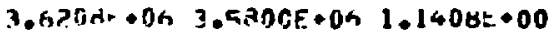

$1.744 \mathrm{fr}+06 \quad 1.7274 \mathrm{~F}+06 \mathrm{~K} 1.0643 \mathrm{r}+00$

4. 34 71F+06 4.1 DONE +06 4.7+93r+00

$3.157 u=\cdot 05$ 3.1570F.05 0.

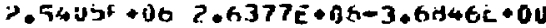

$6.70060+365.3000=05-8 \div 4235 t-05$

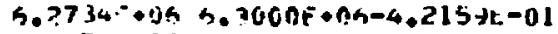

$1.6754 E+05 \quad 1.6393 F+05$ ?. $=000$ r +00

4.2691:-05 4.273nE+155-9.1002t -02

?.3P5YL-05 2.3P90F+05-9.0006=-02

i. 1 1,79F-96 $1.1580 E+04-1.0564 t-02$ 
MAT NUCLIOE

Q-CAL.

$\mathbf{Q}$

PCI. DIF.

$135-60 n j 4-X_{t}-135 \mathrm{M}$

$136 \quad 60>5,4-X E-137$

5.26B2E+05 5.2662E+65 3.8400L-02

$13763354-x E-138$

$4.341 .9 F+064.3470 E+0 G-1.1684 t-01$

$139=604$ 74-XE-134

$139-614 \quad 55-C 5-134$ $2.3463 F+062 . A 300 F+065.7757:-01$ 4. $9601 \mathrm{~F}+06$ 4.A300F+06 $1.64+16 \mathrm{~F}+00$

149 G15 55-CG-? 34M

141 o510 $45-C S-136$

$142 \quad 51925-C 5-137$
$14=620>5-C 5-138$

P.07SHF +06 2.05B5E+06 9.8424c-01

$1.37601 \cdot 051.3760 E+05-1.1624=-03$

$144-621$ iS-CS-13jM

145 6?? $55-C 5-134$

$5521+062.7439 E+061+3137 E+01$

$5.476+1 F+055.4733 E+056.7363 t-02$

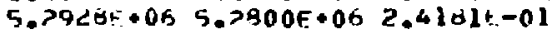

$146 * 623 ; 5-C 5-140$

4. A26UF + 06 5.3600E +06-9.9627E+00

$147 \cdot 54 \pi 56-B A-1374$

148 64> $2 A-13 A-139$

$1496425 B-B A-140$

$4.2975 \mathrm{~F}+064.2900 E+06 \quad 1.7525 E-01$

$6.45345+06 \quad 6.3000 E+06 \quad 2.4425 E \cdot 00$

150 o54 55-HA-141

$151 \quad 545 j 6-34-142$

$152 * 65 Q \quad 57-L A-140$

$153 \quad 650 ; 7-L A-141$

1540650 \7-LA-14ट

1 i5 575 $\supset \mathrm{A}-\mathrm{CE}-141$

$156 \quad 377$ SA-CE-142

$15767 \mathrm{~A}$ SR-CE-141,

$158 \div 6795 B-C E-145$

159 GAR 5A-CE-140

150 695 $59-P_{H}-143$

141696 59-PR-144

162697 j9-PR-144M

$16369359-P_{R}-145$

$16.4 \$ 999$ 59-Pir-14t

$16570 n$ 59-0R-147

166 7ח1 ל9-OR-14B

167 7n? 59-0र-144

$16.8 * 719$ on-NO-147

$169 * 72050-N O=149$

179 -7?? $50-(\mathrm{Ni})-151$

171737 OI-P14-147

$172 * 73451-P M-14 H$

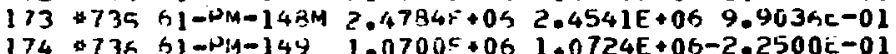

G.

?. $2554 f+06 \quad 2.2540 E+06 \quad 6.3383 t-02$

$1.0326 F \cdot 06$ I. $350 E+06-2.3421 \%-01$

$3.1314 F+06 \quad 3.0300 E+06 \quad 3.3 \div 63 c+00$

$2.1743+06 \quad 2 \cdot 2000 E+06-9.1761 \mathrm{t}-01$

$3.5771 t+06 \quad 3.7708 E+06-5.1363 t+00$

P.42945+05 2.4300E+06-3.1123c-03

$4.347 \mathrm{JE}+06 \quad 4.5170 F+06 \quad 7.3065 \mathrm{0}+00$

$5.3100 \mathrm{~F}+05$ 5.P090F+05 1.AJ63r-02

$1.447 U F+06 \quad 1.4440 E+06 \quad 2.0869 t-01$

$3.1580=+0.53 .1479 E+05 \quad 3.1990 t-01$

$? .3769 E+06 \quad 2.4900 E+06-4.5409 E+00$

$1.0443 F+061.0800 E+06-3.3052 E+00$

$9.3100=+05$ 9.3120E+05-2.1478E-02

$2.9960 r+962.9966 E+05-1.9433 t-02$

$5.04 y 7 F+04 \quad 6+0498 E+04-1.9835 E-03$

$1.9051 \mathrm{t}+0 \mathrm{~K}$ 1.P050E+06 3.3111t -03

$3.9951 F+0 A \quad 4.0800 E+06-4.7779 E+00$

$2.7001 \div .06 \quad 2.7000 E+06 \quad 3.3125 t-03$

4.96 UUF $+106 \quad 4.9690 F+060$.

$2.9993 F+36 \quad 3.0000 E+C 6-2.4500 t-02$

$.4130 F+05 \% .9450 \mathrm{E}+05-5.9412 \mathrm{~T}+00$

$1.61285+06 \quad 1.6800 E+06-4.0023 t \cdot 00$

$2.4844 F+062.4690 E+06 \quad 6.2325 c-01$

$2.2470 F \cdot 05 \quad 2.2450 F+058.9647 t-02$

$3.4911 \mathrm{E} \bullet 052.4650 \mathrm{0}+061.0602 \mathrm{t}+00$

$175 * 732$ 61-PM-151 $1.2073 F+061.1896 E+061.6248 E+00$

$176 \quad 739$ hl-PM-l $52 \quad 3.6070 F+06 \quad 3.6000 E+06 \quad 1.9448 E-01$

$177.74 n$ 5I-PM- IS2M ?.3450F+06 3.6000E+06-3.4860E+01

$178 \quad 742$ ol-PH-153 1.9004F+06 1.8000E+06 2.4570t-02

$179=75062-5 M-153$ 9.0251F+05 $9.0360 E+05-7.5351 L-01$

$190 \cdot 77953-E U-156 \quad 2.434 d F+06 \quad 2.4530 E+06-7.4349 c-01$

*CALCULATEd D DIFFERENCE EXCEEDS UNCERTAINITY 
TABLE v

NUCLIMES HAUING TYPOGRAPHICAI OR SUSPECTED ERRURS IN ENDF/B-IV FISSIOH-PRODUCT FILES

\begin{tabular}{|c|c|c|}
\hline Nuclide & $\begin{array}{l}\text { Corrected in } \\
\text { Table VII } \\
\end{array}$ & Comments \\
\hline $97 \mathrm{Y}$ & yes & $\bar{E}_{y}=9.35 \times 10^{5} \mathrm{ev}$ \\
\hline $104 m_{R h}$ & yes & $\begin{array}{l}\text { Hermalization factor }(F)=0 \text { (F should be } \\
\left.1.8535 \times 10^{-2}\right) \text {, and typographical errors in } \\
\text { spectra. }\end{array}$ \\
\hline 12 & yes & $\tau_{1 / 2}=3.15569 \times 10^{12} \mathrm{~s}$ \\
\hline & yes & $\begin{array}{l}\text { Internal conversion energy of } 0.6682 \times 10^{5} \mathrm{eV} \\
\text { added to } \overline{\mathrm{E}}_{\gamma} \text {. }\end{array}$ \\
\hline${ }^{130 m} \mathrm{sb}$ & yes & $\begin{array}{l}\vec{E}_{\gamma} \text { too sma11 ( } E_{\gamma} \cong 3.04 \times 10^{6} \mathrm{ev} \text { ). Change } \\
\text { normalization to } 1.17717 \text {. }\end{array}$ \\
\hline${ }^{131} \mathrm{Sb}$ & yes & $\bar{E}_{\gamma}=1.7025 \times 10^{6} \mathrm{ev}$ \\
\hline${ }^{133} \mathrm{Sb}$ & yes & $\begin{array}{l}\bar{E}_{Y} \text { too large }\left(E_{Y} \cong 2.5 \times 10^{6} \mathrm{eV}\right) \text {. Change norm- } \\
\text { alization to } 3.87351 \times 10^{-1} .\end{array}$ \\
\hline${ }^{136} \mathrm{Cs}$ & no & $\overline{\mathrm{E}}_{\gamma}$ includes some $\gamma$ energy from ${ }^{136} \mathrm{Ba}$. \\
\hline & yes & $\begin{array}{l}\bar{E}_{\text {q }} \text { too small }\left(\overline{\mathrm{E}}_{\gamma} \cong 2.6 \times 10^{6} \mathrm{eV}\right) \text {. Change } \\
\text { normalization to } 1.23827 .\end{array}$ \\
\hline $140_{\mathrm{La}}$ & yes & $\begin{array}{l}\overline{\mathrm{E}}_{\gamma} \text { too small }\left(\overline{\mathrm{E}}_{\zeta} \cong 2.3 \mathrm{v} 10^{6} \mathrm{eV}\right) . \text { Change } \\
\text { normalization to } 1.03275 .\end{array}$ \\
\hline${ }^{142} \mathrm{La}$ & yes & $\begin{array}{l}\bar{E}_{\gamma} \text { too large }\left(E_{\gamma} \cong 2.4 \times 10^{6} \mathrm{ev}\right) \text {. Change } \\
\text { normalization to } 0.96470 .\end{array}$ \\
\hline${ }^{152} \mathrm{~m}_{\mathrm{Pm}}$ & yes & $\begin{array}{l}\bar{E}_{\beta} \text { too small }\left(\bar{E}_{\beta} \cong 0.9 \times 10^{6} \mathrm{ev}\right) \text { and beta in- } \\
\text { tensities do not sum to } 1.0 . \text { Chenge normal- } \\
\text { ization to } 2.14551 .\end{array}$ \\
\hline${ }^{166} \mathrm{Er}$ & yes & $\sigma(0.0253 \mathrm{ev})$ too large $(\sigma \cong 20 \mathrm{~b})$ \\
\hline
\end{tabular}

TABLE VI

INTERNAL CONVERSION ENERGIES (EXXRESSED AS FRACTIONS OF TRANSITION ENERGY)

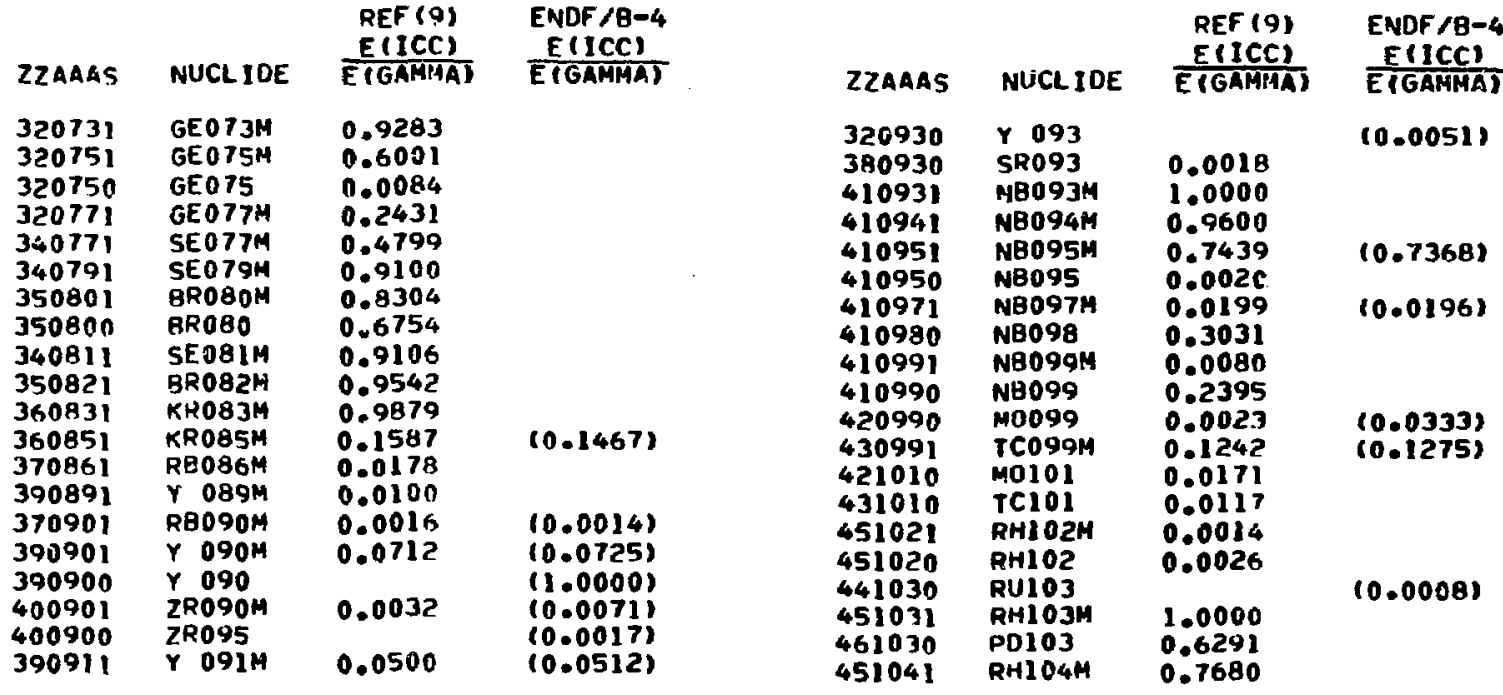




\begin{tabular}{|c|c|c|c|c|c|c|c|}
\hline ZZAAAS & NUCL IDE & $\begin{array}{l}\text { RE (9) } \\
E(1 C C) \\
E(G A M A A)\end{array}$ & $\begin{array}{l}\text { ENOF/B-4 } \\
\frac{E(I C C)}{E(G A M P A)}\end{array}$ & ZZARAS & NUCL IDE & $\begin{array}{l}\text { REF (9) } \\
\text { E(ICC) } \\
\text { E(GAMAA) }\end{array}$ & $\begin{array}{l}\text { ENDF } / B-4 \\
E(I C C) \\
E(G A M M A)\end{array}$ \\
\hline 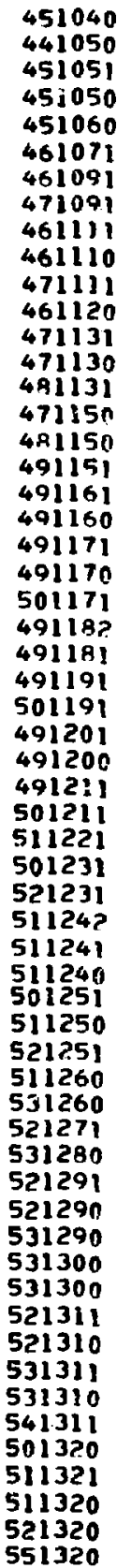 & 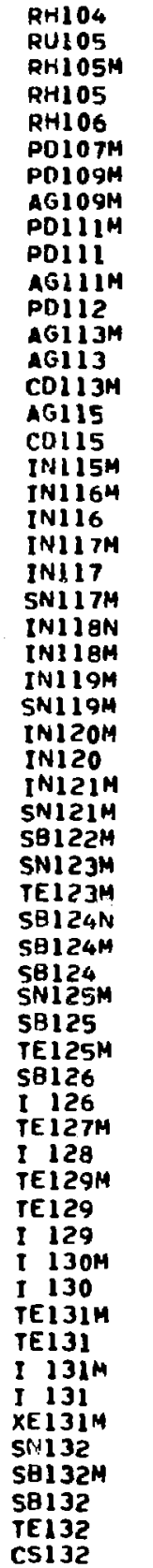 & 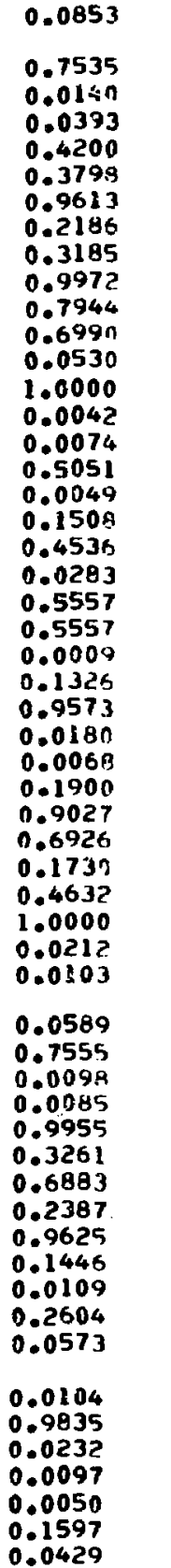 & $\begin{array}{l}(0.9804) \\
(0.0358) \\
(0.2365)\end{array}$ & 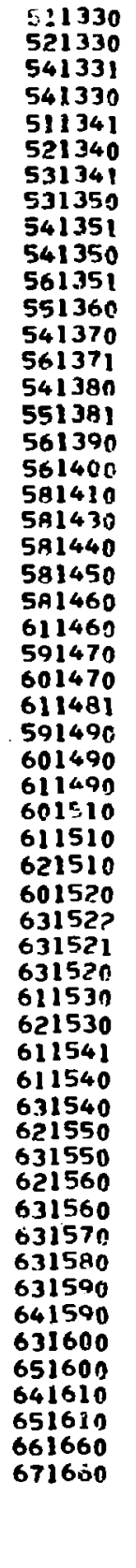 & 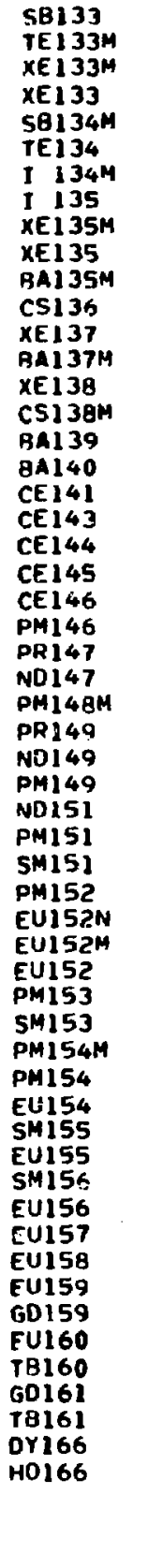 & 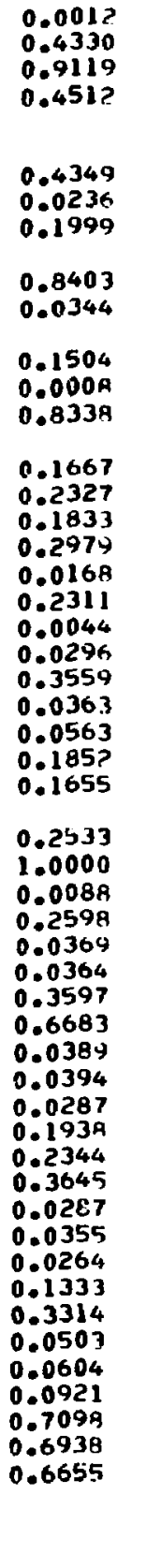 & $\begin{array}{l}(0.8969) \\
(0.6275) \\
(0.0326) \\
(0.0520) \\
(0.1883) \\
(0.0614) \\
(0.0092) \\
(0.1007) \\
(0.1788) \\
(0.1741) \\
(0.1839) \\
(0.4457)\end{array}$ \\
\hline
\end{tabular}




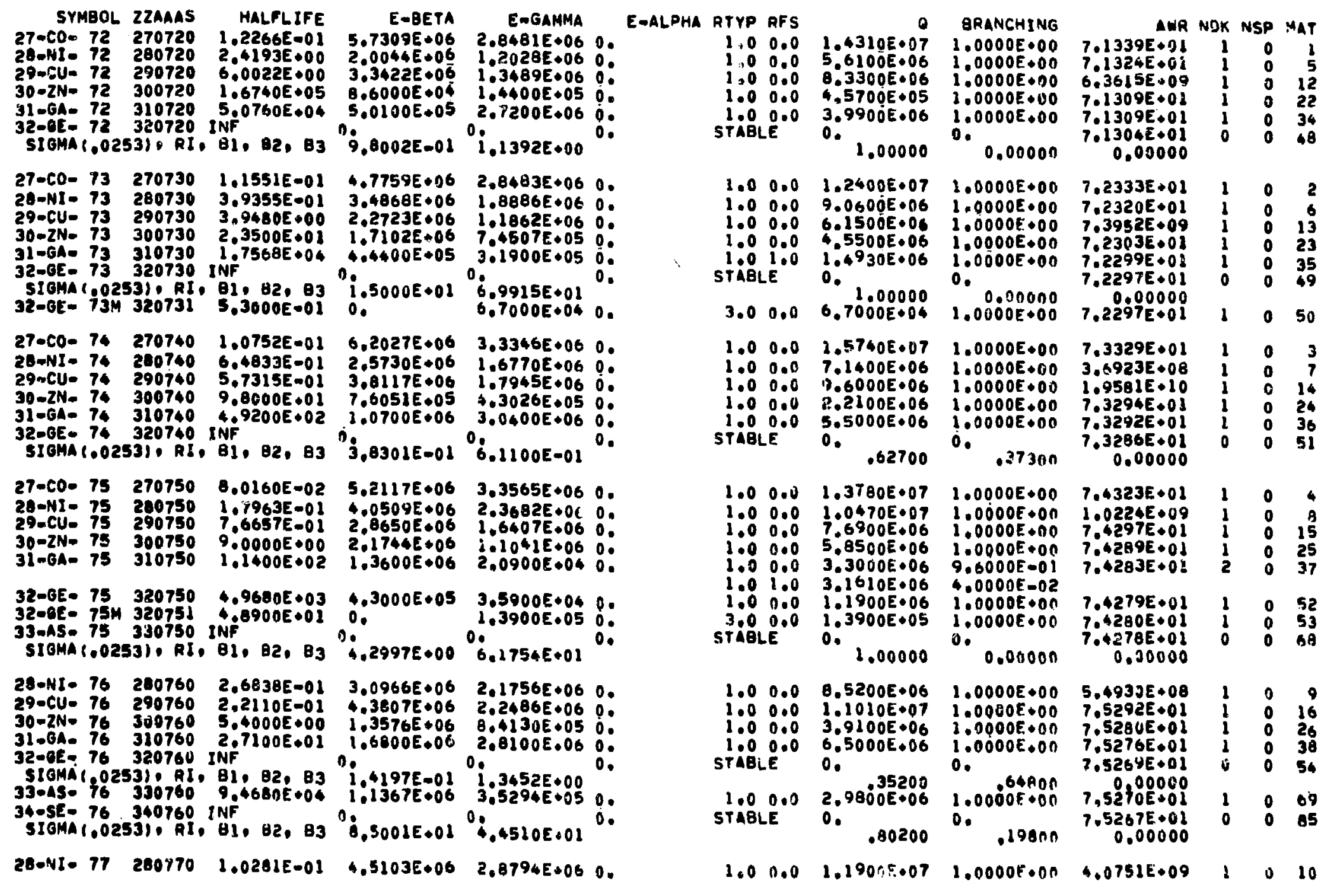




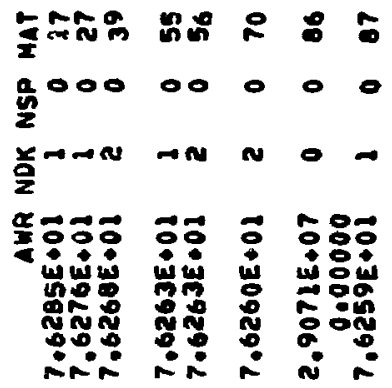

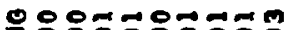

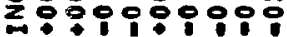

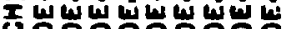

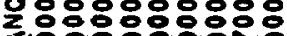

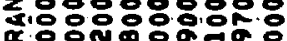

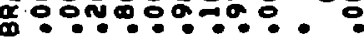

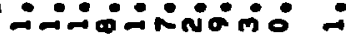

- 옹옹

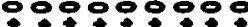

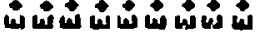

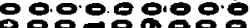

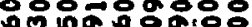
ONn

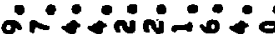

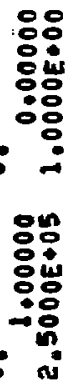

no00000000

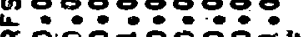

00000000000 2

$$
\begin{aligned}
& 8 \\
& 8 \\
& \text { i }
\end{aligned}
$$

:

8

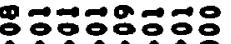

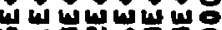
$\varphi \rightarrow 8 x+4=$ 20

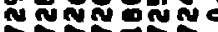

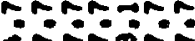

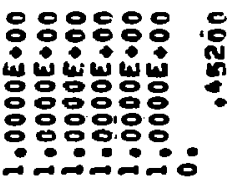

두웅요

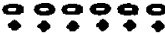

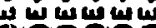

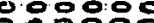
8 \& กับ

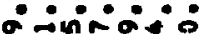

$\frac{\Phi}{1}$

$\frac{1}{3}$

$\omega$

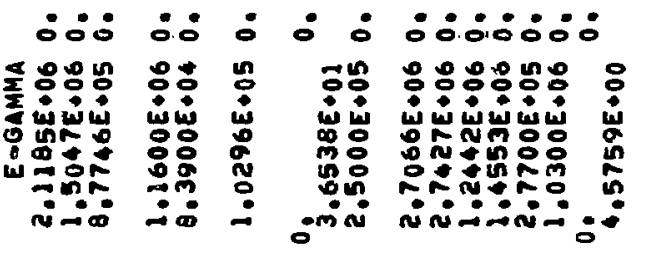

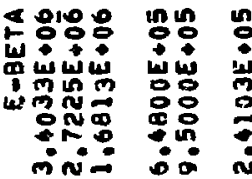

w0웅 $\rightarrow$ w. 눙요 충웅음

ivi

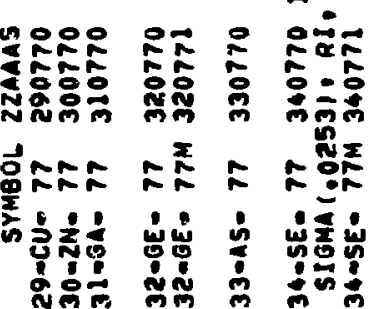
우웅요 4 누요으응

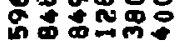
$\checkmark$ $-6$

90:

ㄴ

용

$-\frac{1}{2}$

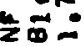
misina:

소요 1. 응ㅇㅇㅇ 동용요운 $\because 00$ inis 0000000\% DQPQER S응요으. 政

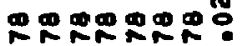
1.1 .5

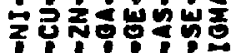
$\operatorname{8} \dot{0} \dot{0} \dot{0} \dot{0}$
웅ํㅇㅇ 山嵌山㟧

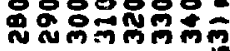

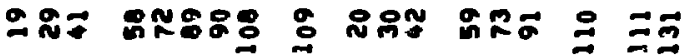

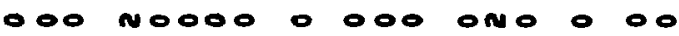

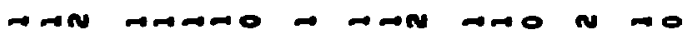

훙ㅇㅇ 형ㅎㅇ영

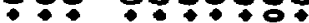

는

ais

$0 \times$

in山wis

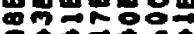

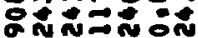

\%ญํำ

in:

iñin

900

웅ㅎㅇㅇㅇㅇ

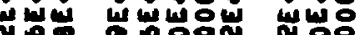

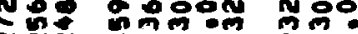

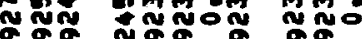

in Ran a in

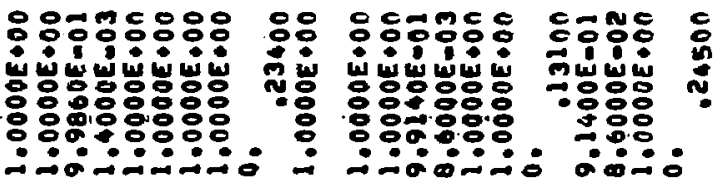

n.0\%90m

웅ㅇㅇ은

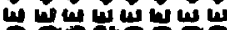

и

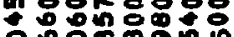

○.

-Divinis

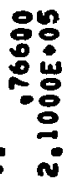

우웅웅

000

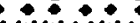

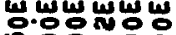

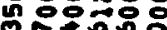

ज

$3000=10$

80웅

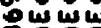

00

$\rightarrow 08$

010

i்

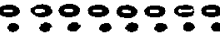

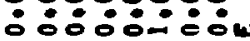

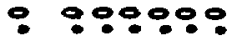

웅

웅ㅇㅇㅇㅇㅛ

:형․

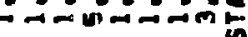

$\div 0$

$\circ 89$

(

แ능

우웅

บ :

n:

009

$89 \%$

$\omega$ w

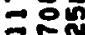

에 N

$m$ in

웅ㅇㅇㅇ

in

$+ \pm 8$

-

$\rightarrow \operatorname{ci}$

웅

용요

옹응

a a

R.R

논

10

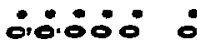
영영 눙영

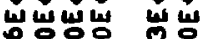

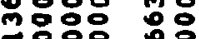
승ㅇㅇ용요 i-:0

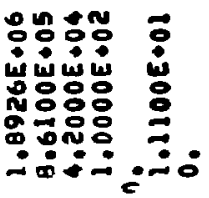
오ㅇㅝㅗㅇ 우웅 แ山山س 용휴 응

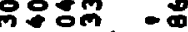
- ivinza.

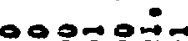
웅우웅 옹ㅇㅇ우웅 ชูm o o I RRRRR

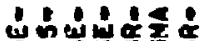

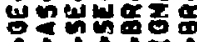

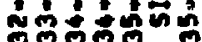

$\div: 0$

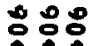
in m in 0 NoN m. 098 u以 숭요 in ini 웅웅ㅇㅇ 네의 om. $\Rightarrow=$

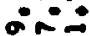

웅요

옹요

808

옹용

눙웅

룽ํำ

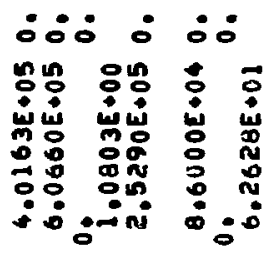

웅ㅎㅇ영

u w

요 9

중요

in :in

며 लि

山端

봉 눙

유 -

N-4 $=$

영우웅

웅요

옹오웅

लm

$$
\text { 怘。 }
$$

000 क

$\therefore$ in 
SYMBOL ZZAAAS

29-CU- 81290810 $30-2 \mathrm{~N}-81300810$

$31-6 A-91310810$

32.GE - 81320810

33-AS- 81330810

34-SE. BI 340810

36-5E- $81 \mathrm{M} \mathrm{340011}$

35-8R- 81350010 INF

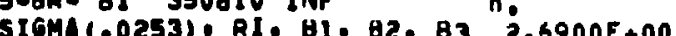

$360 k$ - 36010100.6276501200

36-KR. BIM 360811 $1.3300 E_{0.01} 0$.

38-2N- $12 \quad 300820 \quad 1.3526 E-02 \quad 3.7891 E 006$

$31-64-82 \quad 310020 \quad 1.5376 E=01 \quad 4.7596 E \cdot 06$

32-6E- 82 320020 $4.6000 E+00 \quad 1.2179 E+06$

33-AS. 82 330820 1.9000E•01 3.2109E.06

33-1S- 02M 330821 1,3300E+0I $1.8192 E 006$

SIOMA (0253), RI, B1, 82, 日3 4,5002E=02

35.BA- 82M 350821 3,6600E•C12

$4,5002 E=02$

$2.7424 E \bullet 04$

$36-K A-92360820$ INF

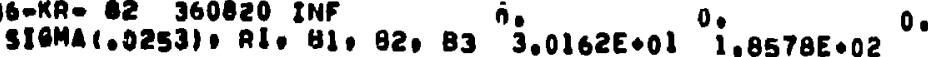

30-2N- $03300030 \quad 0.3858 E-02 \quad 4.6943 E-06 \quad 3.5324 E+06 \quad 0$

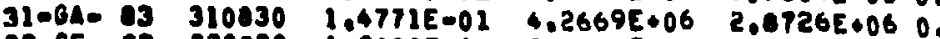
32-6E- O3 320030 $1,9006 E+00$ 3.0366E-06 3.0042E.06 0.

330AS- $03 \quad 330030 \quad 1.3500 E+01 \quad 1.6763 E+06 \quad 9.0446 E \cdot 05 \quad 0$.

34-5E- 03 346330 1,3500E-03 4.6106E.05 2.5592E.06 0. 36-5E- O3M 360031 $9.0000 E \cdot 01$ 1.3017E॰06 9.0033E०05 00

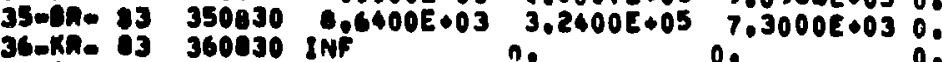

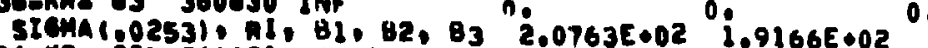

36-Ka- O3m 36003i 6.6960E:93 0.

$31-04=04310040 \quad 9.0073 E=02 \quad 5.1650 E+06 \quad 3,3800 E+06$ O. 32-OE- OA 320040 1,2000E+0Q ?.0212E•06 1.9135E+06 O.:

33-AS- $4330040 \quad 5,0000 E \bullet 00 \quad 3,7612 E+06 \quad 2,1036 E+060$.

34-5E- O4 360840 1.9000E.02 5.3084E.05 4.07YOE+05 O.

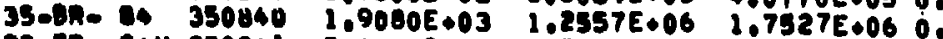$$
\text { RT } 1
$$$$
\begin{array}{ll}
1.0 & 0.00 \\
1.0 & 0.0 \\
1.0 & 0.00 \\
1.0 & 0.00 \\
1.0 & 0.0 \\
1.0 & 0.00 \\
3.0 & 0.00
\end{array}
$$$$
\text { STABLE }
$$$$
\text { STABLE }
$$

2.00 .0

3.00 .0

1.00 .0

1.00 .0

1.00 .0

1.00 .0

1.0000

STABLE

1.00 .0

1.00 .0

3.00 .0

STABLE

1.00

1.00 .0

1.00 .0

5.00 .0

1.00 .0

1.01 .0

1.00 .0

1.01 .0

STABLE

strale

3.00 .0

1.00 .0

1.00 .0

5.00 .0

1.00 .0

5.00 .0

1.00 .0

1.00 .0

1.00 .0
0

$1.4410 E \cdot 07$ $1.1580 E \cdot 07$ $7.4400 E+06$ $5.6500 E+06$ $3.8000 E 006$ 1. 5A00E.06 $1.0300 E \cdot 05$

.09700

$3.0000 E 005$

$1.0630 E+07$

$1.2350 E+07$

$3.580 Q E \cdot 06$

7.2000E-06

0 .

87100

$3.0900 E 006$

$3.1360 E+06$

$4.6000 E_{0} 04$

.55600

$1.2920 E \cdot 07$

$1.2420 E+07$

$0.4900 E 006$

$3.7501 E .05$

$4.8300 E+06$

ధ.5800E.06

$3.5700 E+06$

3.9500E+06

0.

1.00000

$4.18000_{004}$

$1.37105 \cdot 07$

$7.5400 E \cdot 06$

$3.3864 E 006$

$9.9900 E_{006}$

$6.7577 \mathrm{E} .05$

$2.8100 E 006$

0.97000 .005
BRANCHING

$1.0000 E+C 0$ $1.0000 E+00$ $1.0000 E+00$ $1.0000 E+00$ $1.0000 E+00$ $1.0000 E+00$ $1.0000 E+00$ $.9030 n$ $1.0000 E+00$ $1.0000 E+00$

$1.0000 E+00$ $1.0000 E \bullet 00$ $1.0000 E+00$ $1.0000 E \bullet 00$ $1.0000 E+00$

.12900

$1.0000 E+00$ $2.4000 E-02$ $9.7600 E_{-01}$

$$
.44400
$$

$1,0000 E+00$ $1.0000 E+00$ 9.9840 E-OL

$1.6000 E-03$

$3.3000 E=01$

$6.4000 E=01$

$1.0000 E+00$

$1.0000 E+00$ $1,0000 \varepsilon+00$

0.00000 $1.0000 E+00$

$1.0000 E 000$ $9.0400 E=01$ $9.6000 E-02$ 9.9870 Eal $1,3000 E-03$ $1.0000 E+00$ $2.0000 E .00$ 2.0000 teon
AWR NOK NSP MAT

$8.0269 E+01<021$ $8.0253 E+01 \quad 1 \quad 0 \quad 31$ $0.0241 E+01 \quad 1 \quad 0 \quad 43$ $4.0798 E+08 \quad 1 \quad 060$ $8.0227 E .01 \quad 1 \quad 174$ $0.0233 E \circ 01$ B.022JE+0I 0 O Ii2 0.00000 $0.0222 E+01 \quad 1 \quad 0132$ $8.0222 E+01$ I 0133

$0.1249 E+01 \quad 1 \quad 0 \quad 32$ 8.1237E+01 I 0 44 $0.1224 E+01 \quad 1 \quad 001$ B.1221E+01 1276 $0.1213 E+0$ 0,00000 $0.1213 E+01$ $4.5325 E \bullet 09$

$8.1210 E+01$ 0.00000

$3,9417 E+95$ $0.2233 E+02$ $2,0012 E+0 B$

$0.2212 E \bullet 01$

$0.2207 E+01$ $0.2207 E \circ 0$

$0.2203 E+01$

$8.2202 E .01$ 0.00000 $B .2202 E+01$

$8.3230 E+01$ $8.3215 E \bullet 01$

$0.320 I E+01$

$0.3197 E+01$

$4.5087 E 008$

$2.3289 E+08$
009

$\begin{array}{lll}1 & 0 & 113 \\ 2 & 0 & 114\end{array}$

$0 \quad 0234$

1033

20045

2077

295

10215

$0 \quad 0135$

10136

1046

297

12110 


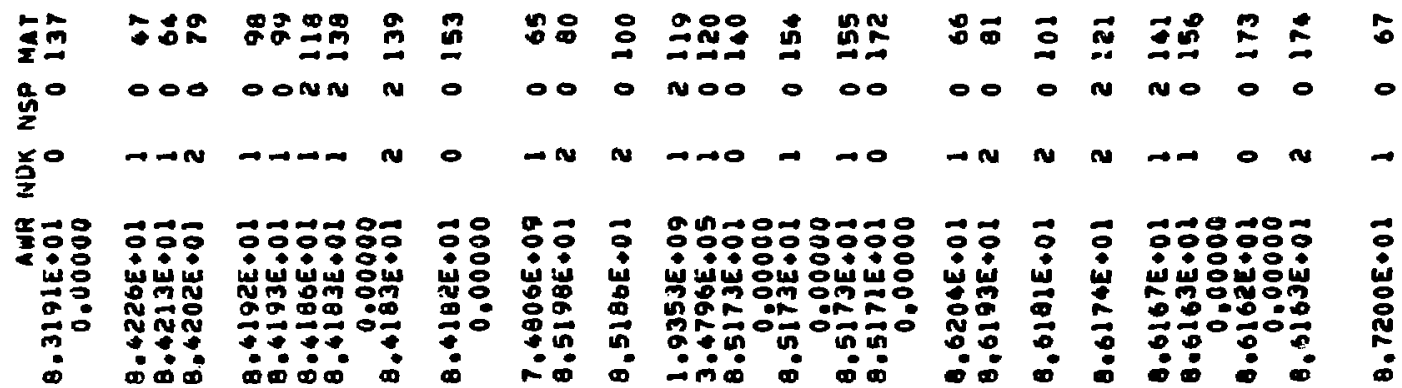

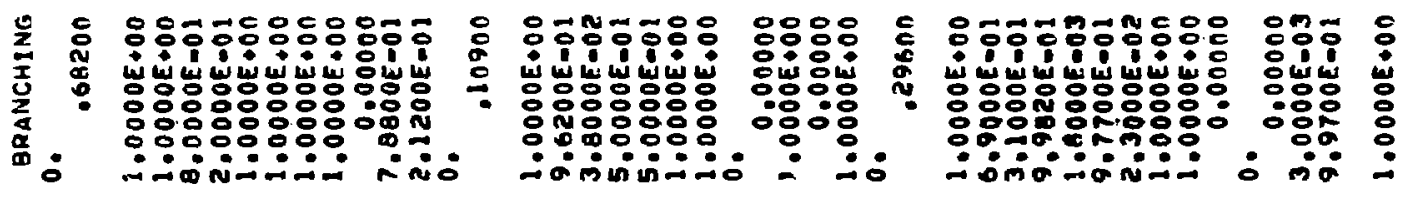

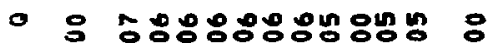

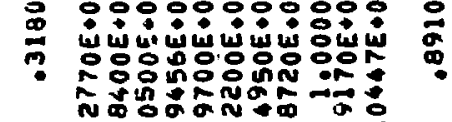

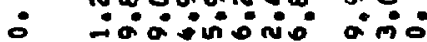

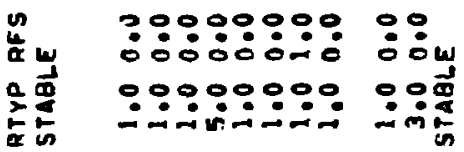

눙유유유유.

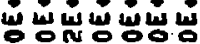

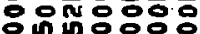

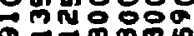
융요융 o in in : $\pi-8$ $00000000 \%$

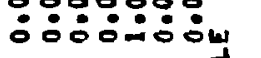
:ㅇ:윰요

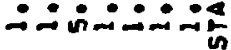

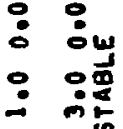

둥융유융웅

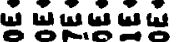
우응요 공요요

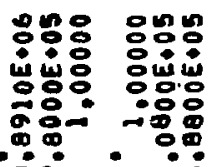

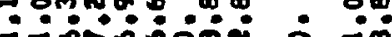

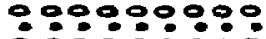
- 00.00000 000000000 -.6.0.9.

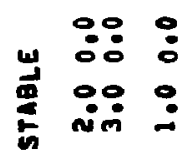

$\frac{1}{2}$

$\frac{1}{3}$

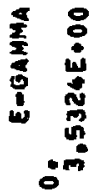

:0:

¿:0:0:

$\div \quad: \div$

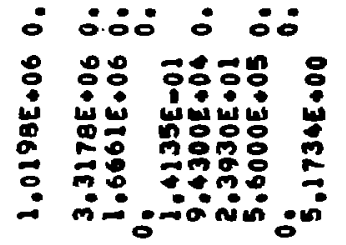

$\div:$

용

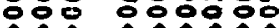
㟧 iving ing

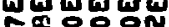

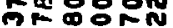

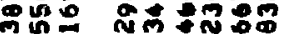

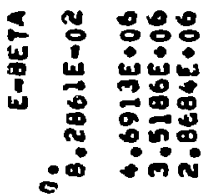
$\because-1000-1$

$\ddot{\omega}$

ก

5 :0 - in in

웅영용

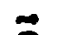

:

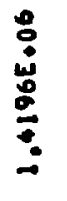

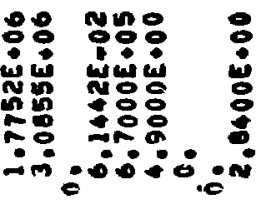

:웅 is

훙

88 in

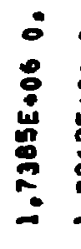
u仙的

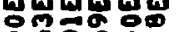
웅요웅 जंजिंजि

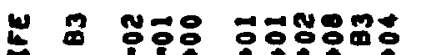

-

ป

$2=$

.

i

$\geq 6$ w岁

동

山山山- -

ㅇㅇㅇ뭉

8요용

$\because \because \div \dot{0}=$

(

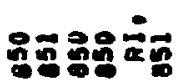

영요

웅요요

ㅇㅇㅇㅇㅇ응

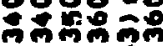

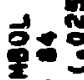

ตัn

题=

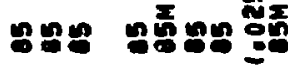

눙은

में

50

$\Rightarrow$ ․․․

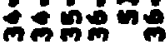

๓

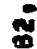

눈

จ용

w

88

궁

웅 8

후웅

옹

옿형

5

88

총 눙옹 슨

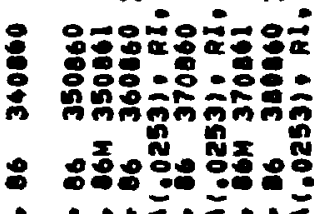

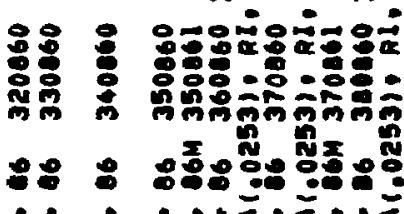
웅

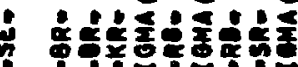

+
:

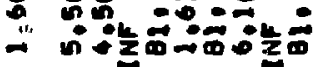

00

㟧

車

$2=$

$\because 8$

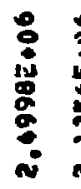

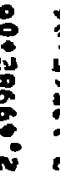

8

8

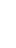

8

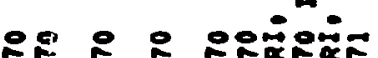

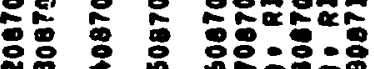

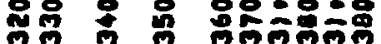

तx

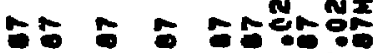

4

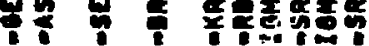

ㅎํㅇ
:

:

$\dot{H}$

8

i

$\circ$

:

兽

in

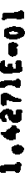

\$

8

:

8 


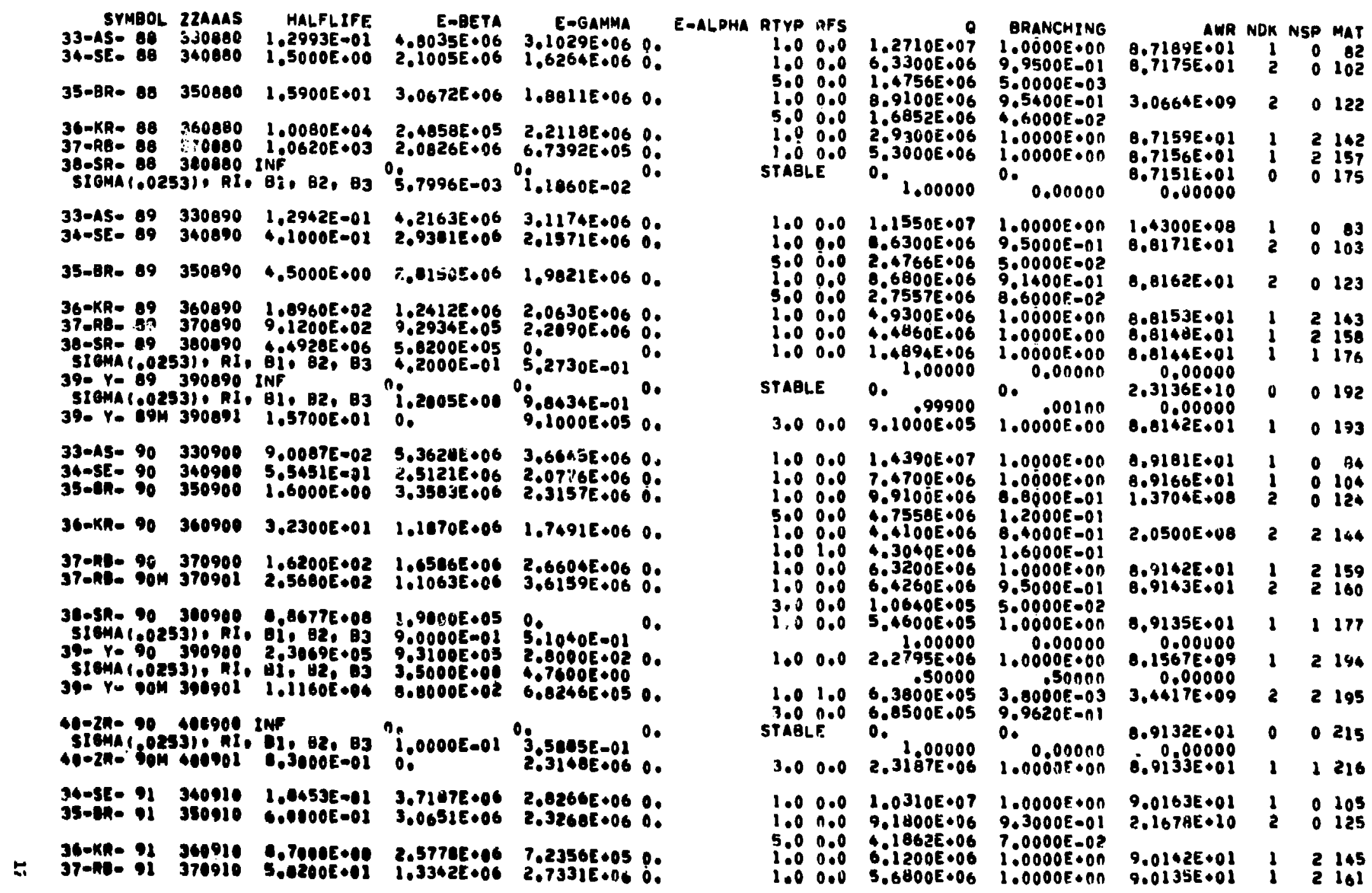




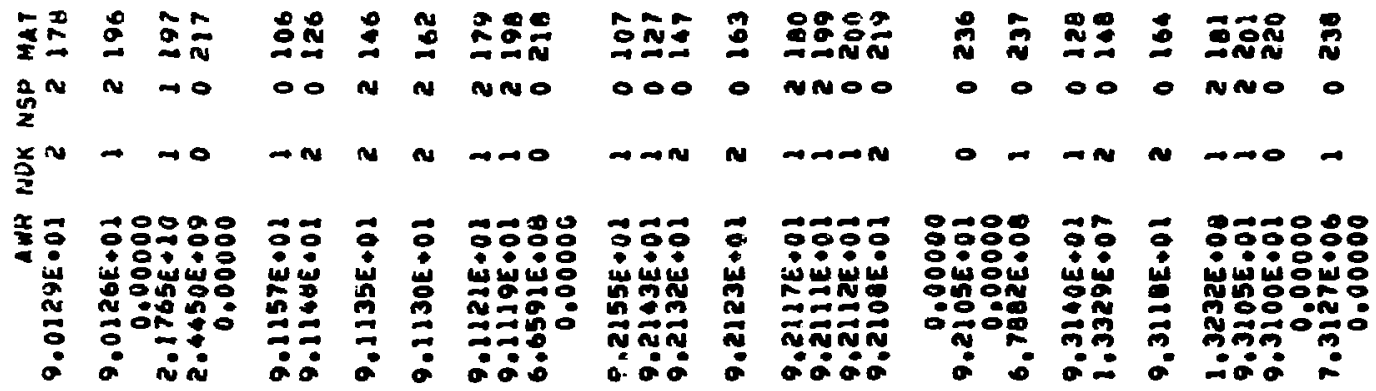

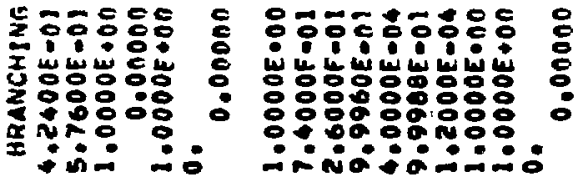

3

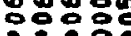

嵌岩

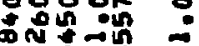

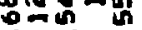

iñ ni:

no:0 0

这: :

0000 of

요요

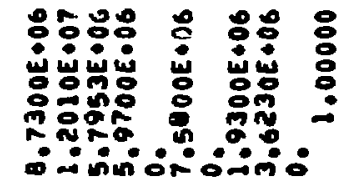

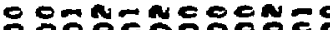

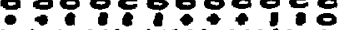

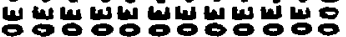

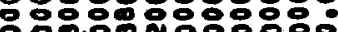

요요용ㅇㅇㅇㅇㅇㅇ

08040.0800

-...…ㄴ.

융요

동ㅇㅇㅇㅇㅇㅇㅇㅇㅇㅇㅇㅛ

ش

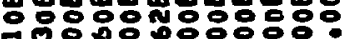

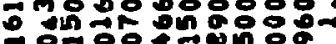

응요

으읃ㄷㄷㅇ

88:89:웅

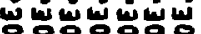

8요요용

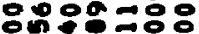

$9: 0 \div 9: 0 \%$

- innominutón

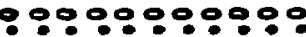

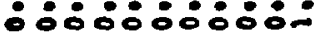

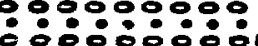

0000000001

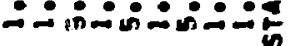

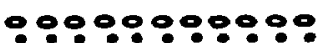

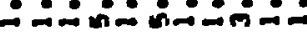

유유.:

둥요융요

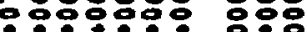

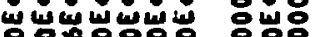

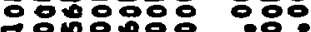
궁응우웅 MNㅔㅇㅛ -0iánis: n

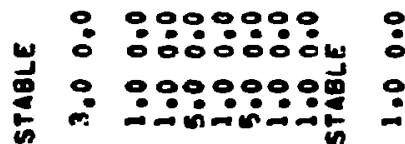

I

$\frac{1}{1}$

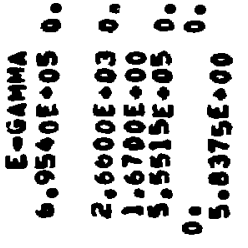

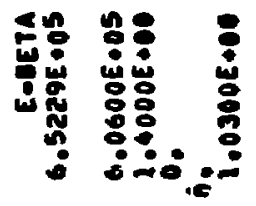

녕요용

这?

눙

옹효

우울

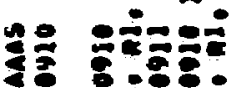

18

8. 83.

수

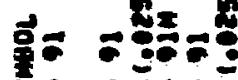

60

$8 \%$

눙

8

응

in

i:

i:

80

눙

$\div 8$

$8:$

옹

83

36

ct

.

8

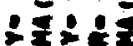

당

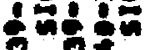

8

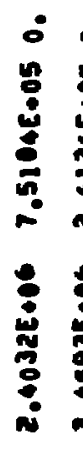

:

:0:

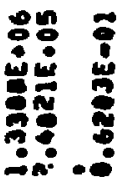

$\rightarrow \infty$

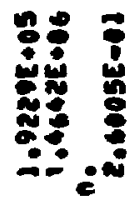

ms

8

눙

- 웅․․

- +5.5

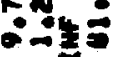

8

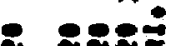

- 38후으.

- $838=$

- 2 cast

- \& ave.

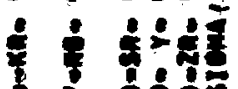

3 \& 880
:0: : 0000

유:융

눈단

추으.

:5:

in

:

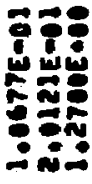

울용

is?

ase

$4+2$

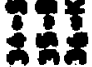

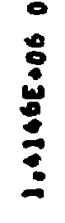

웅ㅇㅇㅇㅛ

ज山w․

우웅요

혀유:

-onin
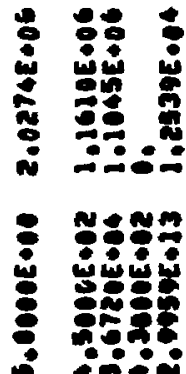

Nenm

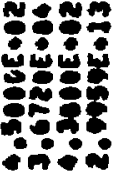

7.8.

mon

호요옹

8883

$\cos$

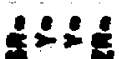

1.8

int?
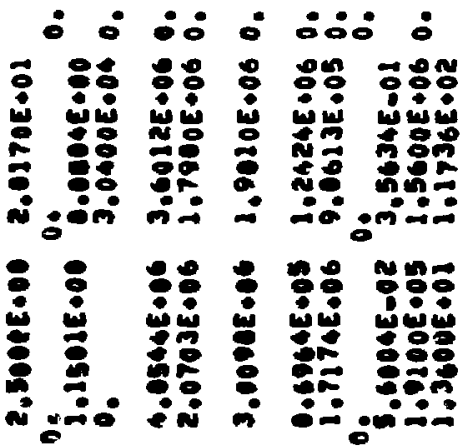

ar

30

i

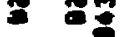

An

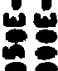

82

$-20$

$\therefore$ 웅룽

$-2=0$

도웅은

* Pr

ger

19:

453
88

8 ह

$28=328 \%$

눙

$3+2500$ 


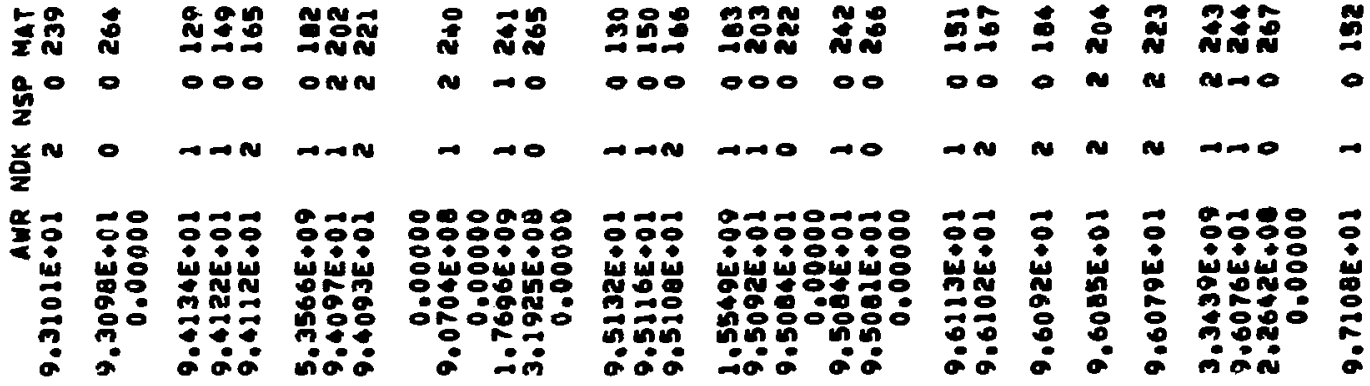

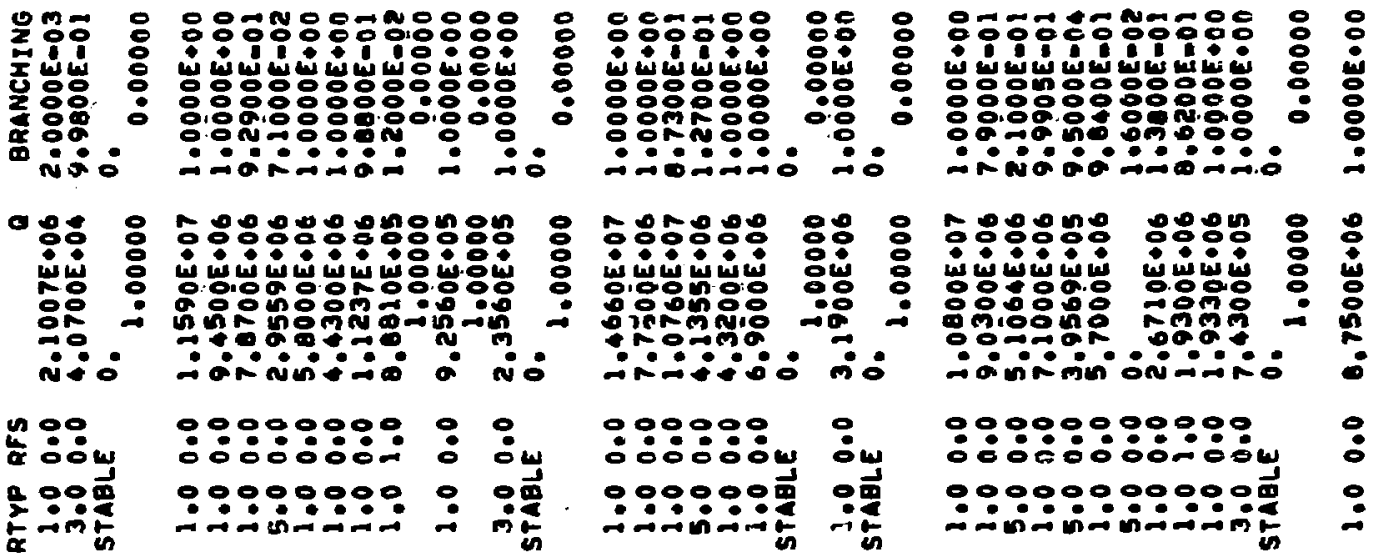

$\frac{5}{3}$

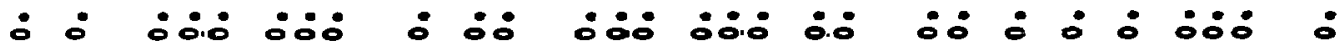

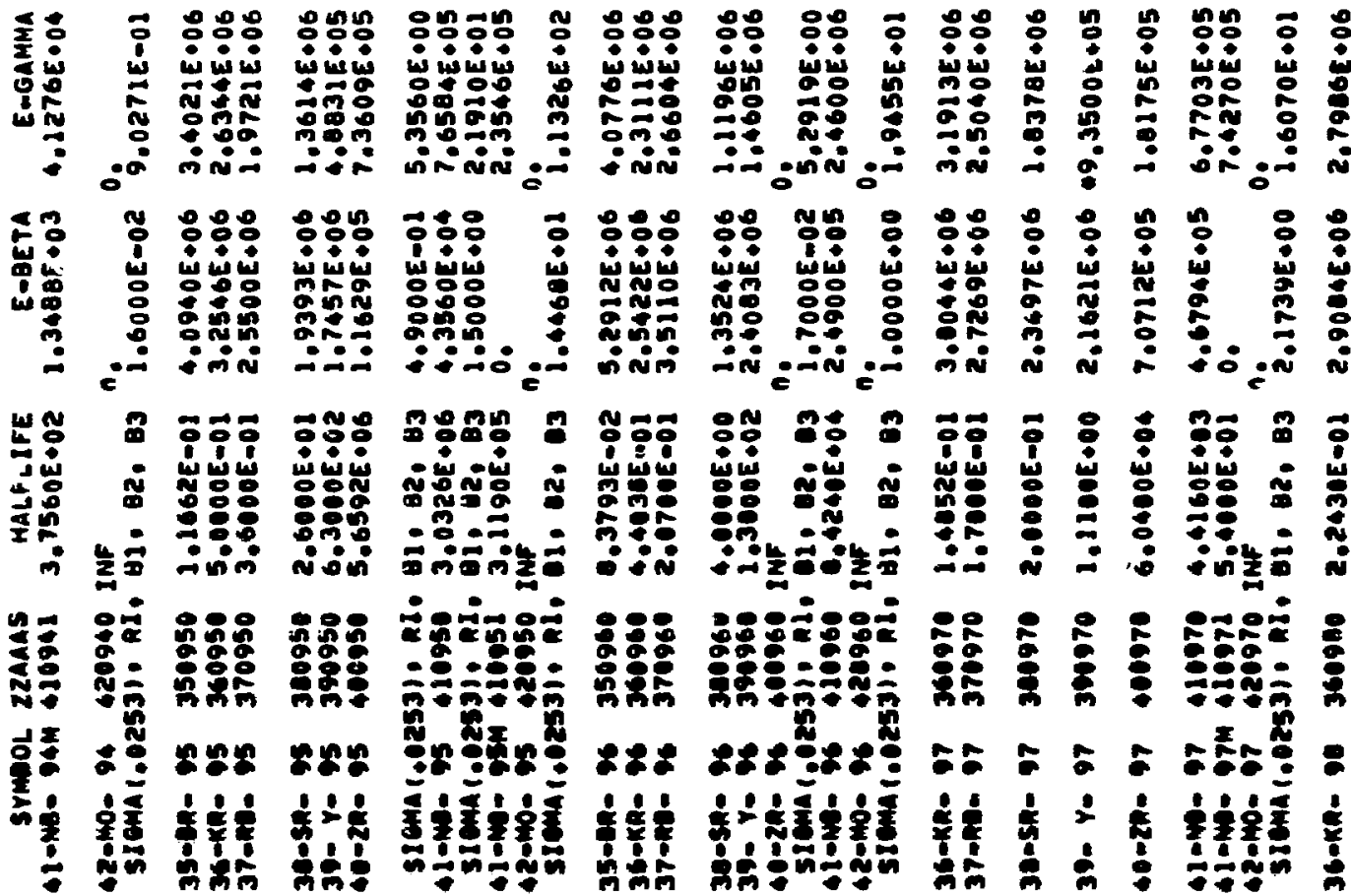




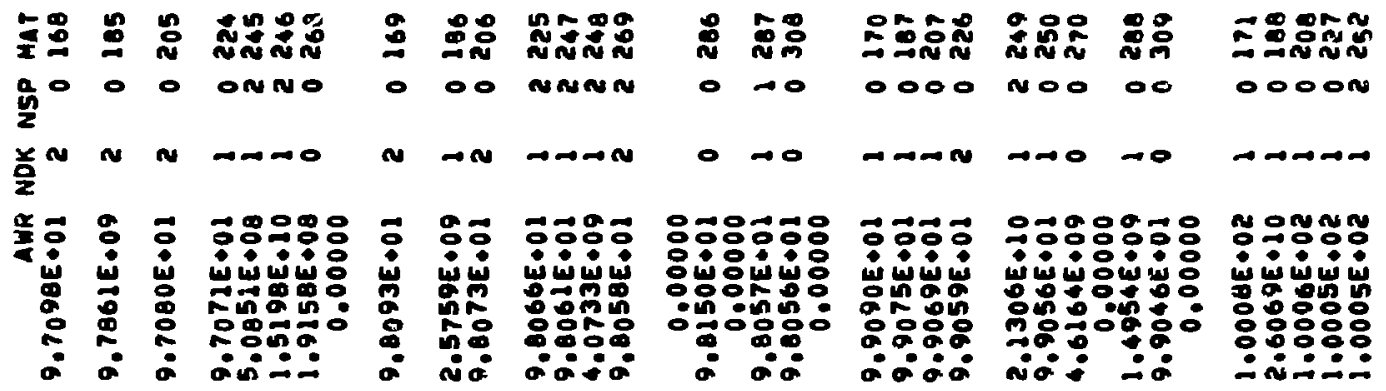

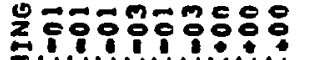

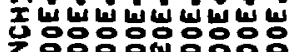

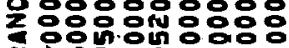
a $\$ 006000$

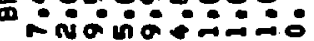

6

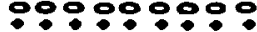

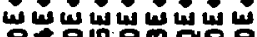

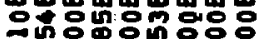

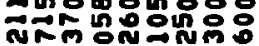

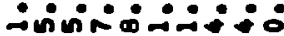

in 00000 otoo

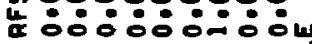
a 00000000 而

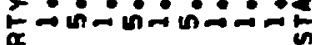

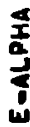

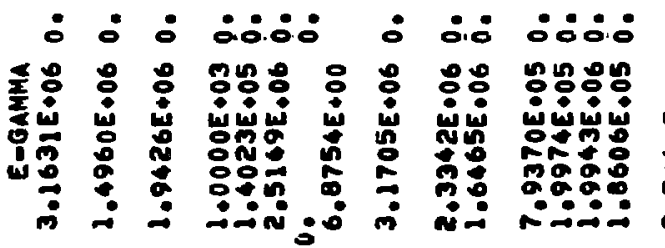

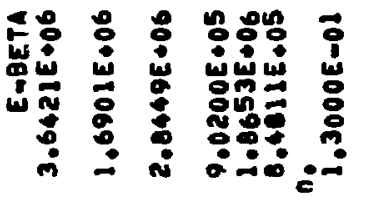

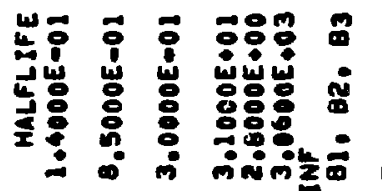

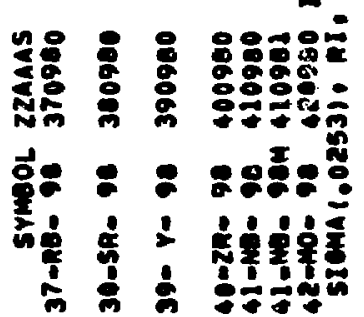

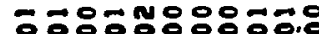

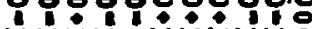

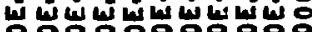

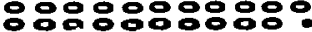
영영영요웅 mO

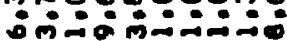

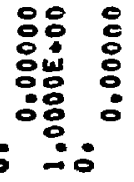

19090900090

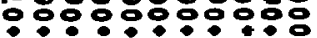
أس

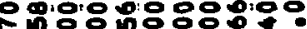

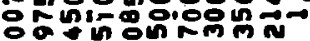
$\because \therefore$ i

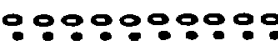

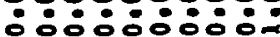

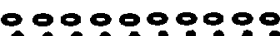

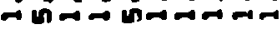
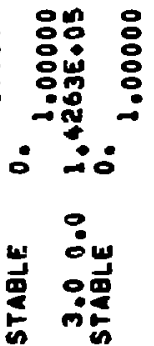

동옹웅후은 $48 \%$

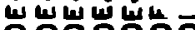

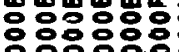

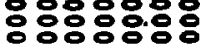
isinis:$$
\text { 용 }
$$

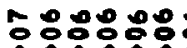

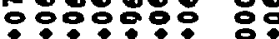
山以山w山 o.0.

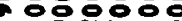

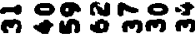

옹ㅇㅇ옹 :5 :

둥웅ㅇㅇㅇㅇㅇㅇ . 는부 $\rightarrow$ 웅요 영요 m $\rightarrow$ 800989.

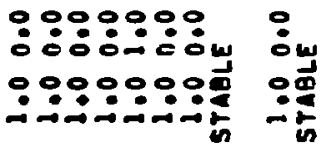
영: 웅잉 $\rightarrow \infty \rightarrow-$ 


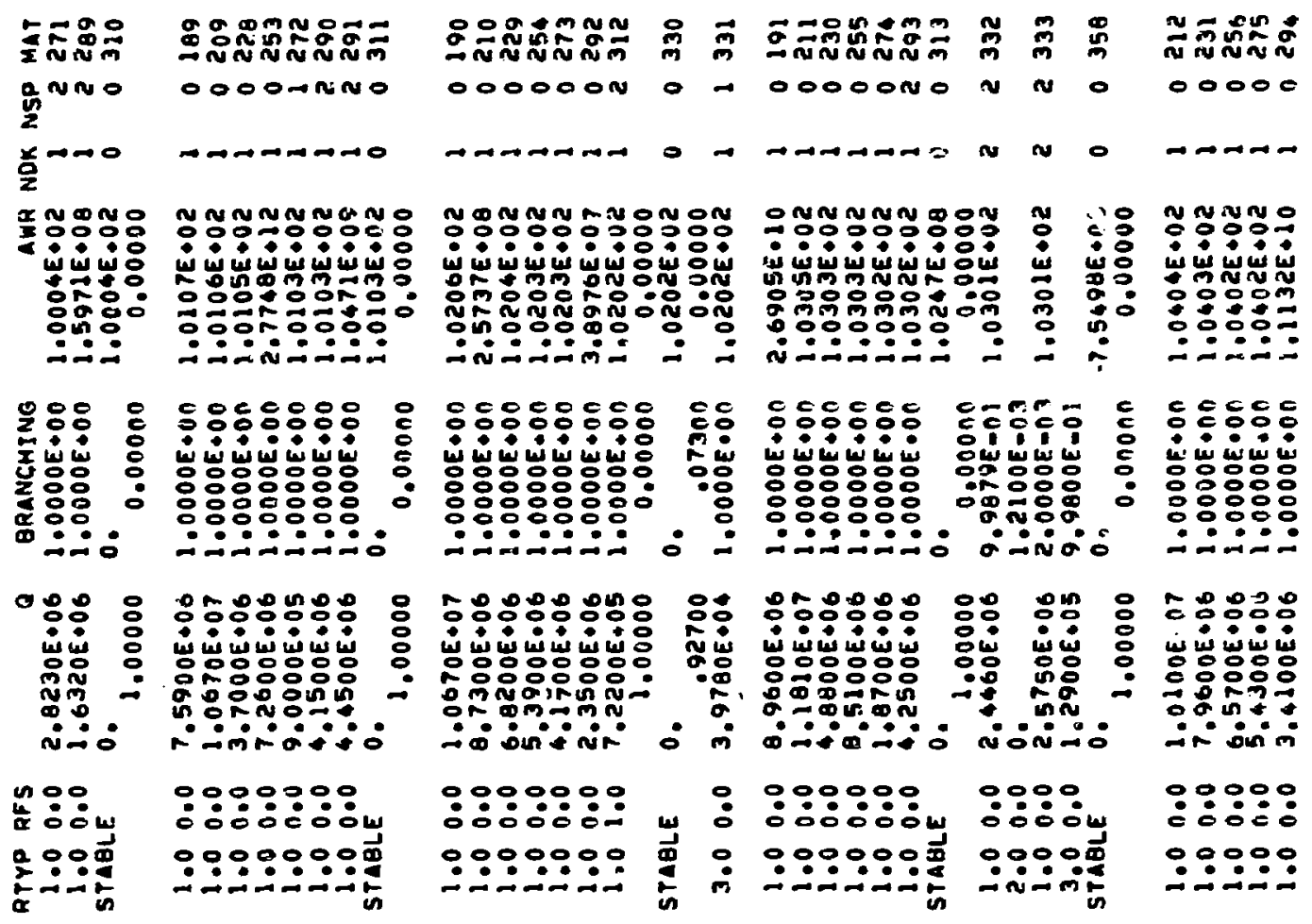

bَ

வீ: வீட்டீடீட்

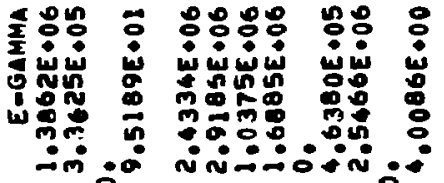

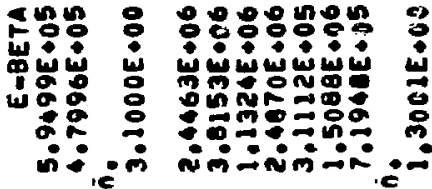

WNN $O$ AีAONON 4:8 9 웅요융

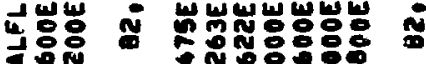
780 $\therefore \frac{1}{2}=$

no유

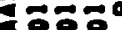
송ํำ웅

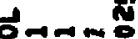
A 80 m - iniminiza 우우우주웅 영응

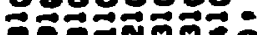

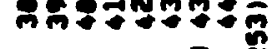

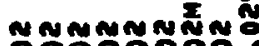

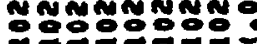
197975

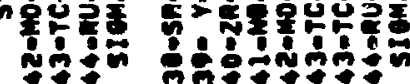

$\therefore$ வ0்0்: : :

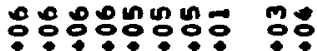

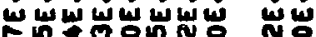

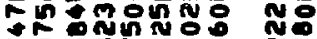
的 mn: :0:

\section{8\%}

융ㅇㅇㅇㅇㅇㅇㅇㅇㅇㅇ w wuw ww 西

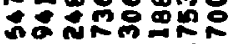
mñ⿻肀二丿

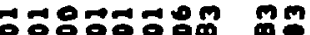
:ㅇํ요

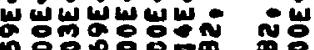


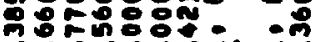

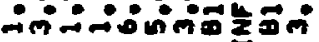
웅ㅇㅇ요두울 (1)

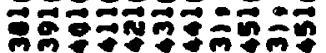

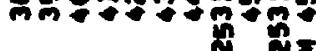

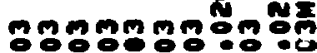
웅우웅웅은

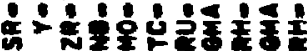

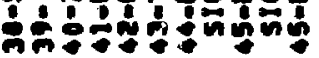

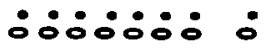

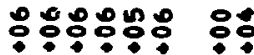
u山w w w w

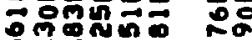
녀요 N miniv: 영유융유 훙요 山w山出 w

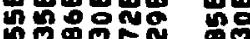
으웡

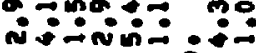
훙요영 숫 씅요용 ¿요. -4m-10:0000000용. 영영영영영

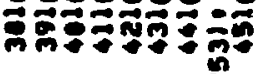
훙ㅎㅇㅎㅎㅎㅎㅎㅎㅎㅎㅇㅎㅇ 웅우웅우우웅

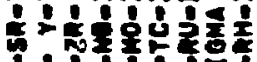
thot?

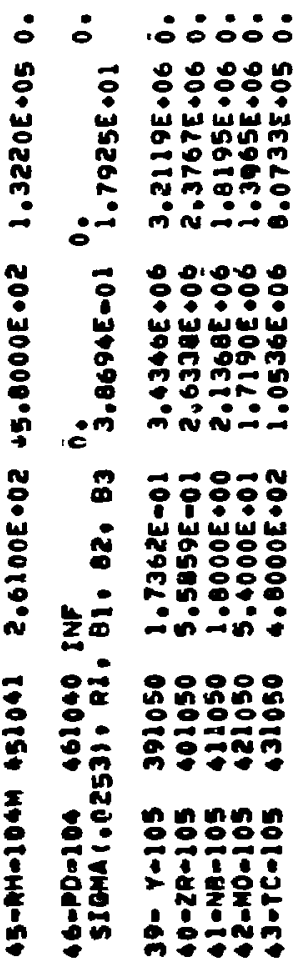




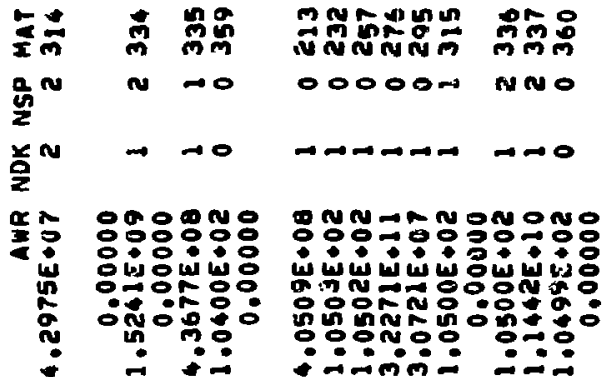

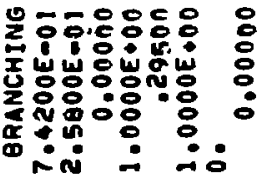

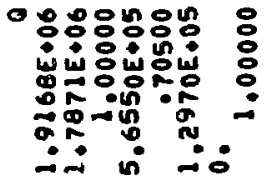

$\begin{array}{lll}n 0 & 0 \\ 4 & 0 & 0 \\ 0 & 0 \\ 2 & 0\end{array}$

交

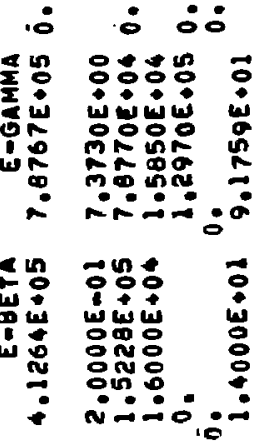

世요요요 m

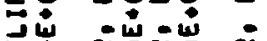

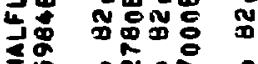

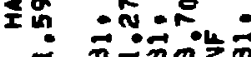

- $3-3 m \geq \infty$

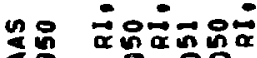

$\sum 0$ 용ํㅇㅇ.

Nै

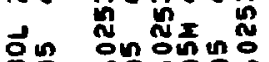

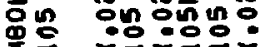

站帝

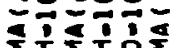

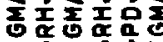

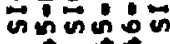

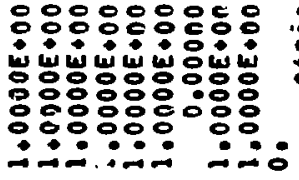

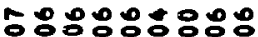
$\div \% 8 \%$

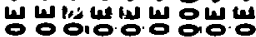

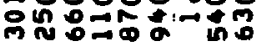
ก -00min mio

00000000

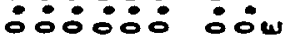
:0909ㅇㅇㅛ

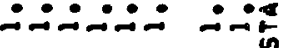

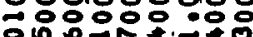

ல்0ல0: 유유유

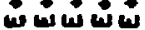

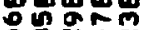

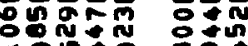
- 냉요 incio:

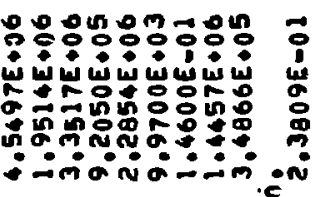

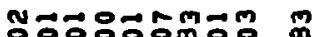
우융ㅇㅇㅇㅠ

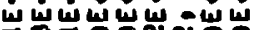

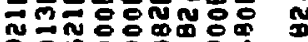
齐出 웅요

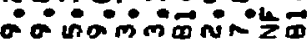
0

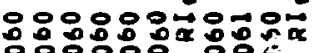

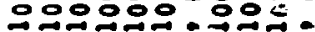

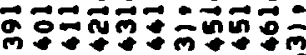
品 恐

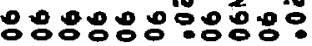

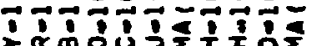

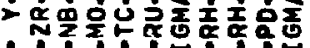

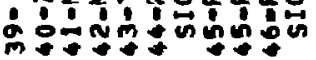

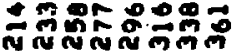

în

00000NNo 00

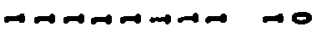

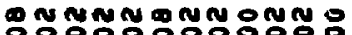

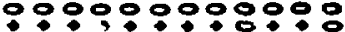

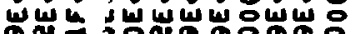

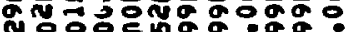

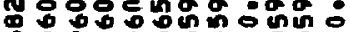

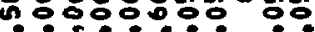

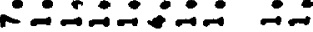

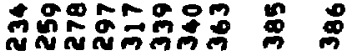

0000nNuo 0 .

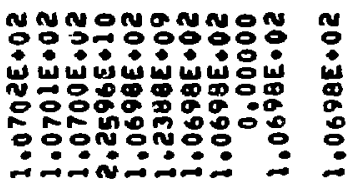

.

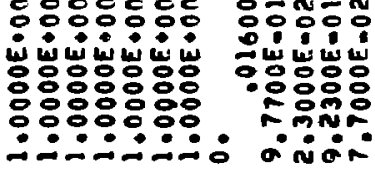
둥유융요 $4+4.98$

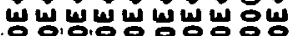

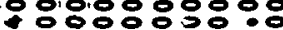

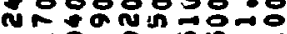
$\because \because \div \div 54$ $\stackrel{8}{\circ}$

0 -0000 웅요웅 웅ㅇㅇ

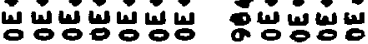
융요웅요

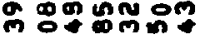
$\because \because \because \because \div$ 0000 40

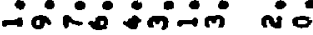

0000000

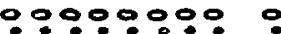
$\therefore: \div 0: 80: 0$ 00000000 on $\because \therefore: \div \div \div \div: \quad=$

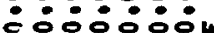
0000000 而 $\because: 90: 0 \%: 4$ $\because \bullet$

0000

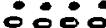
0000 inivi:

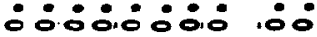
웅ㅇㅇㅇㅇㅇㅇㅇㅇㅇ유융

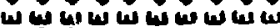
on

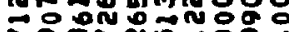

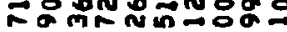
in iñnimi:

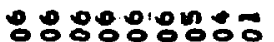
융ㅇㅇ유유유.

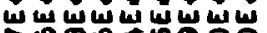
10

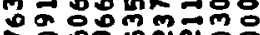
ONNNOO

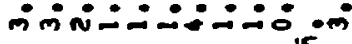

무융유유융ㅇㅇ

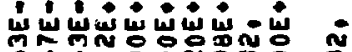

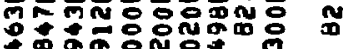

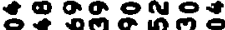

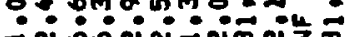
- i : ORORPRORA

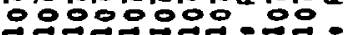

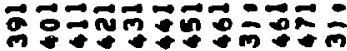

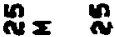
n N M M M O O

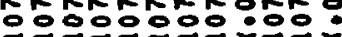

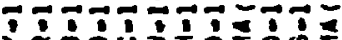

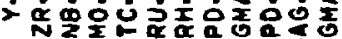

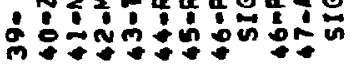

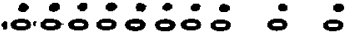

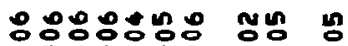

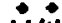
Ny wow w

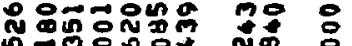

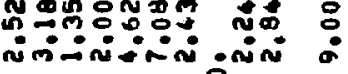

0000un -u

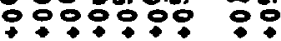
山 융응ㅇㅇ용

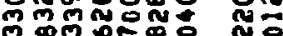
im $=n+\infty \quad \div$ in पic

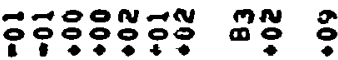

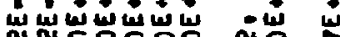
넝등ㅇㅇㅇㅇㅇㅇㅇ 웅 웅

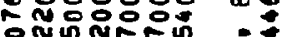
o 0000 000 \%

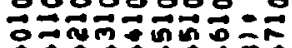
$+2+490+m \alpha$ 况至

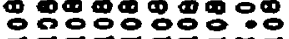

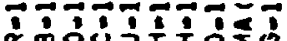

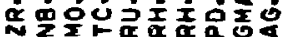

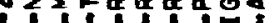

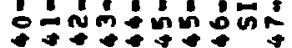




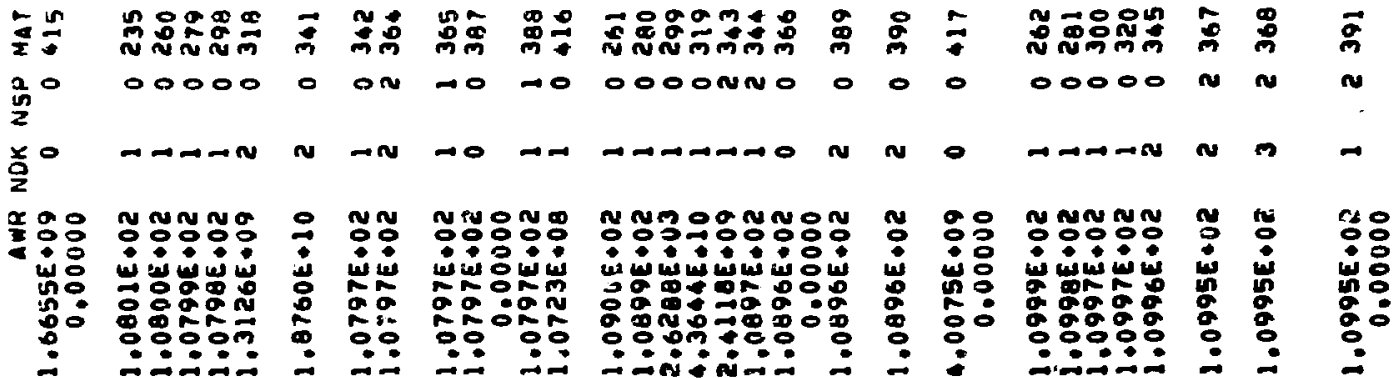

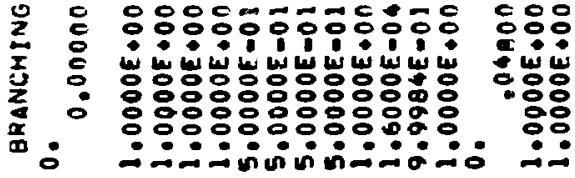

0

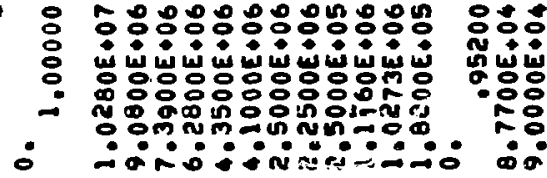

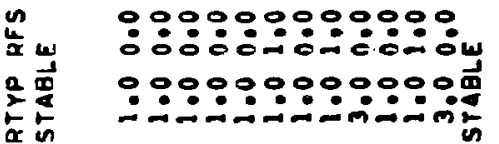

0요

$\therefore$

90

in
등 둥융ㅇㅇㅇㅇㅇ

山山山山山

웅용요용

용용ㅇㅇㅇㅇㅇ

ㅇㅇㅇㅇㅇㅇ.

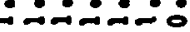

ก

웅우운

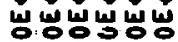

응으응

aina웅

insinis:

$0 \rightarrow m-n$ 웅ㅇㅇㅇㅛ 0 u山w 용ㅇㅇㅇㅛ 융유. ํㅜㅇ:

웅ํㅛㅇㅛ 우운웅 녕 융요용 유: i⿻i土i:

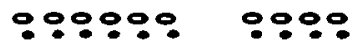
0:0:00月 $000000 \overrightarrow{\mathrm{m}}$ 8000 0000

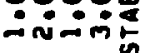

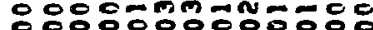

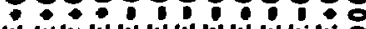

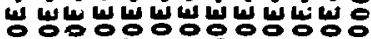

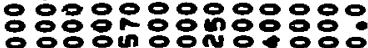

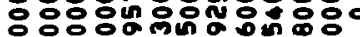

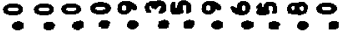

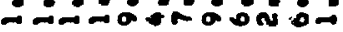

$\vec{a}$

is

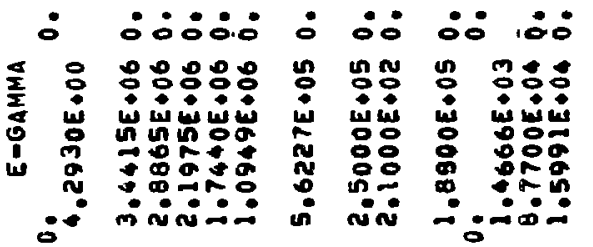

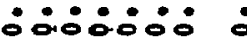
영영영 웅 in is vis w. - ONoR 00

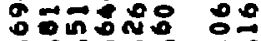
inivion:

:

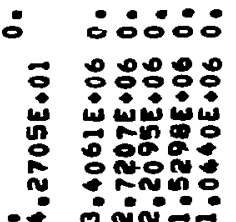

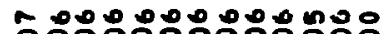
-

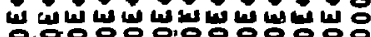

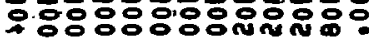

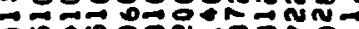
On modn - onis mininivi:

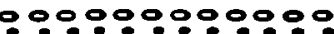

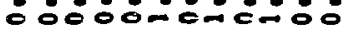
웅ㅇㅇㅇㅇㅇㅇㅇㅇㅇㅇㅡ.

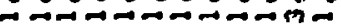

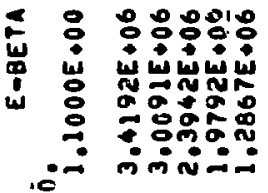

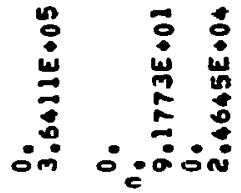

붕ㅇㅇ웅

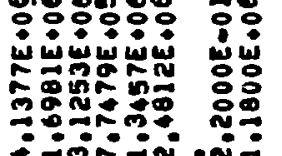

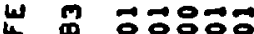

$\rightarrow \sim * N \quad m \rightarrow n$

\& $m: 0$

-

$$
8
$$

$\overrightarrow{0}$

is

b

。

$\because$

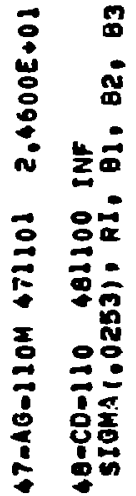

点

눈

崖 $\vec{D}$ -

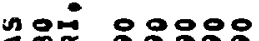

녕중 영영영

我要向

1.

808

$99=$

安家

它

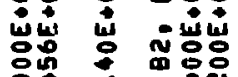

용

n.

-0 00 -0

웅 영형

\%

$20=02$

$9=\frac{1}{8}=$

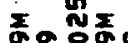

영영

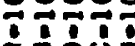

육울웅

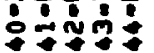

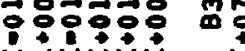

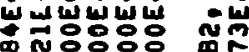

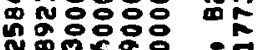

영요

象。

응영으웅ㅇㅇㅇ

$\Rightarrow \rightarrow 007000$

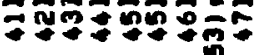

웅ㅇㅇㅇㅎㅇ웡엉

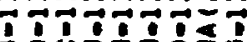

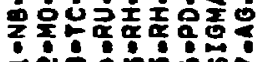

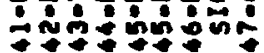

:

$\circ$

훙

wi

용

응

08

กับ

no

98

岁象

응

요용

min

$\operatorname{mo}$

8

w.

똥

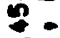

$\therefore \frac{10}{0}$

- ํำ

- 0000

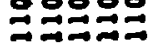

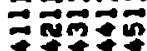

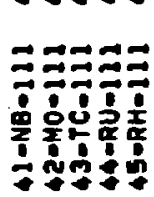




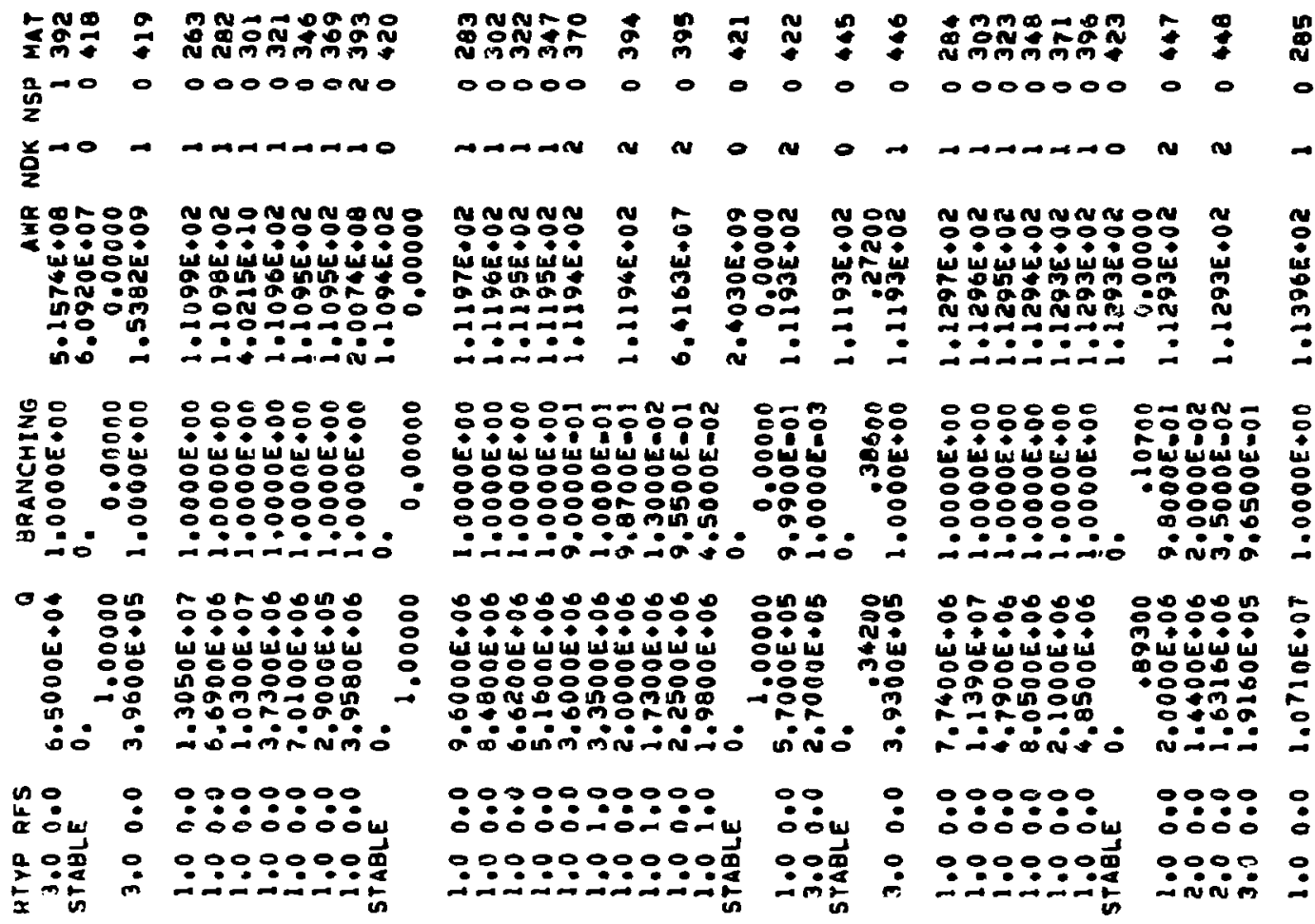

a

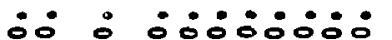

远

它

양

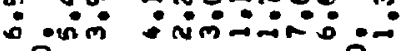

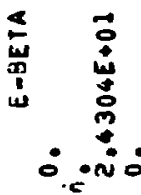

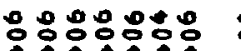

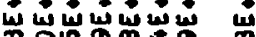

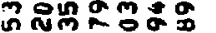

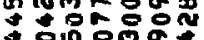

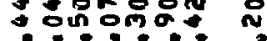

$\rightarrow m$

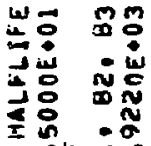

: $\frac{1}{2} \div$

$n \rightarrow 0=-$

$\lim _{x \rightarrow 0}=$

V $\overrightarrow{0}=\overrightarrow{0}$

$N+\infty$

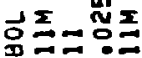

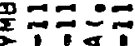

in

官品: N $\rightarrow-170+\div$ 웅ㅇㅇㅇㅇㅇㅛ

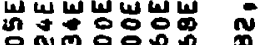

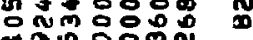

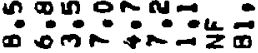

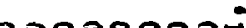

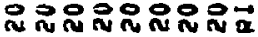
$\Rightarrow=\vec{a}=\overrightarrow{7}=\overrightarrow{7}=$ $\rightarrow$ तैm ง บบNบㅗㅇ $\overrightarrow{1} \overrightarrow{0} \overrightarrow{0} \overrightarrow{0} \overrightarrow{1}=$

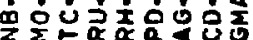

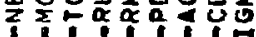

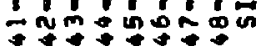

$\therefore 0: 0:$ 웅ㅇㅇㅇㅇㅜ .

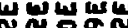
$\circ \mathbb{8}$ 4용 in $\rightarrow: 0$

09890 的的的 $0=07$

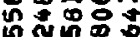
$\rightarrow$ Nom in Niv-

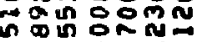

숭ㅇㅇㅇㅇㅇㅇ 山 س إن งก บี่ ㅇํㅇㅇㅇㅇ o un inio:

으요요요

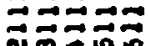
부용 $m m m g$ $\because \div \overrightarrow{1}=0$

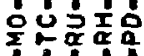
$5 \div \% \frac{\pi}{0}$ ำ:

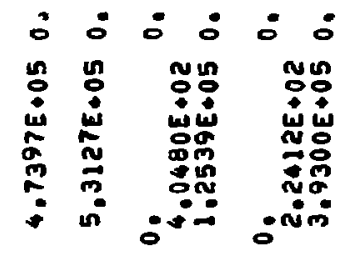

ல்ல்லீல் : 웅ㅇํㅇㅎㅇ 훙으 is is m ติก

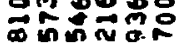
in

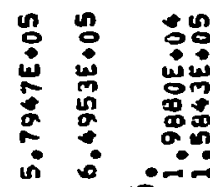

0
$\vdots$
0
0
0

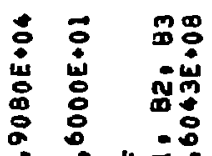

$\sum_{1}^{4}$

웅영영ㅇㅇ 훙응

山 س

mรN年0

ดั จNํำ

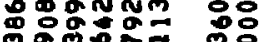

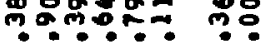

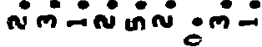

题

บi

\$o

- :

늘

뭉휴융영요

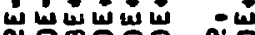

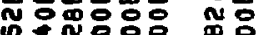

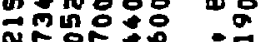
구운

요 $\vec{m} \ddot{a} \vec{m}$

番离

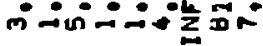

웅ㅇㅇ우웅

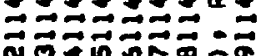

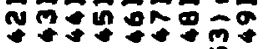

$2 \pm \pm \pm \pm \pm \pm \stackrel{0}{0}$

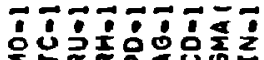

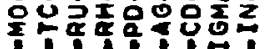

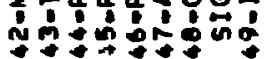

量

$\stackrel{3}{:}$

용

$\div$ .

? 


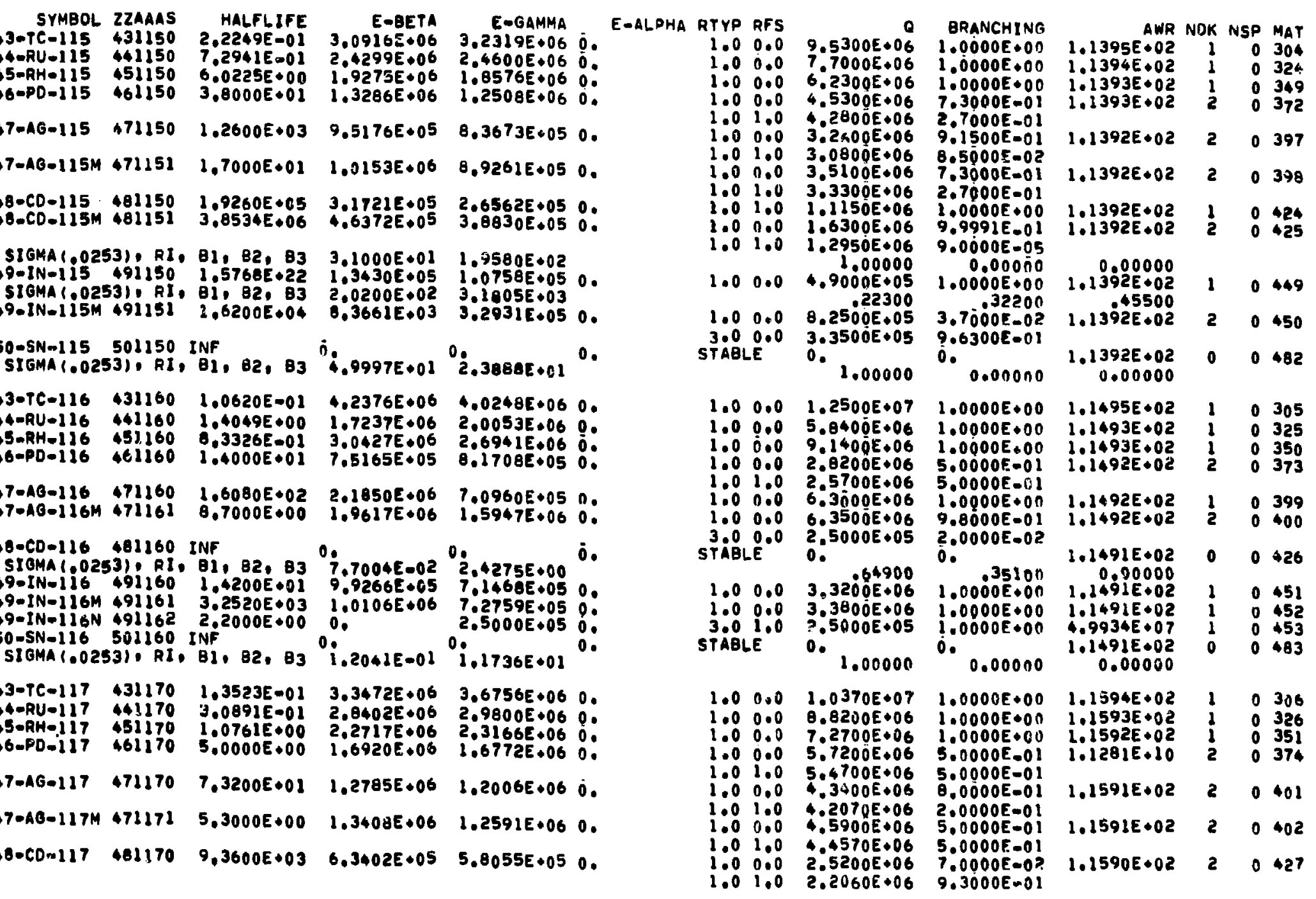




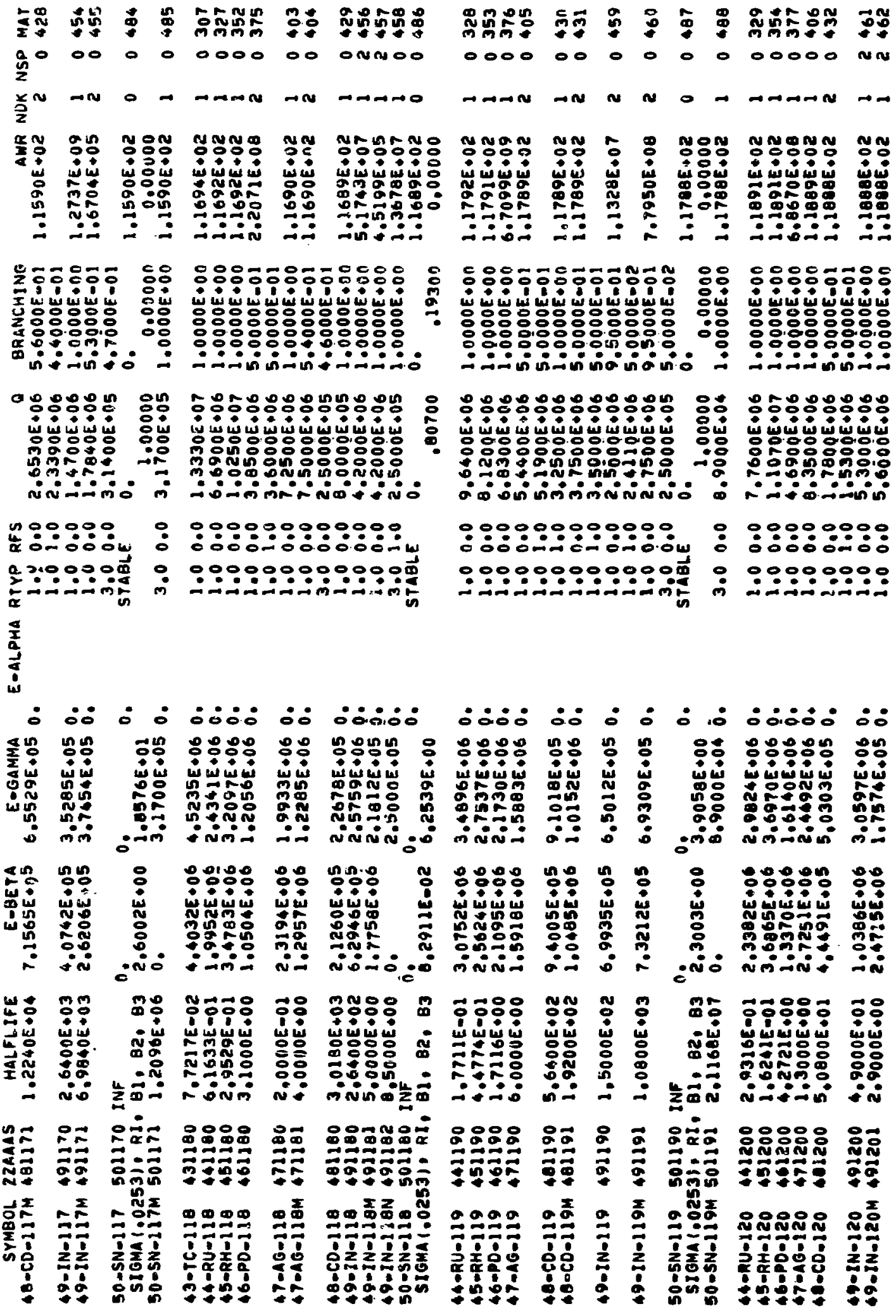




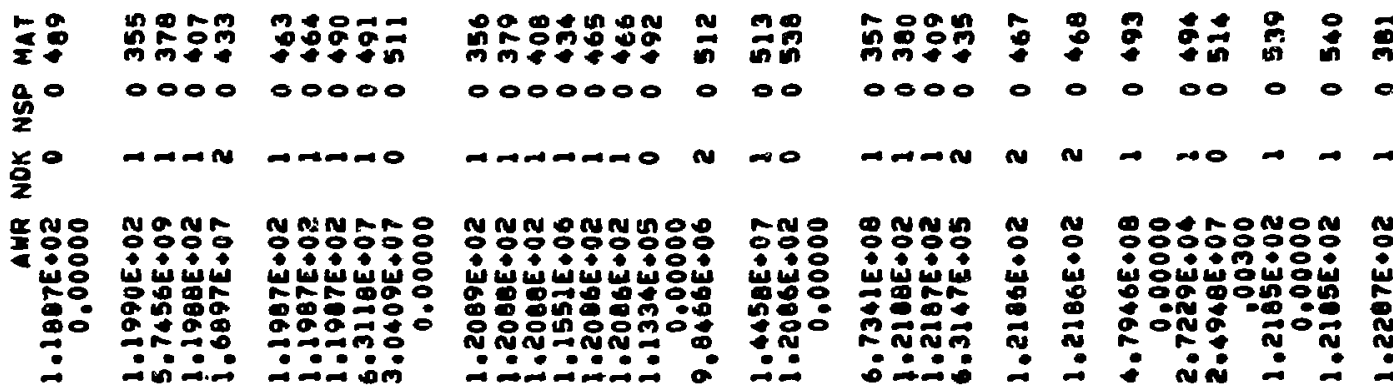

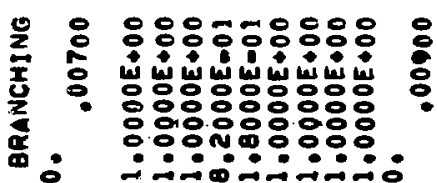

- $\stackrel{8}{\circ}$

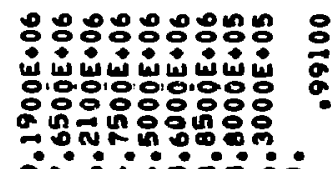

$0 \quad 0+5+\min 0$

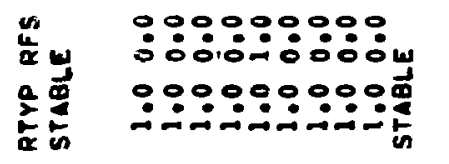

号

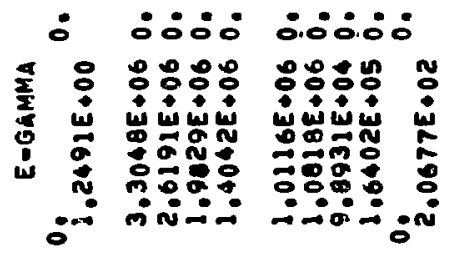

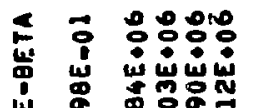
ㅇํ능

\& No

in in:-

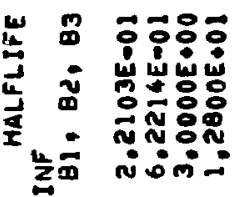
웅ㅇㅇㅇ u山w w

유유요 욤

ภ유유 $\because-1: \div 0$

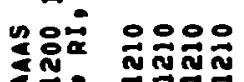

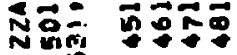

点

ㅇㅇㅇㅠ

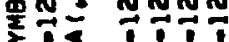

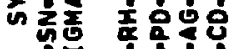

品
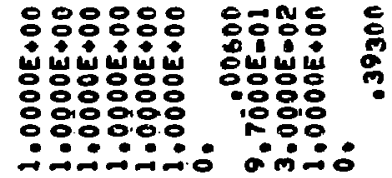

ㄷㅇㅇ유유유

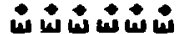

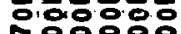

우웅요

$\because 70 \%$

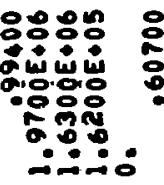

000090 - 00.064

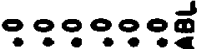

090

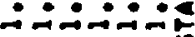
0003 $\rightarrow 00$

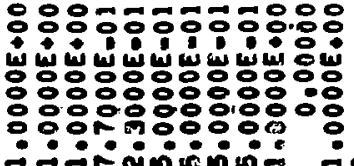

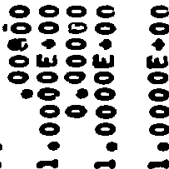

동유융융유융ㅇㅇㅇ

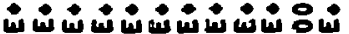

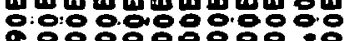
웅요웅요운

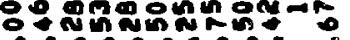

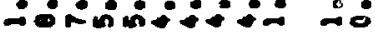

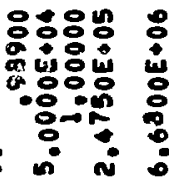

0000000000 0 0 .

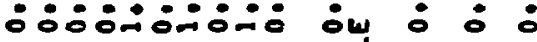

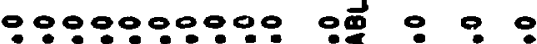

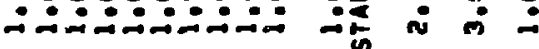

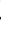

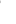

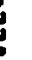




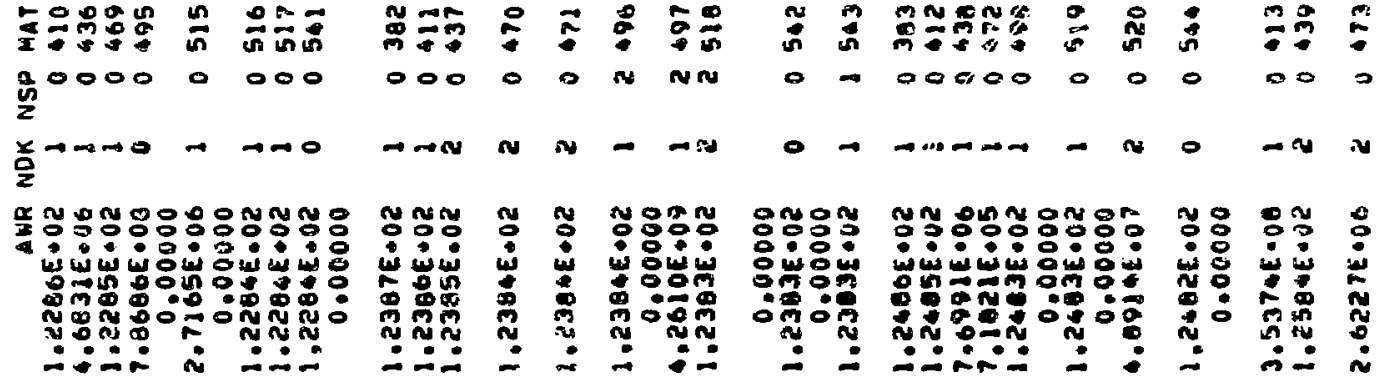

능ㅇㅇ 드응요

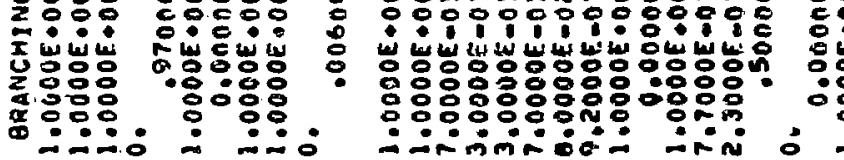
둥응동응ㄷㅇㅇㅇㅇㅇ unw

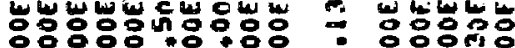

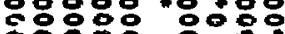

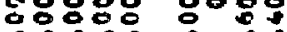

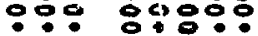

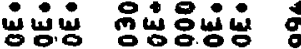
ㅇㅇㅇ

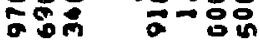
ini: i :No

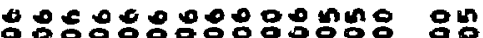

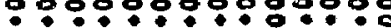

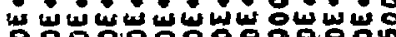

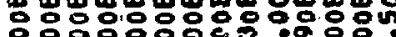

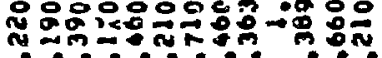

$8:$

용 $-9$ - Dobriusin nis: 0 -

$0+0 \%+9909 n$

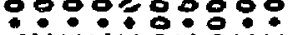
山山ش山ش

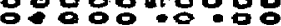

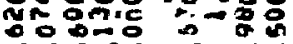
영영요웅

유:유: : :

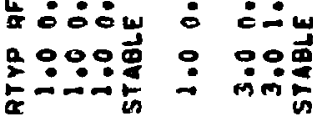

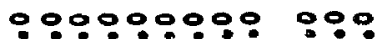

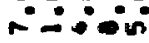

m min

जis:

$\frac{1}{3}$
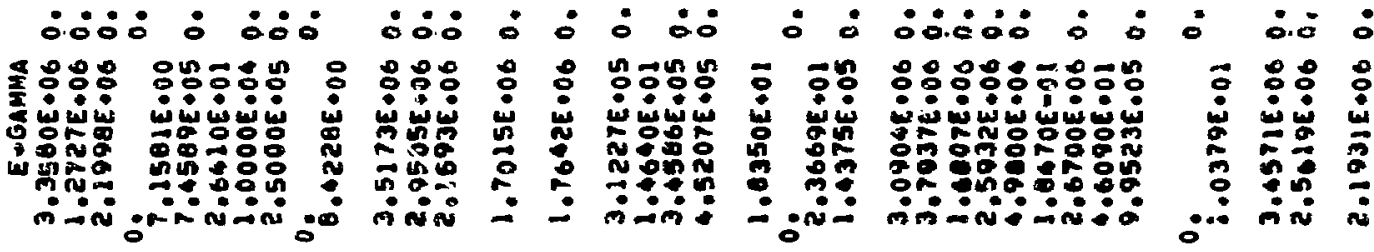

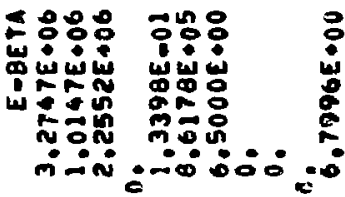
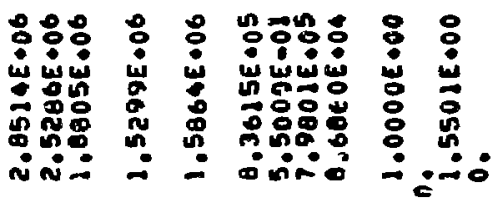

웅ㅇㅇ웅ㅁㅇㅇㅛ

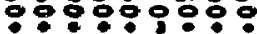
ش م=NOOOOOO

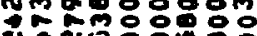

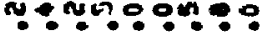
um invimina

눙웅 형영요

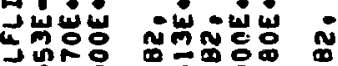
†뽕

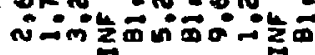

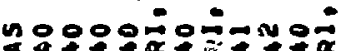

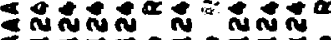

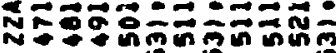
N

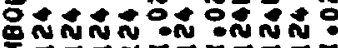

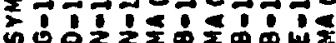

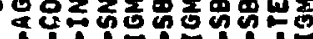

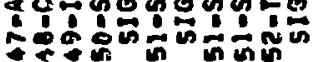

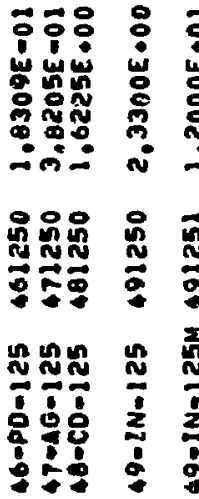

แำํำ iv

$m m$

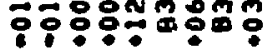

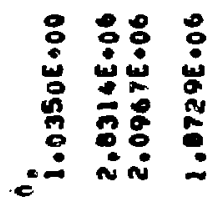

수

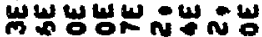

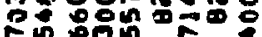

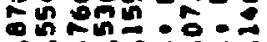

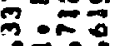

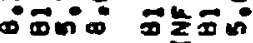

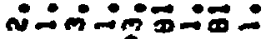
- $0 \div 0$ $00900=0$ 웅

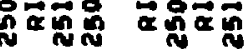

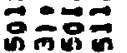
ํㅜㅇํำ ํํำ 色

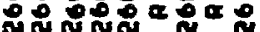

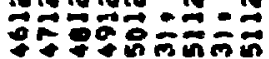

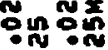
둥: 공ㅎㅇ용 is

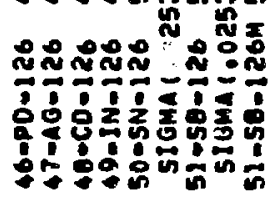

00090 -

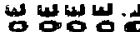
용요유 $\because \div 0 \%$ $-20$

9900 $00-00$ :요웅

요용

禹

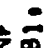
$\omega$ 둥

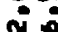

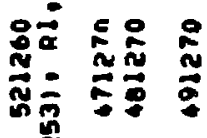

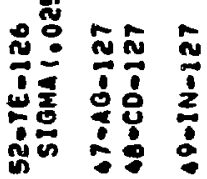




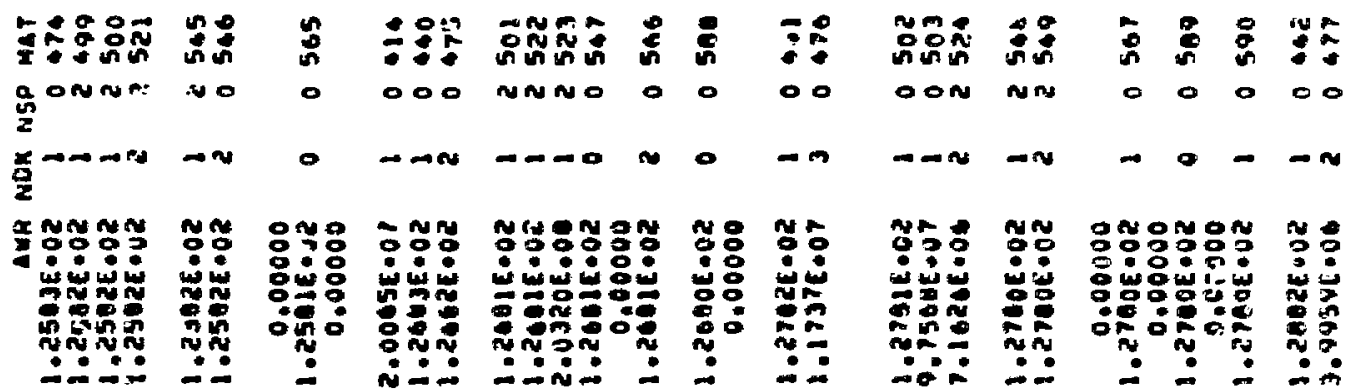

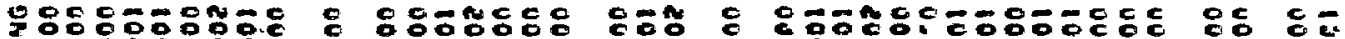

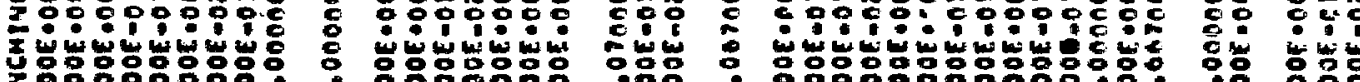

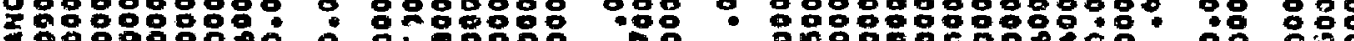

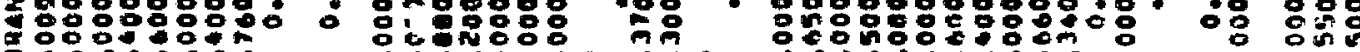
:DE:No

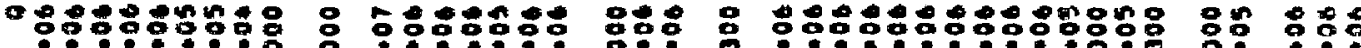

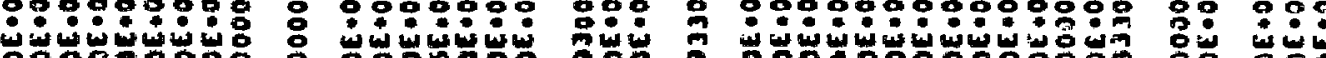

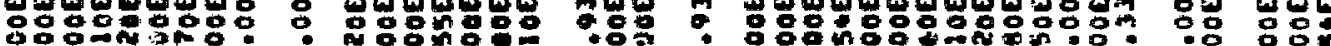

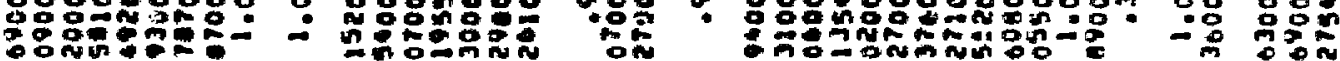
Onm-

no9.9090 \% $2909090 \%: 9$

$\sum^{2}$
9:90999 $000=0$. $0000000 \%$ $--\infty-2=2 \leq n \leq$

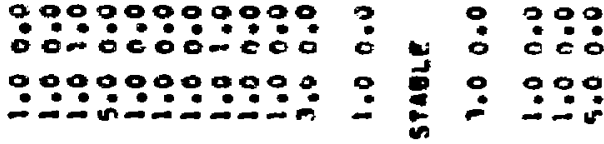

$\frac{1}{2}$

$+0^{\circ}: \div$

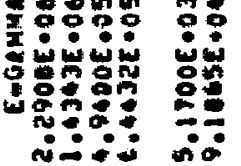

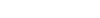

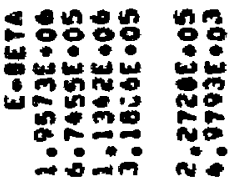

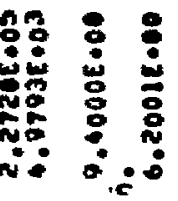

48워요

-

3 8R

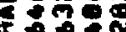

inim

क $=0=0$

\& 0 n 수우응

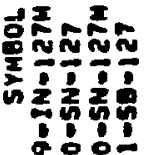

80

$\boldsymbol{0} \infty$

눙 i

mo:

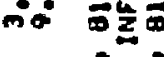

$g=\div 8=$

in

N

กั่

종

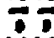

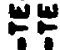

$=2 \div$

운

요요

슨

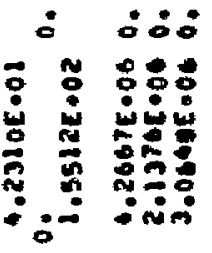

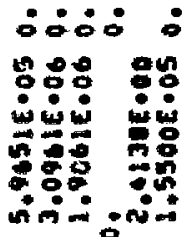

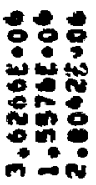

요 2

년

읍 눙요

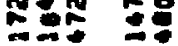

n:0

$-60$

우웅

15 똥

808

260

$\therefore-1$

2요요

녕영

892

뚠

in:

웅

ती

o 00000

요룰

489

오웅

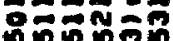

nต $\operatorname{con}$

$\div 2 \div$

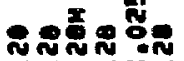

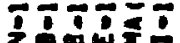

26후웅

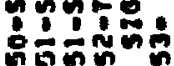

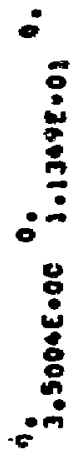

:.

88

4

8

8?

in

88

훙

푸

$+8$

in

8

8웅

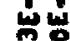

\%:

$\div 8$

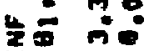

$\div \frac{0}{6} 8$

웅웅

옹

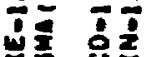

- 0 92

$\div \div 8$

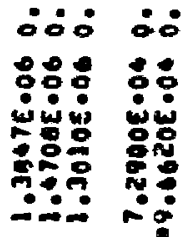

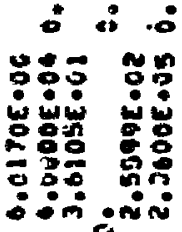

:0

06

no

898 8

nW

$42=$

1웅

$\because-\infty$

웅용

붕형

옹

요

:0:

항뭉

\%ㅇ

in

8후웅

898

4⿻川

080

920

$\because 8$

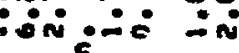

80

$m+m s n$

80

눙

ํㅗ N옹

$-0.0 \%$

89

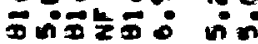

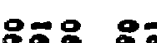

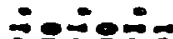

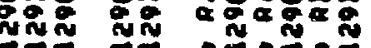

88

분

80

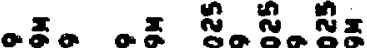

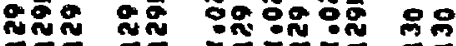

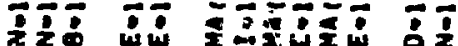

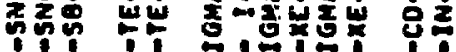

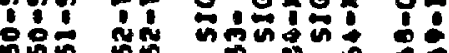




\begin{tabular}{|c|c|c|c|c|}
\hline $\begin{array}{r}S Y \\
-S N=\end{array}$ & $\begin{array}{l}\text { ZZAAAS } \\
\text { S01300 }\end{array}$ & $\begin{array}{r}\text { HALFLIFE } \\
2.2200 E \bullet 02\end{array}$ & 5.0250E & $\begin{array}{r}E=G \\
6.8651\end{array}$ \\
\hline 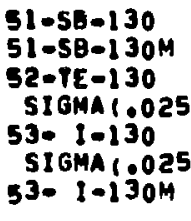 & $\begin{array}{l}511300 \\
511301 \\
521300 \\
531.91 . \\
531300 \\
531.01 . \\
531301\end{array}$ & $\begin{array}{l}3,9600 E \cdot 02 \\
2,22 C 0 E \circ 03 \\
I N F \\
B 1, \forall 2,03 \\
4.4640 E \circ 06 \\
01, \quad B 2,03 \\
5,3,00 E \cdot 02\end{array}$ & $\begin{array}{r}1.2607 E \circ 04 \\
1.0932 E \cdot 00 \\
n, \dot{0} \\
2.0997 E-01 \\
2.9500 E \cdot 05 \\
1.8000 E .01 \\
1.3467 E .05\end{array}$ & $\begin{array}{r}2.1600 E .06 \\
-3.0400 E .06 \\
0.0 \\
3.5360 E-01 \\
2.1200 E .06 \\
1.0100 E .02 \\
3.4705 E .05\end{array}$ \\
\hline & & i. & $6.2003 E-00$ & $0.4 .1912 E$ \\
\hline & 10 & $\begin{array}{l}1.1926 E-01 \\
3.0000 E-01\end{array}$ & & \\
\hline & & & & \\
\hline & & $\begin{array}{l}1.5000 E \bullet 03 \\
1.0000 E \bullet 05\end{array}$ & & \\
\hline $53-1-131$ & 531310 & $6,9474 E \cdot 05$ & $1.8550 E \cdot 05$ & $3,8928 E \bullet C$ \\
\hline $\begin{array}{l}\text { S4-XE-I } \\
\text { SIGMA? } \\
\text { S4-XE-l }\end{array}$ & $\begin{array}{r}5 \\
53 \\
5\end{array}$ & $\begin{array}{l}2.83 \\
2,83 \\
9 E \cdot 06\end{array}$ & $\begin{array}{l}1.0000 E=01 \\
n: 0.9984 E 001\end{array}$ & $\begin{array}{r}.0150 E \cdot 00 \\
.7856 E \cdot 02\end{array}$ \\
\hline $\begin{array}{l}48-C O-132 \\
49-1 N-132 \\
50-5 N-132 \\
51-5 B-132 \\
51-5 B-132 M \\
52-1 E-132 \\
51 G M A 1.025 \\
53-1-132 \\
54-X E-132 \\
\text { SIGMA } 1.025\end{array}$ & $\begin{array}{l}401320 \\
691320 \\
501320 \\
511320 \\
511321 \\
521320 \\
5311 . R 1 . \\
531320 \\
561320 \\
531 . R 1 .\end{array}$ & $\begin{array}{r}1.4679 E \bullet 01 \\
1.2000 E-01 \\
.0000 E \bullet 01 \\
1.2600 E \bullet 02 \\
2,4000 E \bullet 02 \\
2,8000 E \cdot 05 \\
B 1, \forall 2, B 3 \\
9,2260 E \cdot 03 \\
I N F, \\
\theta 1,02, B 3\end{array}$ & $\begin{array}{r}2.6912 E \bullet 06 \\
3.6247 E \bullet 06 \\
6.6029 E \bullet 05 \\
1.7221 E \bullet 06 \\
1.6955 E \bullet 06 \\
6.0050 E \bullet 04 \\
2.0000 E=03 \\
5.246 E E .05 \\
0.5003 E=01 \\
4.5003 E=01\end{array}$ & $\begin{array}{l}3.9976 E \bullet 06 \\
4.0606 E \cdot 06 \\
1.3228 E \cdot 06 \\
2.0066 E \bullet 06 \\
2.0396 E \bullet 00 \\
2.6860 E \bullet 06 \\
4.9800 E=03 \\
2.2377 E .06 \\
0.06 \\
i .7263 E \cdot 00\end{array}$ \\
\hline & & & 3. & \\
\hline 3 & 511330 & $1,4400 E+02$ & $5.3711 E .05$ & $-2.5000 E .06$ \\
\hline & & $240 E \cdot 03$ & 8.1 & 6 \\
\hline
\end{tabular}

E-ALPHA RTYP QES 1.00 .0 1.01 .0

1.00 .0

1.00 .0 sPagle

1.00 .0

1.00 .0

$\begin{array}{lll}1.0 & 0.0 \\ 3.0 & 0.0\end{array}$

STABLE

$1.00 .0 \quad 1.05505007$ $1.00 .0 \quad 8.3900 E+06$ $5.00 .0 \quad 3.3657 E \cdot 06$

$1.00 .0 \quad 4.6300 E \cdot 06$

$1.00 .0 \quad 3,4000 E+06$

$1.01 .0 \quad 3.2180 E \bullet 06$

$1.0 \quad 0.0 \quad 2.2490 E \cdot 06$

$1.0 \quad 0.0 \quad 2.4310 E+06$

$3.0 \quad 0.0 \quad 1.8240 E \cdot 05$

$1.00 .0 \quad 9.7080 E+05$

$1.01 .07 .7600 E+05$

STABLE

1.00000

0.1 .00000

$\begin{array}{lll}3.0 & 0.0 & 1.6393 E .05\end{array}$

$1.0 \quad 0.0 \quad 7.3800 E .06$

$1.0 \quad 0.0 \quad 1.2310 E \cdot 07$

$1.00 .0 \quad 3.0200 E .06$

$1.0 \quad 0.0 \quad 6.0800 E \cdot 06$

$1.00 .0 \quad 6.0800 E \cdot 06$

1.00 .0

$6.0800 E \cdot 06$
$5.0500 E .05$ .50000

$1.0 \quad 0.0 \quad 3.58000 .06$ STAQLE

$\begin{array}{ll} & \\ 1.0 & 0.0 \\ 1.0 & 0.0 \\ 5.0 & 3.0 \\ 1.0 & 0.0 \\ 1.0 & 1.0 \\ 1.0 & 0.0 \\ 1.0 & 0.0\end{array}$ $0:$

.93500

1.2400E.06

1.0 $1.2594 E+05$

$1.0 \quad 0.0 \quad 2.6160 E+06$

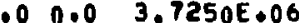

$3.0 \quad 0.0 \quad 3.3450 E+05$
BRANCHING

1.0000E-01 $9.0000 E-01$

$1.0000 E \bullet 100$ $1.0000 E \bullet 00$ 0 .

.08900

$1.0000 E \bullet 00$ 0.00000 $1.5000 E-01$ 0.5000 E.O

$.055 n 0$

$1.0000 E \bullet 00$ $9.0500 E=01$ $9.5000 E-02$

$1,0000 E+00$ $9.3200 E-O Y$

$6.0000 E=02$

$1.0000 E \cdot 00$

Q.2000E-01

$1 . B 000 E=01$

$9.9300 E-01$

$7.0000 E-03$

0. 0.00000

0.00000

$1.0000 E+00$

$1.0000 E .00$

$1.0000 E \bullet 00$

$1.0000 E .00$

$1.0000 E 000$

$1.0000 E \bullet O n$

1.0000E.0D $500 n n$

$1.0000 E \cdot 00$ 0 :

.06500

$1.0000 E 000$ $9.9979 E=01$ 2.2000E-00 $9,7760 E=01$ $2.2400 E=02$ $1.0000 E \cdot 00$ $.7000 E-01$ 1.3000E-01
AER NOK NSP MAT
$1.0063 E+07$

$1.2800 E \cdot 02 \quad 2525$

$\begin{array}{lll}1.20106 .02 & 1 & 2525 \\ 1.2879 E 002 & 5 & 530\end{array}$

1.267 E.02 1058

0.00000

$1.2879 E \cdot 02$

2 0569

$1.2079 E \bullet 02$ Q 0591

0.00000

1.2901E 02

$1.640+E \cdot 06$

10443

1.2979E.02 I 0505

$1.2978 E \cdot 02,2551$

1.2978E.02 2 2552

$1.29786 .02 \quad 2 \quad 2570$

0.00004

$1.2978 E \cdot 02$

0.00000

$1.2978 E .02$

$0 \quad 0592$

1.30815 .02

11593

$1.0992 E .07$

1.30796 .02

$1.3079 E \cdot 02$

$1.3078 E .02$

$1.3077 E .06$

0.00000

1.30776 .02

$1.3077 E \cdot 02$

0.00000

0444

0479

2 506

2520
2529

i 2553

0.00000

I $04 B 0$

$1.3178 E .02 \quad 2 \quad 0507$

$1.3177 E .02 \quad 2 \quad 2530$

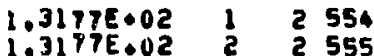




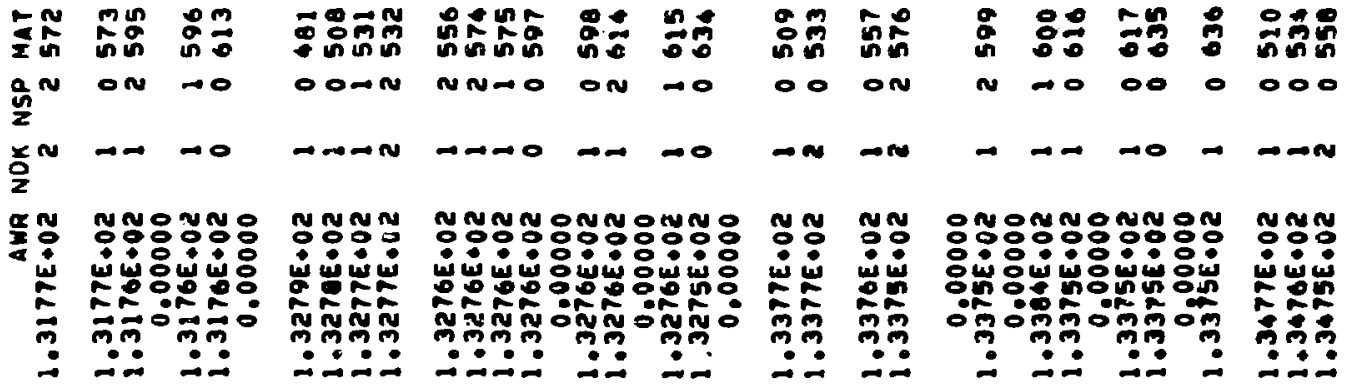

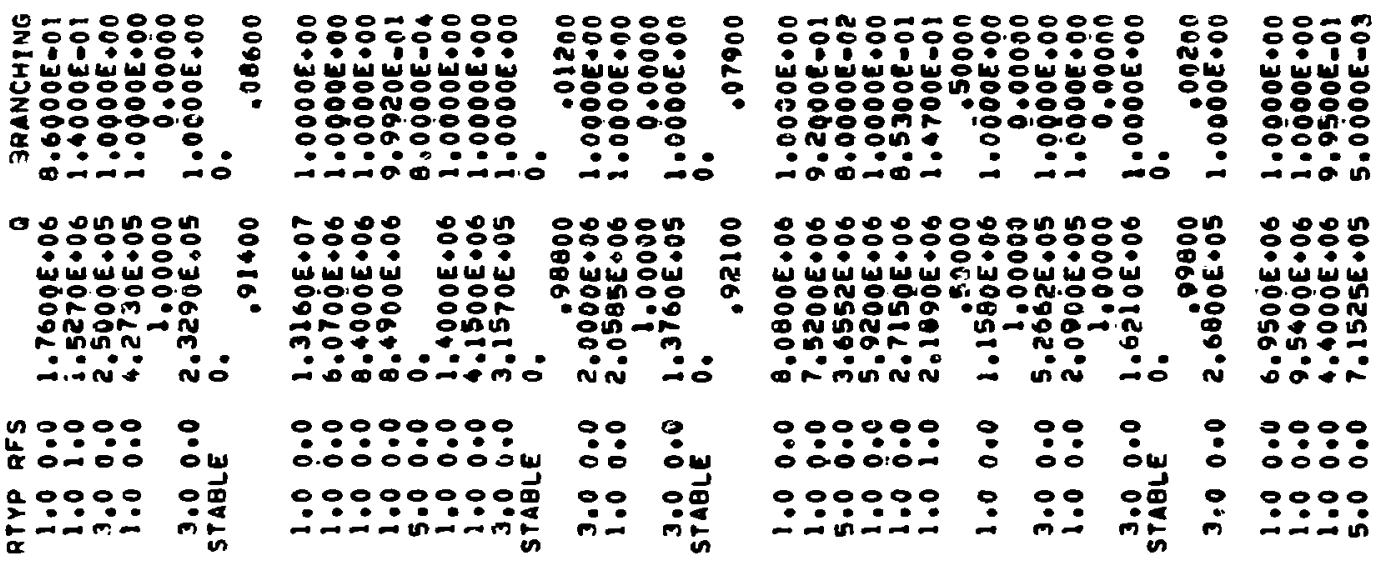

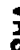

$\frac{a}{a}$

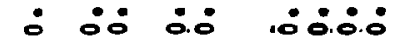

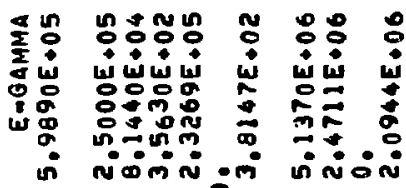

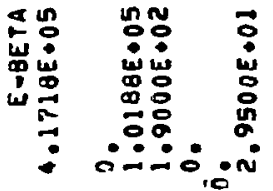

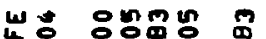

4

14

$\frac{1}{1}$

$\sim$

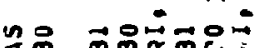

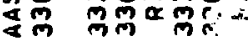

$\sum \frac{\vec{m}}{m}=\overrightarrow{0}$

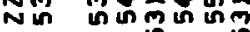

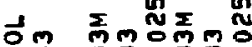

솔 m

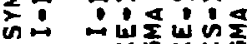

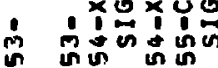

웅용

$8 \%$

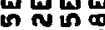

$\Rightarrow: \overrightarrow{\mathrm{n}}$ in

ㅎํㅇํㅇ

- im

우웅

岗岁岁㟧

iิ 0 웅

$\because \div$ in

$\therefore \div:=$

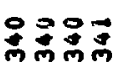

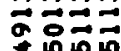

$+ \pm$

mำ

$\sum \sum_{0}^{\infty} \mathbb{N}_{0}^{\infty}$

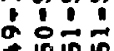

웅요

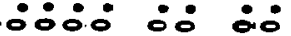

080

ज舟:

\&O

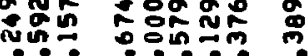

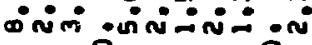

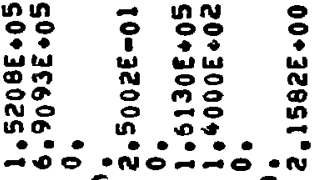
훙영영 연연 $+2$ 용요 in in

: :0 $8:$ i

璐

웅

$\because:$

N $\rightarrow$ 웅웅 m웅후

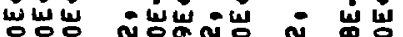
ㅇㅇ

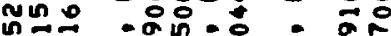

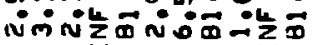

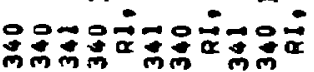
$\vec{\alpha} \vec{m} \vec{m}=\overrightarrow{0} \overrightarrow{\mathbf{n}}=\overrightarrow{0} \overrightarrow{0}$

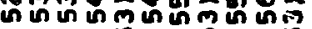

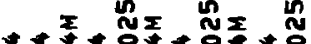

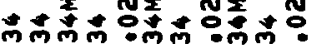
$\because \because \div \div \overline{0} \div \overrightarrow{0}$

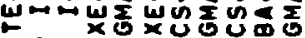

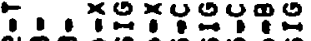

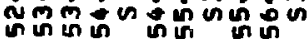

:웅

in

总: m. iv

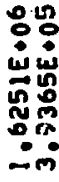

$\overrightarrow{0}$

î

옹응

in

i:

ํำ ำ

92

웅

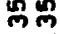

$\vec{N} \vec{B}$

ng

i⿱

운

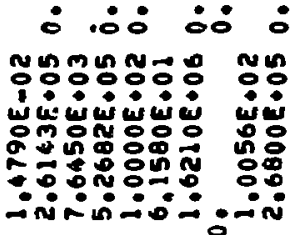

$\therefore: 0$ 800 ivis 0 $\rightarrow \infty$ - 80 im $=$

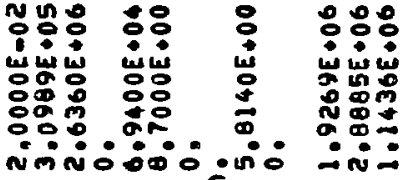

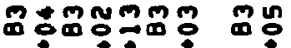

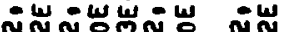
1 0 \% -

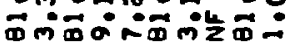

훙ㅎㅇ 山w

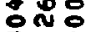
mi - in 우ㅅㅜㅜ

909 임 $\Rightarrow \overrightarrow{0}=\overrightarrow{4}=\overrightarrow{1}=\overrightarrow{0}=\overrightarrow{0}$

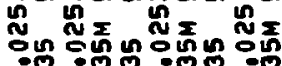
은 nn

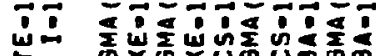

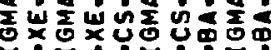
Nm แก แn

กํํำ

$\div \div$ $\sum_{0} \sin$ $8 \div 2$ 은 


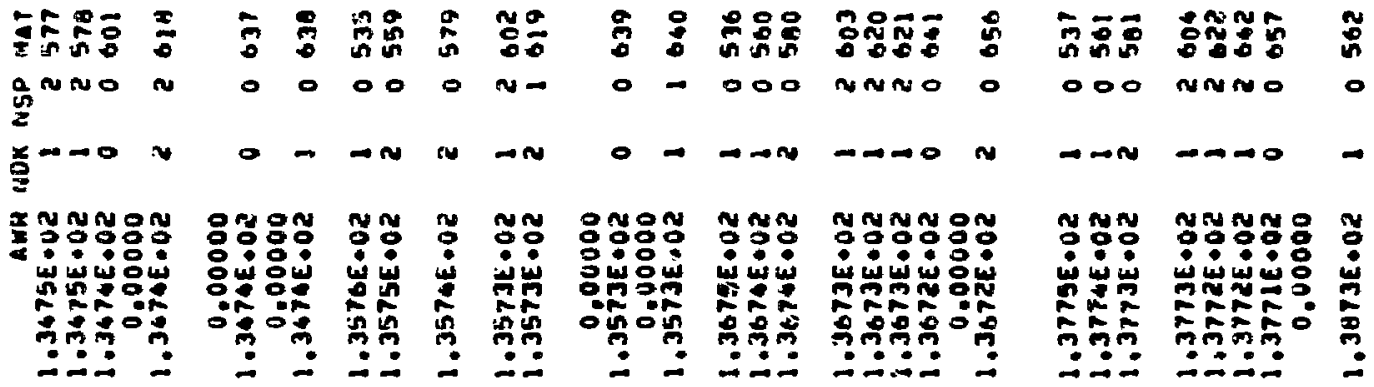

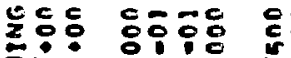

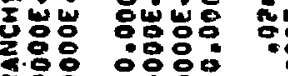 \\ $\div \div 0 \div \div \div$}

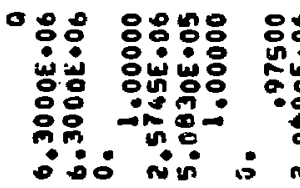

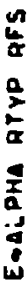

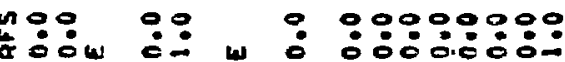

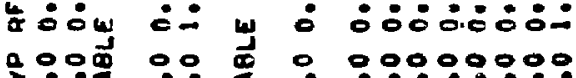

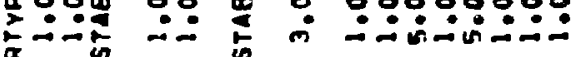
ì

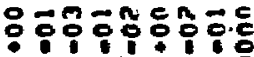

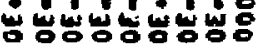

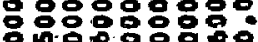

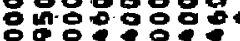
-

옹옹송오옹용웅용

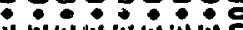

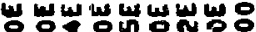

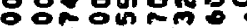
8. Doni-10:

\section{용용

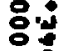 \\ $\rightarrow$}

등영영 도응

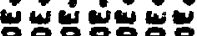

영영영

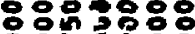

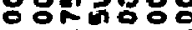

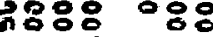

- - on-1: is

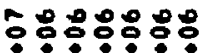

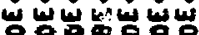

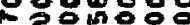

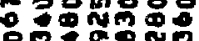

- -

800

8 iw

.8응

$0 \%$

on

- 0000000

:ㅎ:ㅎㅎㄴ

$\because 9$

-0000000

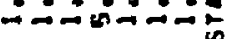

0

0

$\rightarrow$ i

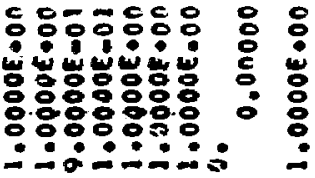

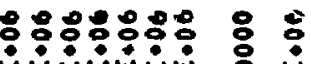

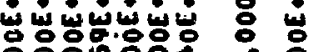

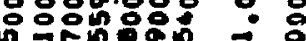

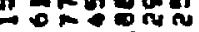

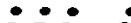

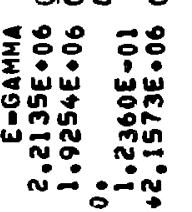

aㅇ $\rightarrow 0$

48: 80

夏舟

i o

๓⿴囗十

$\therefore \therefore:-1$

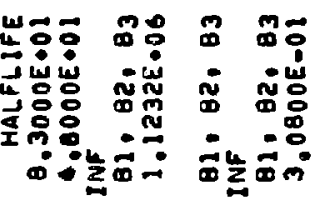

n

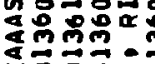

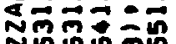

$\therefore=$ रू

ํํํํㅇำ

mmㅜㄴ

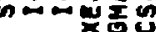

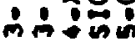

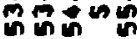

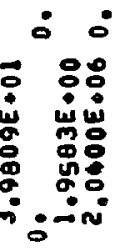

$\rightarrow-\infty=$

ตํำ

N $N=$

이용

$=\frac{1}{1}=$

퐁히응

我品: i:

:

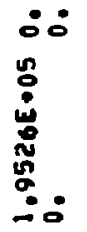

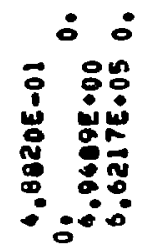

웅웅

응을

$\overrightarrow{8} 8$
0
0
8
8
0
0

急

in

8

$\rightarrow 0$

$8 \%$

w

is

in

in

용

m

$\overrightarrow{0}$
藏出工

ติ

$\because \div \div$

界

$\because \mathbf{1}$ wy

m
:0:

889

48

\%

iv

N.

- ivi

80

un w

क⿻日木

N온

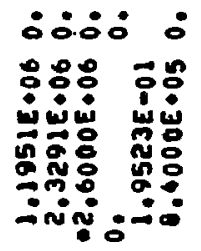

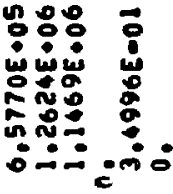

ㅎㅇㅇㅇㅇㅇㅛ

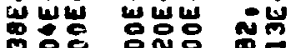

응

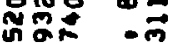

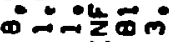

$-\pi 0$

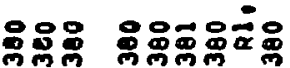

숭

ํํำ

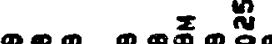

요

$\div \div \div \div \div 5 \div$

号心

in

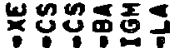

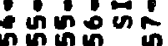

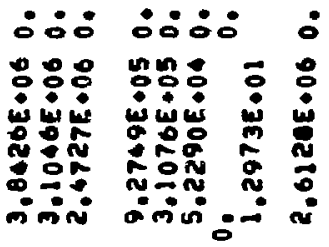

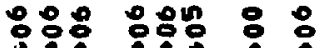

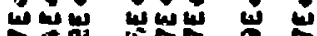

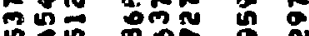

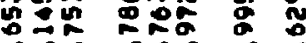

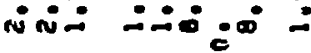

뭉ㅇㅇㅇㅇㅛ

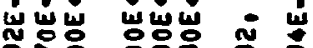
송 웅용 ஸे

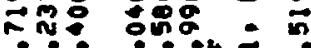
$\therefore-i \quad \therefore \div \frac{2}{2}$

ㅇㅇㅇㅇㅇ우웅

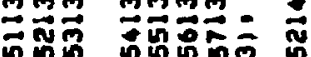

要

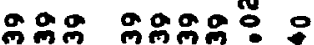

$\because \overrightarrow{2} \div \overrightarrow{8} \div=$

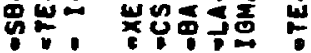

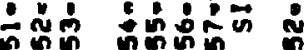




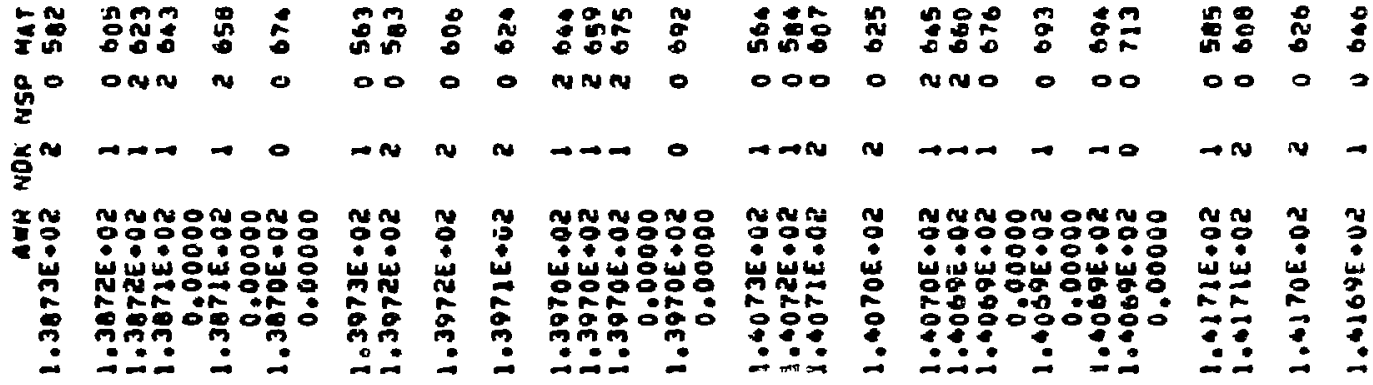
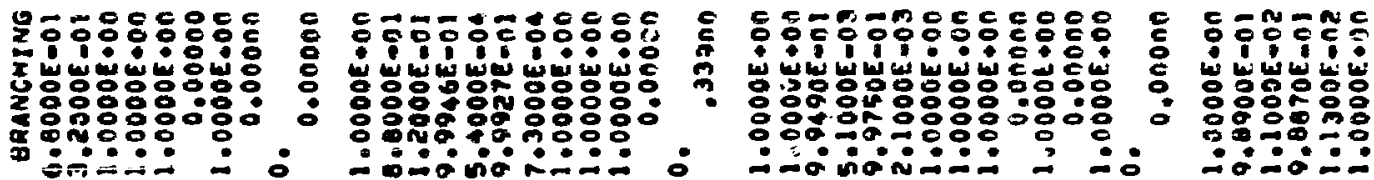

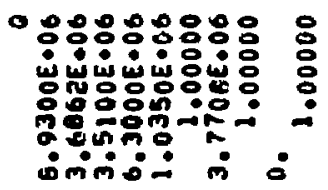

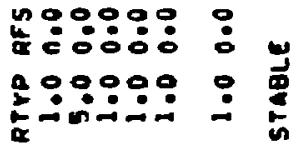

ta

$\frac{1}{8}$

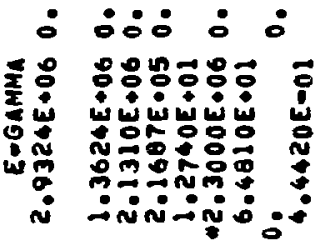

崖:

w是

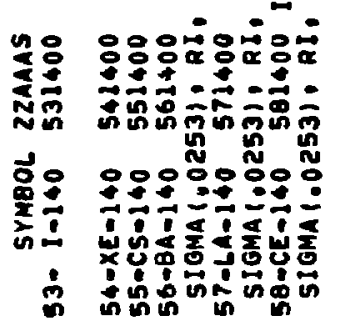
농융용용 山ش กN. 증요용 कू००:?

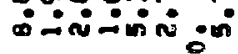

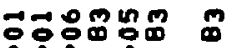
ज代

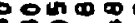
용요 $-+=$

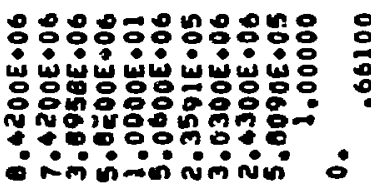

0090000000 $0008.00 \div 80:$

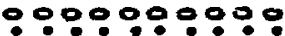

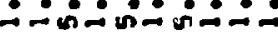

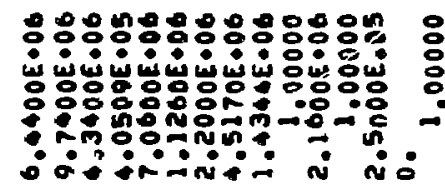

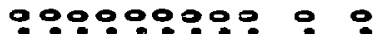
유융ㅇ :

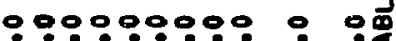

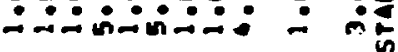

용융ㅇㅇㅇㅇㅇ -

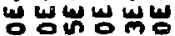

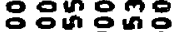

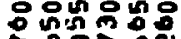
ํำ: Ón日

00000 $\therefore 0 \dot{0}: \dot{0}$ 웅ㅇㅇㅇ - nn- n-

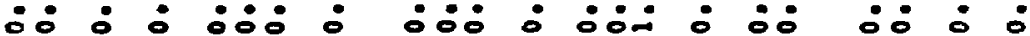
융 응 눙휴웅 is

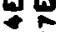
8 in

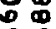
in

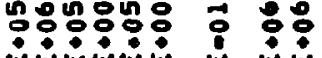
i.

:

$\div:$ i:

$\overrightarrow{0}:$ 岁岁 ni 용요 $\ddot{*}$

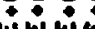
녕녕 웅웅

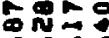
Din : ng요용 山岀山 No용 송요영 $\therefore \therefore$ in$$
\text { 政 }
$$

융유:

峞岗 음 \& 20 inis:

\section{$: 8:$ w w 웅

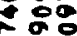 $\therefore$ i:} : 웅용 : 山w

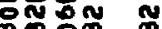
g. 的西二 = 屾酸向

o - o oooñ.

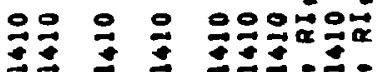
$\overrightarrow{\mathrm{N}} \overrightarrow{\mathrm{F}}$ in 두ㅇㅠㅜ웅 《ับ

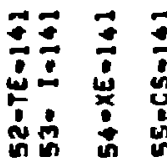
두응 $\div \div \div \div=$

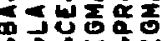
1. 훙유 山心 눙응 오ำ $\div=$

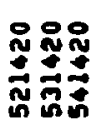
$N \times$ $\div \div \div$ แั ín: 命角药

\section{$:$}

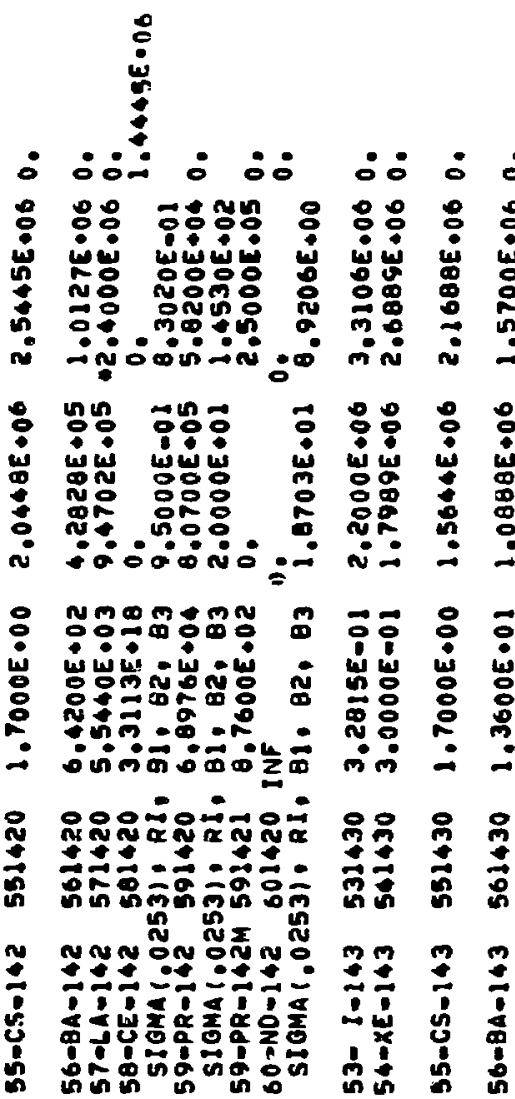




\begin{tabular}{|c|c|c|c|c|c|}
\hline 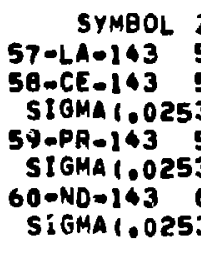 & $\begin{array}{l}\text { Z2AAAS } \\
571630 \\
581630 \\
5110 R 1 . \\
591630 \\
; 31 . R 1 . \\
6014301 \\
531 . R 1 .\end{array}$ & $\begin{array}{r}\text { HALFLIFE } \\
8,4000 E+02 \\
1,1880 E \cdot 05 \\
B 1.02, B 3 \\
1,1733 E+06 \\
B 1.82, B 3 \\
I N F \\
B 1, B 2, B 3\end{array}$ & $\begin{array}{r}E-B E T A \\
8.3128 E \bullet 05 \\
6.1913 E+05 \\
6.0000 E \bullet 00 \\
3.2392 E \bullet 05 \\
8.9000 E \bullet 01 \\
0.900 \\
3.2500 E \bullet 02\end{array}$ & 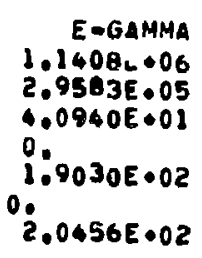 & $\begin{array}{l}0 . \text { EDAlPHa } \\
0 . \\
0 . \\
0 .\end{array}$ \\
\hline $\begin{array}{l}53-1-144 \\
54-x E=144 \\
55-C S-144\end{array}$ & $\begin{array}{l}531440 \\
541440 \\
551940\end{array}$ & $\begin{array}{l}1.3270 E-01 \\
1.0000 E \cdot 00 \\
1.0200 E \cdot 00\end{array}$ & $\begin{array}{l}3.0135 E+06 \\
1.2006 E+06 \\
2.3497 E+06\end{array}$ & $\begin{array}{l}1.2031 E \cdot 06 \\
2.0039 E=06 \\
3.0413 E=06\end{array}$ & $\begin{array}{l}0 . \\
0: \\
0:\end{array}$ \\
\hline $\begin{array}{l}56-8 A-164 \\
57-1 A-146 \\
58-C E-146\end{array}$ & $\begin{array}{l}561440 \\
571640 \\
581440\end{array}$ & $\begin{array}{l}1.1000 E \bullet 01 \\
6.0000 E \bullet 01 \\
2.4572 E \bullet 07\end{array}$ & $\begin{array}{l}6.4787 E+05 \\
1.5105 E+06 \\
0.2960 E+06\end{array}$ & $\begin{array}{l}1,0062 E<06 \\
1,9365 E+06 \\
2.8870 E+04\end{array}$ & $\begin{array}{l}0 .: \\
0 .\end{array}$ \\
\hline $\begin{array}{l}\text { SIGMA } 1.025 \\
\text { 59MPR-164 } \\
\text { 59mPR=164M }\end{array}$ & $\begin{array}{l}5310 R 1 . \\
591460 \\
591441\end{array}$ & $\begin{array}{l}81,02,03 \\
1.0360 E \cdot 03 \\
4.3200 E \cdot 02\end{array}$ & $\begin{array}{l}1.0000 E \cdot 00 \\
1.2628 E \bullet 06 \\
3.0000 E \cdot 02\end{array}$ & $\begin{array}{l}?, 0640 E \bullet 00 \\
3,1010 E \cdot 04 \\
5,0730 E * 04\end{array}$ & $0_{0 .}^{0 .}$ \\
\hline $\begin{array}{l}\text { 60-ND }-144 \\
\text { SIOMA } 1.025\end{array}$ & $\begin{array}{l}601440 \\
310 \mathrm{RI} .\end{array}$ & $\begin{array}{l}6,6226 E+22 \\
\theta i \\
0\end{array}$ & $6002 E \bullet 00$ & $\begin{array}{l}0 . \\
5.6153 E+00\end{array}$ & $72 E \cdot 06$ \\
\hline $\begin{array}{l}53-1-145 \\
54-x \in-145 \\
55-C S-145\end{array}$ & $\begin{array}{l}531+50 \\
541450 \\
551450\end{array}$ & $\begin{array}{l}1.0670 E-01 \\
9.0000 E=01 \\
5.6000 E-01\end{array}$ & $\begin{array}{l}2.4325 E \bullet 06 \\
1.9857 E \bullet 06 \\
1.6407 E \bullet 06\end{array}$ & $\begin{array}{l}3,7949 E \bullet 06 \\
3.0504 E \cdot 06 \\
2,3811 E+06\end{array}$ & $\begin{array}{l}0 . \\
0: \\
0 .:\end{array}$ \\
\hline $\begin{array}{l}56-8 A-145 \\
57-L A-145 \\
58-C E-145 \\
59-P A-145 \\
\text { 60-ND-145 } \\
\text { SPOMA 1.025 }\end{array}$ & $\begin{array}{l}561450 \\
571450 \\
581450 \\
591450 \\
601450 \\
31.11 .\end{array}$ & $\begin{array}{l}6,2000 E \cdot 00 \\
2,9000 E+01 \\
1,9800 E \cdot 02 \\
2,1520 E \bullet 04 \\
1 N F \\
B 1, B 2, B 3\end{array}$ & $\begin{array}{l}1.2866 E+06 \\
1.0582 E * 06 \\
6.2994 E+05 \\
7.0465 E+05 \\
5 . \\
4.1998 E * 01\end{array}$ & $\begin{array}{l}1.9210 E \cdot 06 \\
1.5196 E+06 \\
7.4890 E * 05 \\
1.3760 E+04 \\
0 . \\
2.2644 E .02\end{array}$ & $\begin{array}{l}0 . \\
0: \\
0: \\
0:\end{array}$ \\
\hline $\begin{array}{l}54=X E-146 \\
55-C 5-146\end{array}$ & $\begin{array}{l}541460 \\
551460\end{array}$ & $\begin{array}{l}9.3718 E-01 \\
1.9000 E=01\end{array}$ & $\begin{array}{l}.4492 E \cdot 06 \\
.4784 E \cdot 06\end{array}$ & $\begin{array}{l}2,5041 E \bullet 06 \\
3.3232 E \bullet 00\end{array}$ & 0. \\
\hline $\begin{array}{l}56-B A-146 \\
57-b A-146 \\
50-C E-146 \\
59-P R-146 \\
\text { 60-ND-146 } \\
\text { SIOMAI }\end{array}$ & $\begin{array}{l}561460 \\
571660 \\
581660 \\
591460 \\
601460 \\
31.41 .\end{array}$ & $\begin{array}{l}2.2000 E+00 \\
8.3000 E \bullet 00 \\
8,5200 E * 02 \\
1,4520 E+03 \\
\text { INF }\end{array}$ & $\begin{array}{l}7.2467 E \cdot 05 \\
1.7677 E \bullet 00 \\
2.426 \% E .05 \\
9.2790 E \cdot 05 \\
i .3996 E \cdot 00\end{array}$ & $\begin{array}{l}1.2159 E \bullet 06 \\
2.3575 E \bullet 06 \\
3.1430 E \bullet 05 \\
1.6349 E \bullet 06 \\
0,3,3004 E \cdot 00\end{array}$ & $\begin{array}{l}0 . \\
0: \\
0: \\
0: \\
0 .\end{array}$ \\
\hline $\begin{array}{l}54-K E-147 \\
55-C S-147 \\
56-B A-147\end{array}$ & $\begin{array}{l}5414 ? 0 \\
551470 \\
561470\end{array}$ & $\begin{array}{l}2.63 B 4 E-01 \\
5,5785 E-01 \\
2,2274 E .00\end{array}$ & $\begin{array}{l}2.1038 E \cdot 06 \\
1.9626 E \cdot 06 \\
1.0413 E .06\end{array}$ & $\begin{array}{l}3.5323 E \bullet 06 \\
2.9436 E \cdot 06 \\
2.2169 E .06\end{array}$ & $\begin{array}{l}0 . \\
0 . \\
0 .\end{array}$ \\
\hline
\end{tabular}

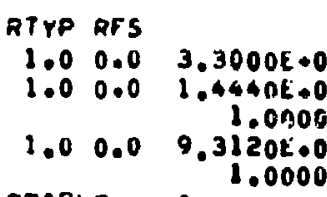

STABLE

$1.0 \quad 0.0 \quad 1.0230 E .07$

$1.00 .0 \quad 4.6700 E+06$

$1.00 .0 \quad 6.0500 E .06$

$5.00 .9 \quad 1.0054 E 006$

$1.00 .0 \quad 2.6900 E .06$

$1.0000 \quad 5.6000 E \cdot 06$

$1.00 .0 \quad 3.1550 E \cdot 05$

$1.01 .02 .5650 E .05$

$1.00 .0 \quad 2.99600 .06$

$1.00 .0 \quad 3.0556 \mathrm{E} \cdot 06$

$3.0 \quad 0.0 \quad 5.9000 E 004$

$4.00 .0 \quad 1.09000 .06$
T.00000

$1.0 \quad 0.0 \quad 8.66000 .06$

$1.0 \quad 0.0 \quad 7.1400 E .06$

$1.00 .06 .0700 E .06$

$5.0 \quad 0.0 \quad 2.2360 E \cdot 06$

$1.00 .0 \quad 4.95005+06$

$1.0 \quad 0.0 \quad 4.1500 E+06$

$1.0 \quad 0.0 \quad 2.4900 E 006$

$1.00 .0 \quad 1.0050 E .06$ STAOLE

1.00 .0

1.00 .0

5.00 .0

$1.0 n .0$

1.00 .0

1.00 .0 STABLE

.

1.00000

$5.5700 E \cdot 08$

8.5400E.06

2.0056E.06

2.9700 .06

$6.4500 E \cdot 06$

$1.0500 E+06$

$.0000 E .06$

0 .

$1.0 \quad 0.0 \quad 7.9000 E \cdot 06$

$1.0 \quad 0.0 \quad 6.9700 E \cdot 06$

$1.00 .0 \quad 5.440 C E .06$
BARNCWINA

$1.0000 F \cdot 00$

$1.0000 E_{000}$ 0.00001

$1.0000 E \cdot 00$

0 .

0.000 no

$0.070 n 0$

$1.0000 E-00$

$1.0000 E \bullet 00$

$9,5900 E-01$

$1.1000 E-0$ ?

$1.0000 E \bullet 00$

$1.0000 E 000$

$9.0000 E-01$

$1.2000 E-10$ ? $0.000 n a$

$1.0000 E+00$

5.0000E-04

$9.9950 E-01$

$.0000 E=01$

0.000 n

$1.0000=000$

$1.0000 E+00$

$9.5600 z=0$ I

404000 - va

$1.00000 \bullet 00$

$1.0000 E \cdot 00$

$1.0000 E=0 \mathrm{~B}$

$1.0000 E .00$

0.

0,00000

$1.0000 E .00$

$9.6100 E=0$

3.9ODOE-O2

$1.0000 E \cdot 00$

$1.0000 E \bullet 00$

$1.0000 E_{400}$

$1.0000 E 000$

0.

0.0 nona

$1.00005 \cdot 0 n$

$1.0000 E \cdot 00$

$1.0000 E .00$
AEH NDK NSP MAT $\begin{array}{lll}1.61698 .02 & 1 & 0 \\ 1.61696 .02 & 601\end{array}$ 0.00000

$1.166 E \cdot 02$ 0.00040

$1.4168 k \cdot 02$
0.00000

11695

$0 \quad 0714$

$1.42: 3 \pm 002,0586$

$1.4270 E \cdot 02 \quad 1 \quad 0609$

$1.4269 E .02$ 2 0627

$1.4269 E .02 \quad 10647$

$\begin{array}{lll}1.426 \theta E+02 & 1 & 0 \\ 1.0268 E+02 & 2 & 262\end{array}$

2678

0.00000

$1.4268 E \bullet 02$
$1.4268 E \bullet 02$

1.42675 .02

12696

$2 \quad 2697$

ऽ. 00000

1.43705 .02

$1.4370 E \bullet 02$

$1.0366 E \cdot 02$

$1.0360 E \cdot 02$

$1.0367 \ell \cdot 02$

$1.4367 E .02$

$1.0367 E \cdot 0 z$

0.00000

$1.0069 E+02$

$1.4468 E * 02$

$1.6468 E \cdot 02$

$1.0467 E+02$

1.44605 .02

$1.6066 E .02$

$1,44665.002$

0.60000

0715

$1.0560 E+02$

$1.4568 \mathrm{O}+02$

$1.4567 E .02$
0987 $\begin{array}{lll}1 & 0 & 610 \\ & 0 & 620\end{array}$

a 648

$\begin{array}{lll}0 & 63\end{array}$

$26 \%$

2698

0716

0611

0 b20

0609

0664

2600

2699

$\begin{array}{ll}0 & 17\end{array}$

$0 \$ 12$

$0 \quad 630$

0650 
SYMBOL ZZAAAS HALFLIFE 57-LA-147 571470 1.0000E•OL 58-CE-147 581470 7.0000E•01 59-PR-147 $391470 \quad 7.2000 E 002$ 60-ND-147 $601470 \quad 9.4954 E \cdot 05$ SIGMA (.0253), RI, BI, 82, B3 $61-P M-147 \quad 611470 \quad 0.2776 E+07$ SIGMA (.0253), RI, B1, 82, B3 62-SM-147 621470 3.3744E-18 SIOMA $(.0253), R J$. BI, B2, B3

55-CS-148 551480 2.0163E-01 56-8A-168 561480 5.9009E+00 57-LA-148 $\quad 371480 \quad 1.3000 E+00$ 5B-CE-148 SB14BD $4.3000 E .01$ $59+P R-148$
601480 I.
$60-140$
601480 INF

SIGMA $(.0253), R J, B 1,82,83$ $61-P M-148 \quad 611480 \quad 4.6397 E+05$ SIGMA $(.0253), A I, B i, 82, B 3$ $61 \cap P M-14 \theta M$ 61j481 3.5683E.06

SIOMA $(.0253), R !, 01,82,83$ $62-S M-148 \quad 621480 \quad 2,5229 E \cdot 23$ SIGMA (.0253) RI, BI, B2, B3

55-C5-149 551490 $2.7822 E-01$ $56+B A-149 \quad 561490 \quad 9,1747 E-01$ $57-14-149 \quad 571490 \quad 2,8638 E .00$ 58-CE-149 581490 1,0000E 00 59.PR-149 591490 1.3800E.02 $60-N O-149 \quad 601490 \quad 6.2280 E .03$ $61-P M-149 \quad 611490 \quad 1.9116 E \cdot 05$ SIGMA (0253), RI, BI, B2, B3 62-SM-169 $021490 \quad 3,1536 E-23$ SI GMA (.0253): RI, BI, B2, B3

55-CS-150 551500 $1.2437 E-01$ $56-8 A-150 \quad 561500 \quad 1.7975 E+00$ $57-L A-130$ 571500 6.4850E-01 $\begin{array}{lll}57-C A-150 & 571500 & 6.4050 E-01 \\ 5 B-C E-150 & 581500 & 1.0000 E\end{array}$ $\begin{array}{lll}58-C E-150 & 581500 & 1,0000 E+00 \\ 59-P R-150 & 591500 & 1.2400 E * 01\end{array}$ 61) N NO-150 601500 INF

SIGMA (0253)

i. 20000.00 i.6845E.01 0 61.PM-

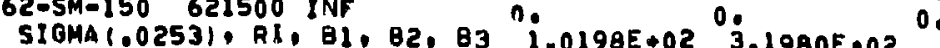

1.726450060 B.5132E•05 $7.4799 E+05$ $2.1169 E+05$ $4.9000 E_{\circ} 01$ $6.3000 E \cdot 04$ $1.8190 E \bullet 02$ 0 .

$6.4000 E \cdot 01$

2.7237E.06 $9.5593 E \circ 0^{\circ}$ $1.9341 E+06$ $3.6692 E+05$

$2.5000 \mathrm{E} 00$

$7.4430 E \cdot 05$

$2,0000 E \cdot 03$

$1.0616 E \bullet 04$

2:7000E•00

$1.6421 E+06$

$1.399 B E+06$

$9.8953 E+05$

$1.1578 E+06$
$+.7439 E \bullet 05$

$3.7658 E+05$

$1.0000 E .03$

0 :

$4.1190 E .04$

$2.9189 E 006$

$1.2180 E \circ 06$

$2.1426 E+06$

$5.5188 E+05$

$1.2714 E .06 \%$ 0.2009E+05 0. $1.1870 E+050$. $6.4780 E \triangle 02$

$1.0000 E+020$. $2.2832 E \cdot 03$ 0. 2.3

$7.4812 E .02$

3.8427E•06 0 .

$1.6636 E \bullet 060$.

2.6665E॰06 0 .

$6.1958 E+050$.

OE $\bullet 050$

2.0062E.01

$6.3037 E \cdot 050$.

$4.0010 E \cdot 04$

$2.0094 E .060$.

$3.6078 E \cdot 03$

$3.4825 E .060$.

$2.6658 E .060$.

$2.1822 E .06$ O.

$1.5240 \mathrm{E} \cdot 06 \mathrm{O}$.

$2.5126 E_{005} 0$.

$3.3675 E .050$.

$1.4230 E \cdot 040$.

Q.0120E.02

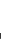

E-ALPHA RTYP RTS

$1.4738 E \cdot 05$

$2.2388 E \cdot 06$

$2.3298 E+06 \quad .0 .0 .0$

1.00 .0

$1.0 \quad 0.0 \quad 2.7000 E+06$

$1.0 \quad 0.0 \quad 0.9450 E+05$

1.00000

$1.0 \quad 0.0 \quad 2.2450 E+05$ .53000

1400000

$2,7420 E \bullet 012,0184 E \cdot 06$

$1.9076 E \cdot 06$

$1.0 \quad 0.0 \quad 9.2900 E \bullet 06$

$1.0 \quad 0.0 \quad 3.870$ E $० 06$

$1.00 .0 \quad 6.9300 E \bullet 06$

$1.00 .0 \quad 1.5300 E+06$

$1.00 .00 .8600 E+06$

STABLE

0.

$1.00 .0 \quad 1.00000$

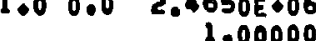

$1.00 .0 \quad 2.6020 E .06$

$3.00 .0 \quad 1.37205 .05$

1.00000

$4.00 .0 \quad 2.0009 E 000$

$1.00 .0 \quad 7.9600 E+06$

$1.00 .0 \quad 6.2000 E \cdot 05$

$1.00 .0 \quad 5.3600 E+06$

$1.0 \quad 0.0 \quad 3.9300 E \cdot 06$

$1.00 .03 .0000 E .06$

$1.00 .0 \quad 1.6800 E \circ 0 \mathrm{~L}$

$1.0 \quad 0.0 \quad 1.0724 E \bullet 06$ 1.00000

4.00 .01 .09495 .06

$1.0000 n$

$1.0 \quad 0.0 \quad 1.0180 E \bullet 07$

$1.0 \quad 0.0 \quad \$ .8700 E+06$

$1.0 \quad 0.0 \quad 7.6800 E \cdot 06$

$1.00 .02 .3600 E+06$

$1.0 \quad 0.0 \quad 5.0900 E+06$

STABLE

$1.00 .0 \quad 1.00000$

STABLE

1.00000
$3.4300 E * 06$

1.00000

$1.00 .07 .0800 E \cdot 06$
BRANCHING

$1.0000 E \bullet 00$

$1.0000 E .00$

. OOOOE 400

0000E•On

0,000 no

$1.0000 E \bullet 00$ - 70 To

$1.0000 E \cdot 00$

$0,000 \cap 0$

$1.0000 E \bullet 00$

$1.0000 E+00$

$1.0000 E<00$

$1.0000 E .00$

$1.0000 E \bullet 00$

0

0.00000

$1.0000 E+00$

0.000 ro

$9.0000 E-01$

$6.0000 E-02$ 0,00000

$1.0000 E+00$
0.00000

$1.0000 E+00$

$1.0000 E+00$

$1.0000 E+00$

$1.0000 E \bullet 00$

$1.0000 E+00$

$1.0000 E .00$

$1.0000 E \bullet 00$ 0.00000

$1.0000 E+00$

0.00000

$1.0000 E \bullet 00$

$1.0000 E+00$

$1.0000 E+00$

1.00005 .00

$1.0000 E \bullet 00$

0.

$0.000 n a$

$1.0000 E+00$

0.00000

AHA NDK NSP MAT

$1.4566 E .02 \quad 10665$ $1 . \$ 566 E+02$ I 0601

4566E+02 12700

$.4565 E+02 \quad 12718$

0.00000

$.4565 E+02$ 0.00000

12733

$.4565 E+02$

0.00060

$1.46676+02$

$1.4666 E \bullet 02$

$1.4606 E \bullet 02$

$1.4665 E+02$

$1.4665 E+02$

$1.4685 F .02$ 0.00000

$1.4685 E+02$

0.00000

$1.4665 E+02$

0.00000

$1.46646+02$

0.00000

$1.6767 E+02$

$1 . \$ 766 E+02$

$1.4765 E .02$

$1.4765 E+02$

$1.4764 E+02$

$1.4764 E \cdot 02$

$1.47646 \cdot 02$ 0.00000

$1.4764 E \bullet 02$

0.00000

1.4466Ë・02

1. $465 E .02$

1.4064E.02

$1.4064 E .02$

$1,4063 E+02$

0.00000

$1.4663 E-02$

0.00000

$1,0000 E+00$

$1.4965 E+02$
10753

10631

0 651

0466

68

- 719

13796

$2 \quad 2735$

10754

$0 \quad 032$

- 52

0667

6483

702

2736

- 755

o 633

0653

0668

- 64

0703

l 0737 

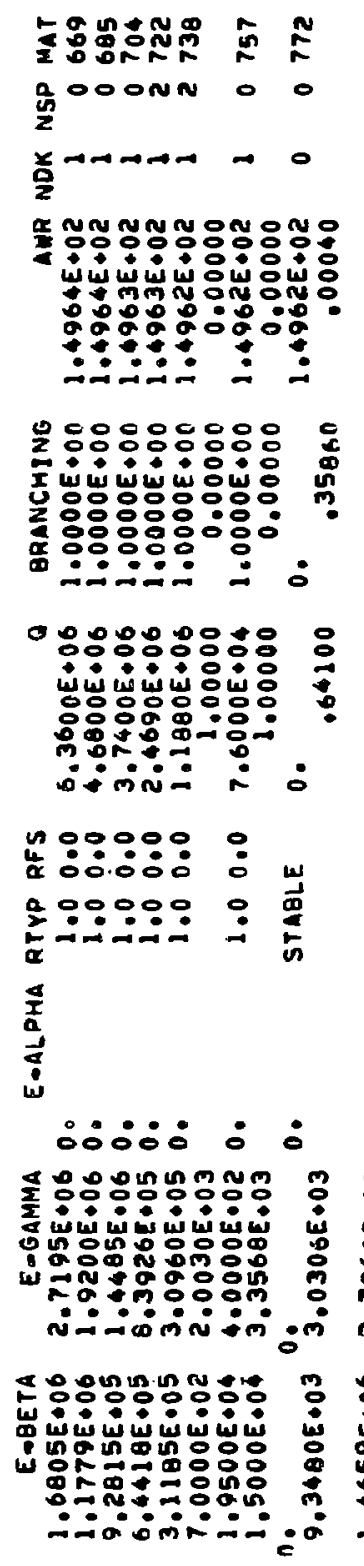

훙ㅇㅇㅇㅇㅇㅇㅛ 1

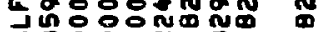

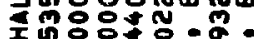

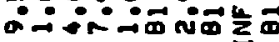

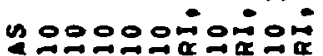

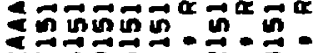

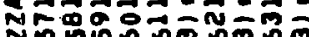

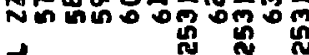

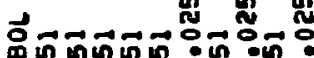

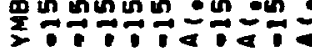

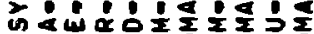

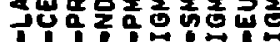

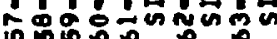

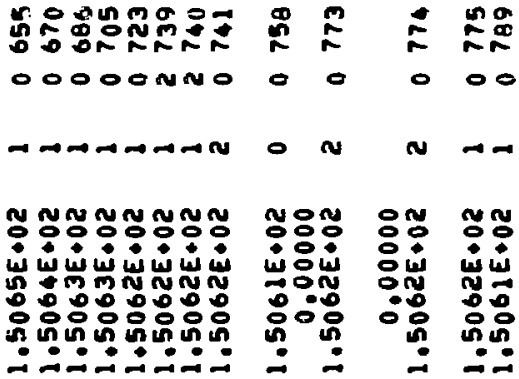

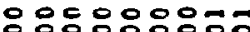
$: \div \circ 90.89:$ ساه 영ㅇㅇㅇㅇㅇㅇㅇㅇㅇㅇㅇㅇ 응ㅇㅇㅇㅇㅇㅇㅇㅇㅇㅇ

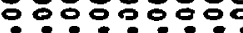

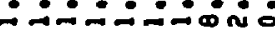

옹요용요 t. t 山岁峈岇骖峈 응응ㅇㅇㅇㅇㅇㅇㅇㅇ 옹ㅇํ웅ㅇㅇㅇㅇㅇㅇㅇ은 -

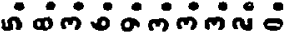

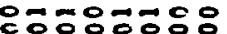

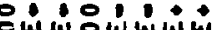
등녕녕닝 응영영응응 $\circ 8008008$ 는?

옹웅ㅇㅇㅇㅇㅇㅇㅇㅇ 운온

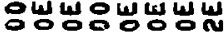
웅응ㅇㅇㅇ - 언 边 000000000 영ㅇㅇㅇㄴ $000000000 \frac{1}{10}$ $\because \therefore \div \div \div: \div 9=$

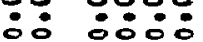
웅ㅇㅇㅇㅇㅇ in

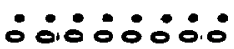

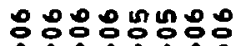

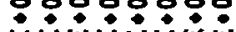

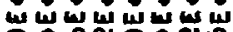

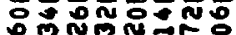

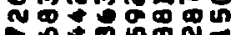
คำ แ⿻

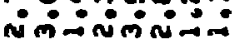
온ํำ

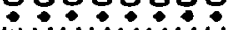
山嵌出的 ต

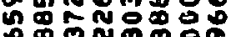

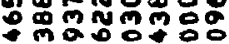
iñ⿻i一i 무워N 융ㅇㅇㅇㅇㅇㅇㅇㅇ

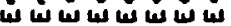

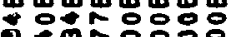

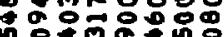
ñ. $\because m-\infty \dot{0}:=$

웅ㅇㅇㅇㅇㅇㅁㄸㅆ in w

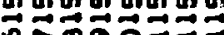

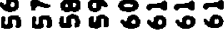

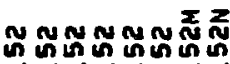
$\rightarrow \div 3 \div \div \div \div$

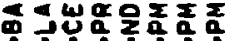
등유우웅
- $:$ :

영응 옹영

wi

ON

更

80 - in r.?

yon m

웅요

ज岾

ㅇ 8 \%

氠

은 ce

mo

s.

ำ

- o

$=\frac{0}{2}$

웅요

40 in

ติตี

a

นับ

$\because=\frac{1}{2}$

홍흔

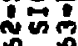
:0 옹쭈 - w 똥ㅇㅇㅇㅇㅇ 웅 :-

로 N $-\frac{6}{8}$ 총 $-2$

\section{要}

is

m: ติธ 预品 $\rightarrow ?$ 눙 ถ⿻ำ

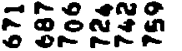

$\stackrel{2}{R}$

OOOONN

, 0

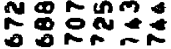

000000

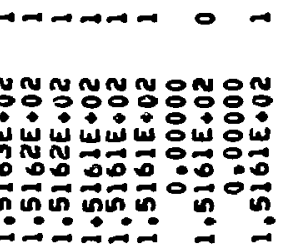

$\rightarrow \infty \rightarrow \infty$

บ $\mathbf{N}$

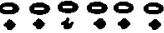
un wh ก $\rightarrow 00$ ำำำำ in in in in $\therefore \therefore \div \div \div$

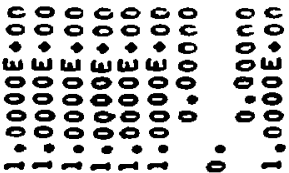

응동영웅후웅

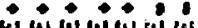
U山w 응용용용ㅇㅇ

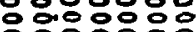
응ㅇㅇㅇㅡ.

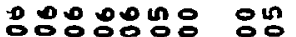

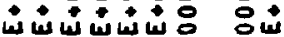

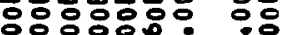
옹오웅유: 的公品。 in -1.

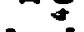

000000

영:유

$$
\text { 感 }
$$

붕요웅 $+4+t$

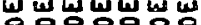
웅웅ㅇㅇㅇㅇㅇ 웅옹ㅇㅇ용 nnaranen 0ं

웅ㅇㅇㅇㅛ $\because \div \div \div \div \div$

0000000 ட்:ட்:்

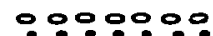
$\because \div \div \div \div 0$

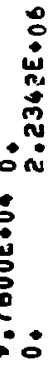

ப்0்ச0 : 웅ㅇㅇㅇㅇㅇㅇ m웅

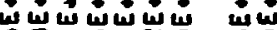

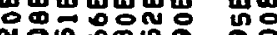

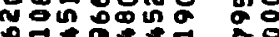
영 minan ㅇㅇㅇ영ํㅇ용

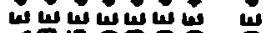

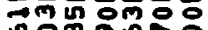
응ㅇㅇㅇㅛ

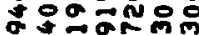
$\because \because \therefore 0$ im

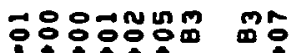
山幽的 - - -

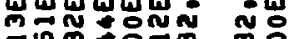

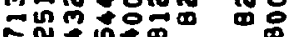

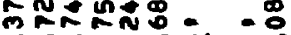

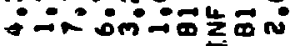

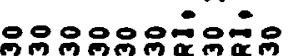

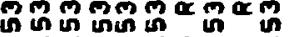
$\overrightarrow{2} \overrightarrow{0} \overrightarrow{0} \overrightarrow{0} \overrightarrow{0} \vec{N}=\vec{D}=\overrightarrow{0}$ 13

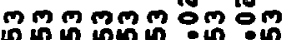
แn un un

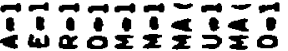

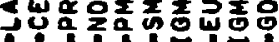

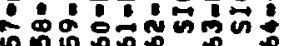

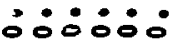
웅요

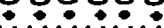
u ต - ํํㅇㅛㅛ No.0\%

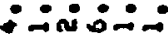

웅요봉요

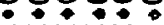

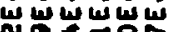
No눙

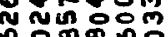
:요용 ann-

봉요 .

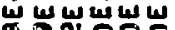
옹요

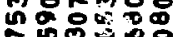
$\therefore \dot{0} \div 0 \div$ :

$00000 \%$

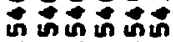

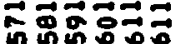

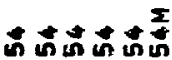
ำ:군

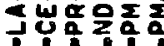
영우웅 


\begin{tabular}{|c|c|c|c|c|}
\hline 둘 & $\AA$ & & 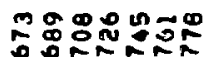 & \\
\hline$\frac{a}{2} 0$ & 0 & 0 & 0000000 & 0 \\
\hline 我o & $\rightarrow$ & 0 & 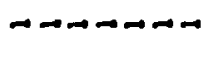 & 0 \\
\hline
\end{tabular}

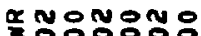

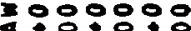
4영영ㅇㅇㅇ 녕영녕영영 D.0 0.0

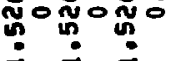

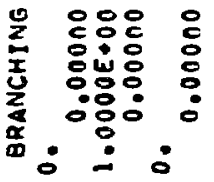

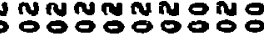

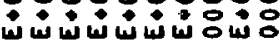

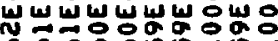

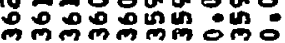

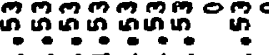

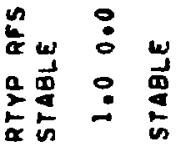

高 $\frac{1}{4}$

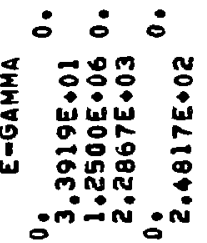

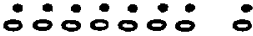

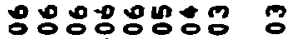
in.

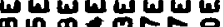
w n

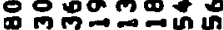
றீ inis:-

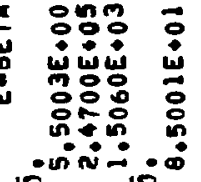

w $m m m$

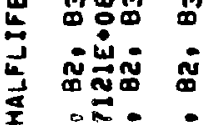

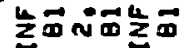

웅웅 in $\leq \dddot{n}-9-2$.

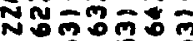
+ N N

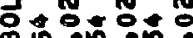

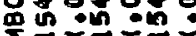

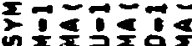

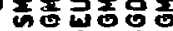

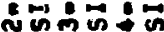
งูn

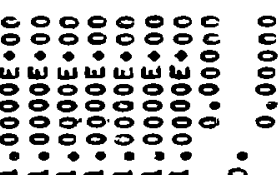

웅영영

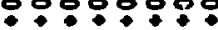

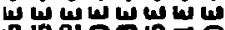

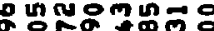

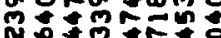

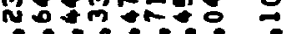

i $\rightarrow 0^{\circ}$ mín: $=0$

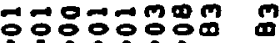

.

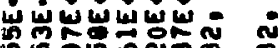

n

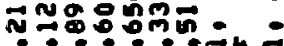

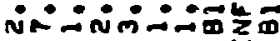

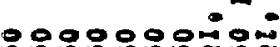

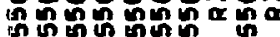

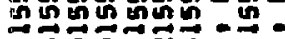

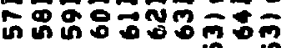
帘

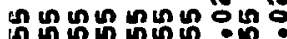

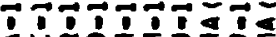

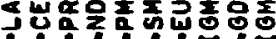

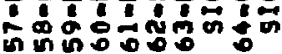

oga ona goNRTR

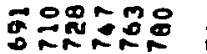
$=29500$ $\underline{2}$ 00000n o

000000

000000

o

ง $\operatorname{sen} 20$ - 9000900

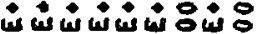
19ooj=95

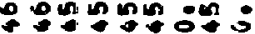

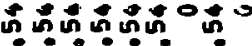

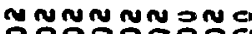
응ㅇㅇ응

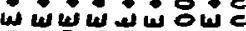

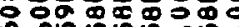
in in in in in on in in in in 0 in
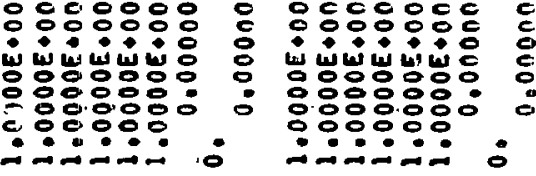

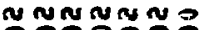

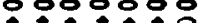

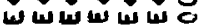
- 0 \% in in in un in:

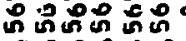
$\therefore \div$

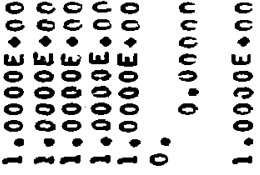

00040

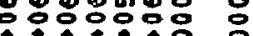

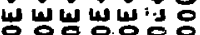
8 웅응용 융ㅇㅇㅇㅇㅛ in $00 \%$ जिnín:

0000000

00900

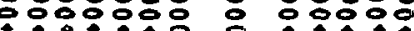

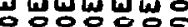
웅ㅇㅇㅇㅇ :

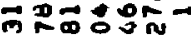
$\because \dot{0}: 0 \div a$

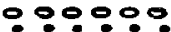

000000 웅ㅇㅇㅇ $\rightarrow \rightarrow \rightarrow \dot{-1}$

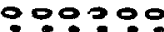

000050

웅ㅇㅇㅇㅇㅛ

$\rightarrow \rightarrow \rightarrow-\rightarrow$

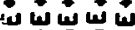

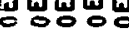

웅ㅇㅇㅇ

MNU

किं किं

00090

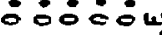

00000 T

.$: . .-\frac{8}{6}$

-

웅ㅇㅇㅇㅇㅇㅇ

넌

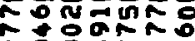

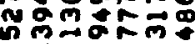

in:

-

ogngun

웅요.

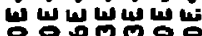

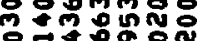

궁용요

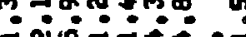

무요 m

웅ㅇㅇㅇ

山山山山山。

충응요 엉

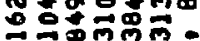

$\because \because \div$ -

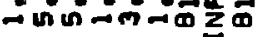

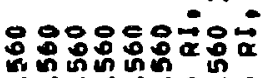

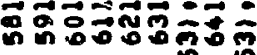

है

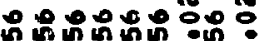

구무둔

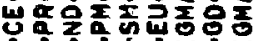

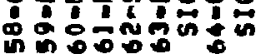

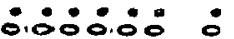

융ㅇㅇㅇㅇㅇ용 山山س P NARER N NAOAR:

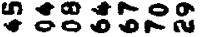
imici:- :

099 ํำ 웅ㅇㅇ

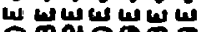
om - un

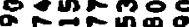
$\because \therefore \because-\div$ un

-10mNam

웅ㅇㅇㅇํํ

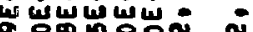

- 영ํㅇㅇㅝ

두요용

m 우웅우ㅇㅜㅜ

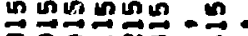

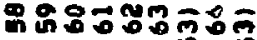
n máñ Oñ $\because \div \div \div \div \div \div=$

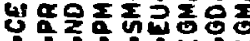

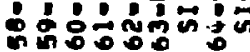

형ㅇㅇ :

웅융ㅇㅇ 웅

山 山ا

$\Rightarrow$ 궁요 $\mathrm{m}$



cưm m

m-iv:-

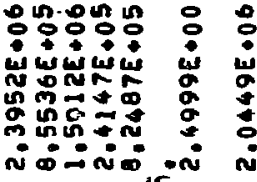

영웅 m

山以岀山

용요

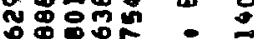

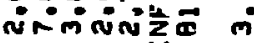

융요요요 논ํㅕㄴ

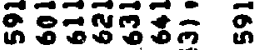

0 ก

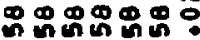
문무둥

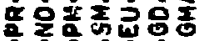
$20 \div 05$

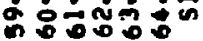

$+$ 


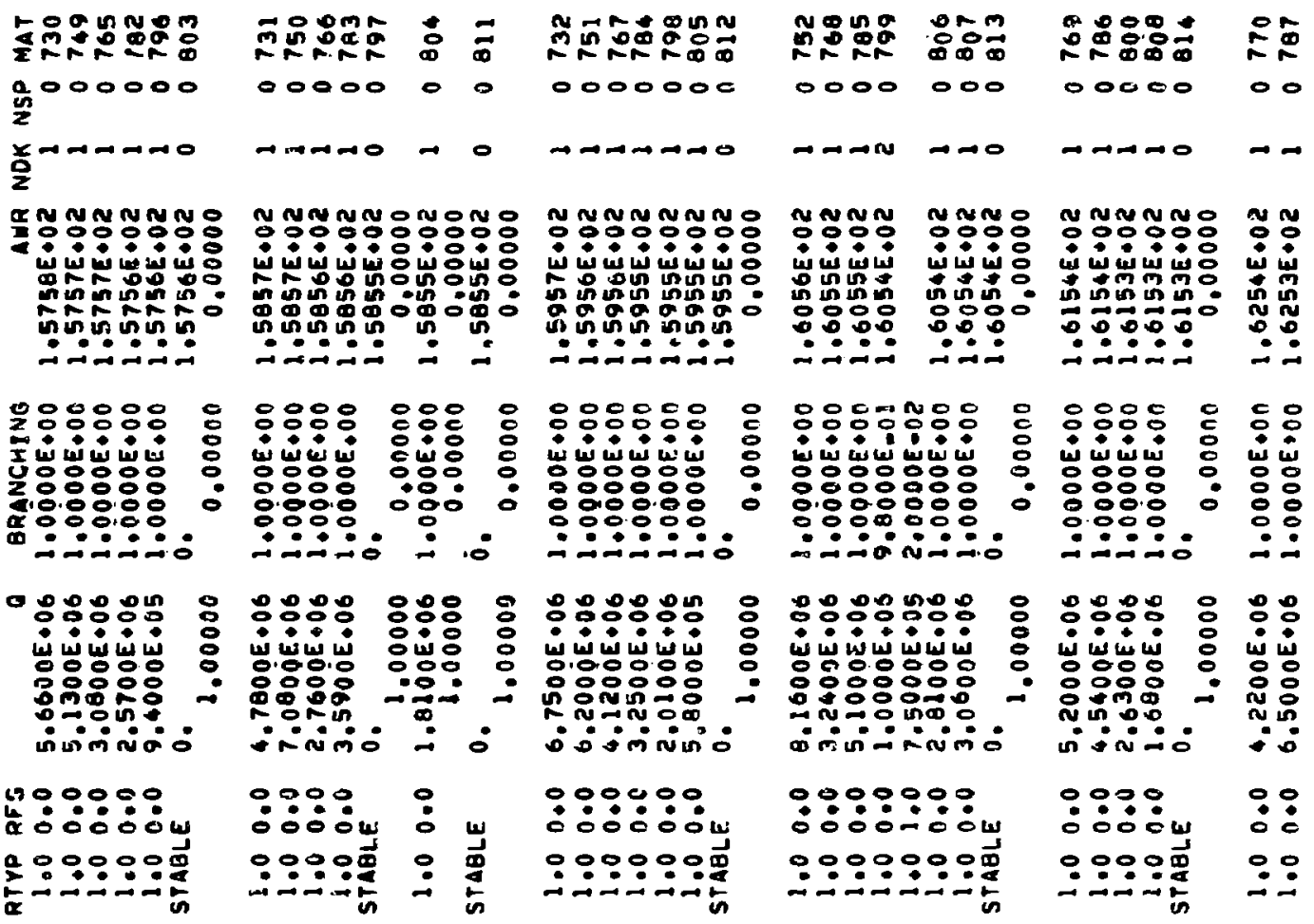

ํ.

பீ口த:

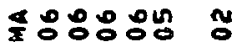
준유:

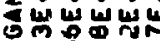

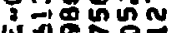

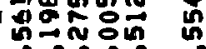
niñ-in:-

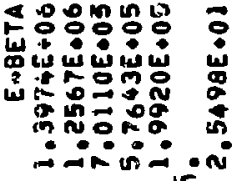
능용붕휴 1. 促 दํำ 또유ํำ

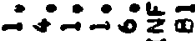

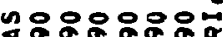

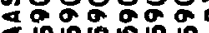
응문 N.6.0.00 하요요웅

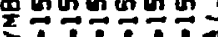

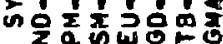
$101=$ ㅇํㅇํำ
ட்0்:- : : 융융ㅇㅇㅇㅇㅇㅇㅇ

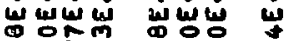

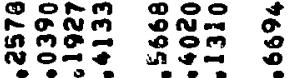

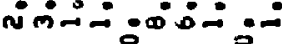

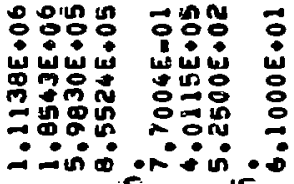

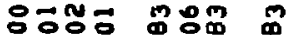
它它 ำกำ ஸ⿻一𠃋十

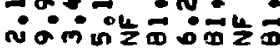

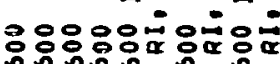
00000.00

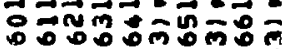
in $88880 \% 0 \%$

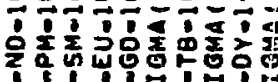

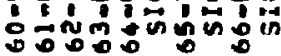

$\therefore 00400^{\circ}$

ํํㅇํำ

-

w w w

บ

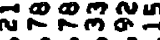

किंনnn:-

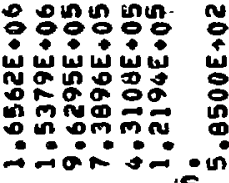

두:-in

유응뮤 山嵌㟧岩

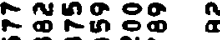

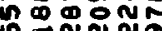

?Nำ

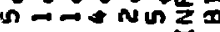

웅으우으으우

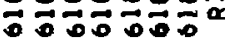

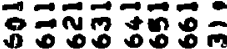
$\overrightarrow{0} \overrightarrow{0} \overrightarrow{0} \overrightarrow{0} \overrightarrow{0} \overrightarrow{0}$ 굴울

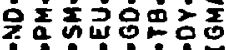

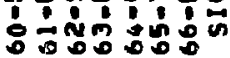

¿0:0

웅요

(1)

岁岕嵌

용

2:.8

$: \because \div:$

in

ํํㅇํํㅇ

웅유

思点司

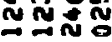

iñn

$\vec{B}$ Nㅗㅇ 유유.

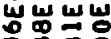
思罾: ․ํㅇ응 inis:

오옹ㅇ ดัด तิ มกับ กิํํㅇํㅇ I뭉 플용 ํํำ 요용

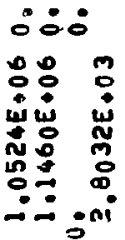

$\therefore \therefore 000$

$\therefore:$

ํํㅇ

융ㅇㅇ

山以山w w

N贻

호용

N

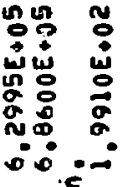

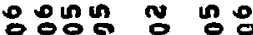

:ㅇ: : :

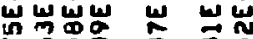

용요

ก:u n.

(n)

융 ๑

岁岁 N

通

$\$$

$+\infty \frac{4}{2}$

욱우

풍뭉

जिज़:

०००\%

บ No

บูบ:

구일

뼌중

ถำ

我占品

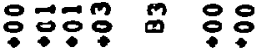

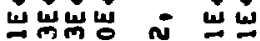

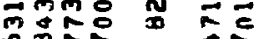

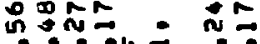

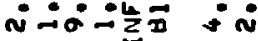

웅유물

00000

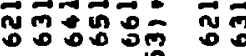

:

m.mm

:

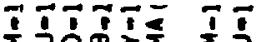

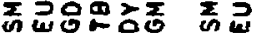

남은

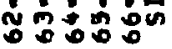




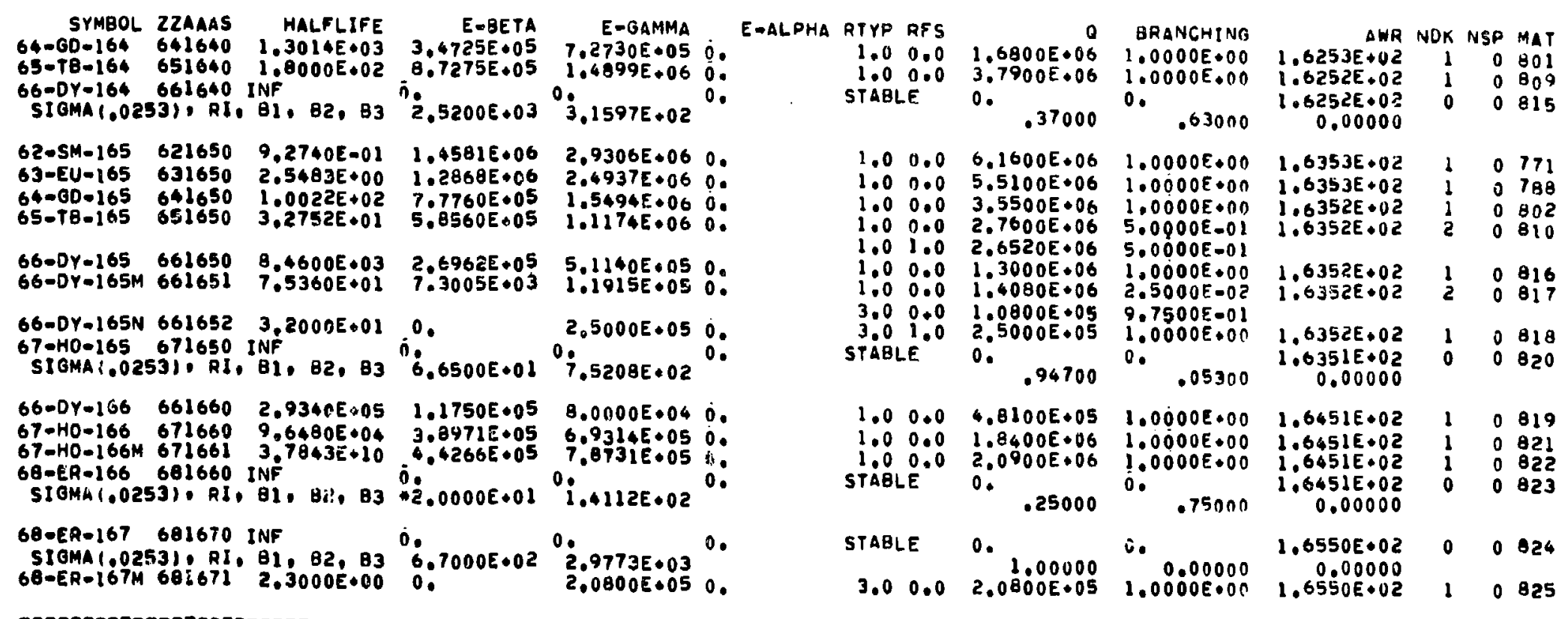

*Changed from ENDF/B-IV.

See Table v.

Recent investigation indicates that all ${ }^{98} \mathrm{zr}$ decays o the $2.8 \mathrm{~s}$ state of ${ }^{98} \mathrm{Nb}$; chinge RrS to 0.0 . 